\title{
Stratified-algebraic vector bundles
}

\author{
Wojciech Kucharz and Krzysztof Kurdyka
}

\begin{abstract}
We investigate stratified-algebraic vector bundles on a real algebraic variety $X$. A stratification of $X$ is a finite collection of pairwise disjoint, Zariski locally closed subvarieties whose union is $X$. A topological vector bundle $\xi$ on $X$ is called a stratified-algebraic vector bundle if, roughly speaking, there exists a stratification $\mathcal{S}$ of $X$ such that the restriction of $\xi$ to each stratum $S$ in $\mathcal{S}$ is an algebraic vector bundle on $S$. In particular, every algebraic vector bundle on $X$ is stratified-algebraic. It turns out that stratified-algebraic vector bundles have many surprising properties, which distinguish them from algebraic and topological vector bundles.
\end{abstract}

Key words. Real algebraic variety, stratification, stratified-algebraic vector bundle, stratifiedregular map.

Mathematics subject classification (2010). 14P25, 14P99, 14F25, $19 \mathrm{~A} 49$.

\section{Contents}

1 Introduction and main results 2

2 Stratified-regular maps 6

3 Basic properties of stratified-algebraic vector bundles 10

4 Approximation by stratified-regular maps 17

5 Topological versus stratified-algebraic vector bundles 23

6 Blowups and vector bundles 25

7 Stratified-algebraic cohomology classes 32

8 Stratified-C-algebraic cohomology classes 37

References

The first author was partially supported by NCN (Poland) grant 2011/01/B/ST1/01289.

The second author was partially supported by ANR (France) grant STAAVF. 


\section{Introduction and main results}

In real algebraic geometry, the role of algebraic, semi-algebraic and Nash vector bundles is firmly established. Vector bundles of a new type, called stratified-algebraic vector bundles, are introduced and investigated in this paper. Stratified-algebraic vector bundles form an intermediate category between algebraic and semi-algebraic vector bundles. They have many desirable features of algebraic vector bundles, but are more flexible. Some of their properties and applications are quite unexpected.

For background material on real algebraic geometry we refer to [9]. The term real algebraic variety designates a locally ringed space isomorphic to an algebraic subset of $\mathbb{R}^{N}$, for some $N$, endowed with the Zariski topology and the sheaf of real-valued regular functions (such an object is called an affine real algebraic variety in [9]). The class of real algebraic varieties is identical with the class of quasi-projective real varieties, cf. [9, Proposition 3.2.10, Theorem 3.4.4]. Morphisms of real algebraic varieties are called regular maps. Each real algebraic variety carries also the Euclidean topology, which is induced by the usual metric on $\mathbb{R}$. Unless explicitly stated otherwise, all topological notions relating to real algebraic varieties refer to the Euclidean topology.

Let $X$ be a real algebraic variety. By a stratification of $X$ we mean a finite collection $\mathcal{X}$ of pairwise disjoint, Zariski locally closed subvarieties whose union is $X$. Each subvariety in $\mathcal{X}$ is called a stratum of $\mathcal{X}$; a stratum can be empty. The stratification $\mathcal{X}$ is said to be nonsingular if each stratum in it is a nonsingular subvariety. A stratification $\mathcal{X}^{\prime}$ of $X$ is said to be a refinement of $\mathcal{X}$ if each stratum of $\mathcal{X}^{\prime}$ is contained in some stratum of $\mathcal{X}$. There is a nonsingular stratification of $X$ which is a refinement of $\mathcal{X}$. If $\mathcal{X}_{1}$ and $\mathcal{X}_{2}$ are stratifications of $X$, then the collection $\left\{S_{1} \cap S_{2} \mid S_{1} \in \mathcal{X}_{1}, S_{2} \in \mathcal{X}_{2}\right\}$ is a stratification of $X$ that is a refinement of $\mathcal{X}_{i}$ for $i=1,2$. These facts will be frequently tacitly used. The stratification $\{X\}$ of $X$, consisting of only one stratum, is said to be trivial.

Let $\mathbb{F}$ stand for $\mathbb{R}, \mathbb{C}$ or $\mathbb{H}$ (the quaternions). All $\mathbb{F}$-vector spaces will be left $\mathbb{F}$-vector spaces. When convenient, $\mathbb{F}$ will be identified with $\mathbb{R}^{d(\mathbb{F})}$, where $d(\mathbb{F})=\operatorname{dim}_{\mathbb{R}} \mathbb{F}$.

For any topological $\mathbb{F}$-vector bundle $\xi$ on $X$, denote by $E(\xi)$ its total space and by $\pi(\xi): E(\xi) \rightarrow X$ the bundle projection. The fiber of $\xi$ over a point $x$ in $X$ is $E(\xi)_{x}:=\pi(\xi)^{-1}(x)$. Denote by $\varepsilon_{X}^{n}(\mathbb{F})$ the standard trivial $\mathbb{F}$-vector bundle on $X$ with total space $X \times \mathbb{F}^{n}$, where $X \times \mathbb{F}^{n}$ is regarded as a real algebraic variety. By an algebraic $\mathbb{F}$-vector bundle on $X$ we mean an algebraic $\mathbb{F}$-vector subbundle of $\varepsilon_{X}^{n}(\mathbb{F}$ ) for some $n$ (cf. [9, Chapters 12 and 13] for various characterizations of algebraic $\mathbb{F}$-vector bundles). In particular, if $\xi$ is an algebraic $\mathbb{F}$-vector subbundle of $\varepsilon_{X}^{n}(\mathbb{F})$, then $E(\xi)$ is a Zariski closed subvariety of $X \times \mathbb{F}^{n}, \pi(\xi)$ is the restriction of the canonical projection $X \times \mathbb{F}^{n} \rightarrow X$, and $E(\xi)_{x}$ is an $\mathbb{F}$-vector subspace of $\{x\} \times \mathbb{F}^{n}$ for each point $x$ in $X$.

Given a stratification $\mathcal{X}$ of $X$, we now introduce the crucial notion for this paper.

Definition 1.1. An $\mathcal{X}$-algebraic $\mathbb{F}$-vector bundle on $X$ is a topological $\mathbb{F}$-vector subbundle $\xi$ of $\varepsilon_{X}^{n}(\mathbb{F})$, for some $n$, such that the restriction $\left.\xi\right|_{S}$ of $\xi$ to each stratum $S$ of $\mathcal{X}$ is an algebraic $\mathbb{F}$-vector subbundle of $\varepsilon_{S}^{n}(\mathbb{F})$. If $\xi$ and $\eta$ are $\mathcal{X}$-algebraic $\mathbb{F}$-vector bundles on $X$, an $\mathcal{X}$-algebraic morphism $\varphi: \xi \rightarrow \eta$ is a morphism of topological $\mathbb{F}$-vector bundles which induces a morphism of algebraic $\mathbb{F}$-vector bundles $\varphi_{S}:\left.\left.\xi\right|_{S} \rightarrow \eta\right|_{S}$ for each stratum $S$ in $\mathcal{X}$.

The conditions imposed on $\varphi$ mean that $\varphi: E(\xi) \rightarrow E(\eta)$ is a continuous map, $\pi(\xi)=\pi(\eta) \circ \varphi$, the restriction $\varphi_{x}: E(\xi)_{x} \rightarrow E(\eta)_{x}$ of $\varphi$ is an $\mathbb{F}$-linear transformation for each point $x$ in $X$, and the restriction $\varphi_{S}: \pi(\xi)^{-1}(S) \rightarrow \pi(\eta)^{-1}(S)$ of $\varphi$ is a regular map of real algebraic varieties for each stratum $S$ in $\mathcal{X}$. 
If $\xi$ is as in Definition 1.1, we also say that $\xi$ is an $\mathcal{X}$-algebraic $\mathbb{F}$-vector subbundle of $\varepsilon_{X}^{n}(\mathbb{F})$.

One readily checks that $\mathcal{X}$-algebraic $\mathbb{F}$-vector bundles on $X$ (together with $\mathcal{X}$-algebraic morphisms) form a category. An $\mathcal{X}$-algebraic morphism of $\mathcal{X}$-algebraic $\mathbb{F}$-vector bundles is an isomorphism if and only if it is a bijective map. In particular, the category of algebraic $\mathbb{F}$-vector bundles on $X$ coincides with the category of $\{X\}$-algebraic $\mathbb{F}$-vector bundles on $X$.

Definition 1.2. A stratified-algebraic $\mathbb{F}$-vector bundle on $X$ is an $\mathcal{S}$-algebraic $\mathbb{F}$-vector bundle for some stratification $\mathcal{S}$ of $X$. If $\xi$ and $\eta$ are stratified-algebraic $\mathbb{F}$-vector bundles on $X$, a stratified-algebraic morphism $\varphi: \xi \rightarrow \eta$ is an $\mathcal{S}$-algebraic morphism for some stratification $\mathcal{S}$ of $X$ such that both $\xi$ and $\eta$ are $\mathcal{S}$-algebraic $\mathbb{F}$-vector bundles.

A stratified-algebraic $\mathbb{F}$-vector subbundle of $\xi_{X}^{n}(\mathbb{F})$ is defined in an obvious way.

Stratified-algebraic $\mathbb{F}$-vector bundles on $X$ (together with stratified-algebraic morphisms) form a category. A stratified-algebraic morphism of stratified-algebraic $\mathbb{F}$-vector bundles is an isomorphism if and only if it is a bijective map.

Theory of $\mathcal{X}$-algebraic and stratified-algebraic $\mathbb{F}$-vector bundles is developed in the subsequent sections. In the present section, we only announce seven rather surprising results.

Denote by $\mathbb{S}^{d}$ the unit $d$-sphere,

$$
\mathbb{S}^{d}=\left\{\left(x_{0}, \ldots, x_{d}\right) \in \mathbb{R}^{d+1} \mid x_{0}^{2}+\cdots+x_{d}^{2}=1\right\} .
$$

Theorem 1.3. Let $X$ be a compact real algebraic variety homotopically equivalent to $\mathbb{S}^{d}$. Then each topological $\mathbb{F}$-vector bundle on $X$ is isomorphic to a stratified-algebraic $\mathbb{F}$-vector bundle.

Theorem 1.3 makes it possible to demonstrate an essential difference between algebraic and stratified-algebraic $\mathbb{F}$-vector bundles.

Example 1.4. It is well known that each topological $\mathbb{F}$-vector bundle on $\mathbb{S}^{d}$ is isomorphic to an algebraic $\mathbb{F}$-vector bundle, cf. [45, Theorem 11.1] and [9, Proposition 12.1.12; pp. 325, 326, 352]. This is no longer true if $\mathbb{S}^{d}$ is replaced by a nonsingular real algebraic variety diffeomorphic to $\mathbb{S}^{d}$. Indeed, for every positive integer $k$, there exists a nonsingular real algebraic variety $\Sigma^{4 k}$ that is diffeomorphic to $\mathbb{S}^{4 k}$ and each algebraic $\mathbb{F}$-vector bundle on $\Sigma^{4 k}$ is topologically stably trivial, cf. [8, Theorem 9.1]. However, on $\mathbb{S}^{4 k}$, and hence on $\Sigma^{4 k}$, there are topological $\mathbb{F}$-vector bundles that are not stably trivial, cf. [29]. On the other hand, according to Theorem 1.3 , each topological $\mathbb{F}$-vector bundle on $\Sigma^{4 k}$ is isomorphic to a stratified-algebraic $\mathbb{F}$-vector bundle.

Stratified-algebraic vector bundles on a compact real algebraic variety are in some sense stable with respect to stratifications. This is made precise in the following result.

Theorem 1.5. Any compact real algebraic variety $X$ admits a nonsingular stratification $\mathcal{S}$ such that each stratified-algebraic $\mathbb{F}$-vector bundle on $X$ is isomorphic (in the category of stratified-algebraic $\mathbb{F}$-vector bundles on $X)$ to an $\mathcal{S}$-algebraic $\mathbb{F}$-vector bundle.

It is remarkable that the stratification $\mathcal{S}$ in Theorem 1.5 is suitable for all stratifiedalgebraic $\mathbb{F}$-vector bundles on $X$. In general one cannot take as $\mathcal{S}$ the trivial stratification $\{X\}$ of $X$, even if $X$ is a "simple" nonsingular real algebraic variety, cf. Example 1.4 . 
A multiblowup of a real algebraic variety $X$ is a regular map $\pi: X^{\prime} \rightarrow X$ which is the composition of a finite number of blowups with nonsingular centers. If no additional restrictions on the centers of blowups are imposed, $\pi$ need not be a birational map (for example, a blowup of $X$ with center of dimension $\operatorname{dim} X$ is not a birational map).

Theorem 1.6. For any compact real algebraic variety $X$, there exists a birational multiblowup $\pi: X^{\prime} \rightarrow X$, with $X^{\prime}$ a nonsingular variety, such that for each stratified-algebraic $\mathbb{F}$-vector bundle $\xi$ on $X$, the pullback $\mathbb{F}$-vector bundle $\pi^{*} \xi$ on $X^{\prime}$ is isomorphic (in the category of stratified-algebraic $\mathbb{F}$-vector bundles on $\left.X^{\prime}\right)$ to an algebraic $\mathbb{F}$-vector bundle on $X^{\prime}$

The multiblowup $\pi: X^{\prime} \rightarrow X$ in Theorem 1.6 is chosen in a universal way, that is, it does not depend on $\xi$.

Any topological $\mathbb{F}$-vector bundle $\xi$ can be regarded as an $\mathbb{R}$-vector bundle, which is indicated by $\xi_{\mathbb{R}}$. If $\xi$ is $\mathcal{X}$-algebraic, then so is $\xi_{\mathbb{R}}$.

Theorem 1.7. Let $X$ be a compact real algebraic variety. A topological $\mathbb{F}$-vector bundle $\xi$ on $X$ is isomorphic to a stratified-algebraic $\mathbb{F}$-vector bundle if and only if the topological $\mathbb{R}$-vector bundle $\xi_{\mathbb{R}}$ is isomorphic to a stratified-algebraic $\mathbb{R}$-vector bundle.

This result is unexpected since it may happen that a topological $\mathbb{F}$-vector bundle $\xi$ (with $\mathbb{F}=\mathbb{C}$ or $\mathbb{F}=\mathbb{H}$ ) is not isomorphic to any algebraic $\mathbb{F}$-vector bundle, whereas the $\mathbb{R}$-vector bundle $\xi_{\mathbb{R}}$ is isomorphic to an algebraic $\mathbb{R}$-vector bundle, cf. the $\mathbb{C}$-line bundle $\lambda^{\mathbb{C}}$ in Example 1.11 .

Theorems 1.5, 1.6 and 1.7 are equivalent to certain approximation results, cf. Theorems $4.8,4.9$ and 6.6 .

It is possible to give, in some cases, a simple geometric criterion for a topological vector bundle to be isomorphic to a stratified-algebraic vector bundle.

Let $X$ be a compact nonsingular real algebraic variety. A smooth (of class $\mathcal{C}^{\infty}$ ) $\mathbb{F}$-vector bundle $\xi$ on $X$ is said to be adapted if there exists a smooth section $u: X \rightarrow \xi$ transverse to the zero section and such that its zero locus $Z(u):=\{x \in X \mid u(x)=0\}$, which is a compact smooth submanifold of $X$, is smoothly isotopic to a nonsingular Zariski locally closed subvariety $Z$ of $X$. In that case, $Z$ is a closed subset of $X$ in the Euclidean topology, but need not be Zariski closed. If $\operatorname{rank} \xi=\operatorname{dim} X$, then $\xi$ is adapted since the zero locus of any smooth section of $\xi$ that is transverse to the zero section is a finite set.

Theorem 1.8. Let $X$ be a compact nonsingular real algebraic variety. If a smooth $\mathbb{F}$-line bundle $\xi$ on $X$ is adapted, then it is topologically isomorphic to a stratified-algebraic $\mathbb{F}$-line bundle.

Theorem 1.8 with $\mathbb{F}=\mathbb{R}$ is not interesting since then a stronger result, asserting that $\xi$ is isomorphic to an algebraic $\mathbb{R}$-line bundle, is known, cf. [9, Theorem 12.4.6]. However, $\xi$ need not be isomorphic to any algebraic $\mathbb{F}$-line bundle for $\mathbb{F}=\mathbb{C}$ or $\mathbb{F}=\mathbb{H}$, cf. the $\mathbb{F}$-line bundle $\lambda^{\mathbb{F}}$ in Example 1.11

Assuming that $\mathbb{F}=\mathbb{R}$ or $\mathbb{F}=\mathbb{C}$, the $r$ th exterior power of any $\mathbb{F}$-vector bundle of rank $r$ is denoted by $\operatorname{det} \xi$. Thus, $\operatorname{det} \xi$ is an $\mathbb{F}$-line bundle.

Theorem 1.9. Let $X$ be a compact nonsingular real algebraic variety and let $\xi$ be a smooth $\mathbb{F}$-vector bundle of rank 2 on $X$, where $\mathbb{F}=\mathbb{R}$ or $\mathbb{F}=\mathbb{C}$. If both $\mathbb{F}$-vector bundles $\xi$ and $\operatorname{det} \xi$ are adapted, then $\xi$ is topologically isomorphic to a stratified-algebraic $\mathbb{F}$-vector bundle. 
It is not a serious restriction that the vector bundle $\xi$ in the last two theorems is smooth. In fact, any topological $\mathbb{F}$-vector bundle on a smooth manifold is topologically isomorphic to a smooth $\mathbb{F}$-vector bundle, cf. [28]. Actually, suitably refined versions of Theorems 1.8 and 1.9 hold true even if the variety $X$ is possibly singular, cf. Theorems 6.9 and 6.10 .

We select one more result for this section.

Theorem 1.10. Let $X=X_{1} \times \cdots \times X_{n}$, where each $X_{i}$ is a compact real algebraic variety homotopically equivalent to the unit $d_{i}$-sphere for $1 \leq i \leq n$. If $\mathbb{F}=\mathbb{C}$ or $\mathbb{F}=\mathbb{H}$, then each topological $\mathbb{F}$-vector bundle on $X$ is isomorphic to a stratified-algebraic $\mathbb{F}$-vector bundle. If $\xi$ is a topological $\mathbb{R}$-vector bundle on $X$, then the direct sum $\xi \oplus \xi$ is isomorphic to a stratified-algebraic $\mathbb{R}$-vector bundle.

We do not know if in Theorem 1.10 each topological $\mathbb{R}$-vector bundle on $X$ is isomorphic to a stratified-algebraic $\mathbb{R}$-vector bundle. This remains an open problem even for $\mathbb{R}$-vector bundles on the standard $n$-torus

$$
\mathbb{T}^{n}=\mathbb{S}^{1} \times \cdots \times \mathbb{S}^{1} \quad(\text { the } n \text {-fold product }) .
$$

It is worthwhile to contrast the results above with the behavior of algebraic $\mathbb{F}$-vector bundles on $\mathbb{T}^{n}$. As usual, the $k$ th Chern class of a $\mathbb{C}$-vector bundle $\xi$ will be denoted by $c_{k}(\xi)$. Any $\mathbb{F}$-vector bundle $\zeta$ can be regarded as a $\mathbb{K}$-vector bundle, denoted $\zeta_{\mathbb{K}}$, where $\mathbb{K} \subseteq \mathbb{F}$ and $\mathbb{K}$ stands for $\mathbb{R}, \mathbb{C}$ or $\mathbb{H}$. In particular, $\zeta_{\mathbb{F}}=\zeta$. If $\zeta$ is an $\mathbb{H}$-vector bundle, then $\zeta_{\mathbb{R}}=\left(\zeta_{\mathbb{C}}\right)_{\mathbb{R}}$.

Example 1.11. Every algebraic $\mathbb{C}$-vector bundle on $\mathbb{T}^{n}$ is algebraically stably trivial, cf. [11] or [9, Corollary 12.6.6]. In particular, every algebraic $\mathbb{C}$-line bundle on $\mathbb{T}^{n}$ is algebraically trivial. Consequently, for each algebraic $\mathbb{F}$-vector bundle $\zeta$ on $\mathbb{T}^{n}$, where $\mathbb{F}=\mathbb{C}$ or $\mathbb{F}=\mathbb{H}$, one has $c_{k}\left(\zeta_{\mathbb{C}}\right)=0$ for every $k \geq 1$. Furthermore, for each algebraic $\mathbb{R}$-vector bundle $\eta$ on $\mathbb{T}^{n}$, the direct sum $\eta \oplus \eta$ is algebraically stably trivial (it suffices to consider the complexification $\mathbb{C} \otimes \eta$ of $\eta$ and make use of the equality $\left.(\mathbb{C} \otimes \eta)_{\mathbb{R}}=\eta \oplus \eta\right)$. On the other hand, if $1 \leq n \leq 3$, then each topological $\mathbb{R}$-vector bundle on $\mathbb{T}^{n}$ is isomorphic to an algebraic $\mathbb{R}$-vector bundle [13].

Assume now that $\mathbb{F}=\mathbb{C}$ or $\mathbb{F}=\mathbb{H}$. We choose a smooth $\mathbb{F}$-line bundle $\theta^{\mathbb{F}}$ on $\mathbb{T}^{d(\mathbb{F})}$ such that $c_{1}\left(\theta^{\mathbb{C}}\right) \neq 0$ and $c_{2}\left(\left(\theta^{\mathbb{H}}\right) \mathbb{C}\right) \neq 0$. If $n \geq d(\mathbb{F})$ and $p_{\mathbb{F}}: \mathbb{T}^{n}=\mathbb{T}^{d(\mathbb{F})} \times \mathbb{T}^{n-d(\mathbb{F})} \rightarrow \mathbb{T}^{d(\mathbb{F})}$ is the canonical projection, then the smooth $\mathbb{F}$-line bundle

$$
\lambda^{\mathbb{F}}:=p_{\mathbb{F}}^{*} \theta^{\mathbb{F}}
$$

on $\mathbb{T}^{n}$ is not topologically isomorphic to any algebraic $\mathbb{F}$-line bundle. This assertion holds since $c_{1}\left(\lambda^{\mathbb{C}}\right) \neq 0$ and $c_{2}\left(\left(\lambda^{\mathbb{H}}\right)_{\mathbb{C}}\right) \neq 0$. Obviously, $\theta^{\mathbb{F}}$ is adapted, and hence $\lambda^{\mathbb{F}}$ is adapted too. Furthermore, the $\mathbb{R}$-vector bundle $\left(\lambda^{\mathbb{C}}\right)_{\mathbb{R}}$ on $\mathbb{T}^{n}$ is isomorphic to an algebraic $\mathbb{R}$-vector bundle since the $\mathbb{R}$-vector bundle $\left(\theta^{\mathbb{C}}\right)_{\mathbb{R}}$ on $\mathbb{T}^{2}$ is isomorphic to an algebraic $\mathbb{R}$-vector bundle, $p_{\mathbb{C}}$ is a regular map, and

$$
\left(\lambda^{\mathbb{C}}\right)_{\mathbb{R}}=p_{\mathbb{C}}^{*}\left(\left(\theta^{\mathbb{C}}\right)_{\mathbb{R}}\right) .
$$

Finally, if $\xi=\left(\lambda^{\mathbb{H}}\right)_{\mathbb{R}}$, then the $\mathbb{R}$-vector bundle $\xi \oplus \xi$ on $\mathbb{T}^{n}$ is not isomorphic to any algebraic $\mathbb{R}$-vector bundle. Indeed, supposing otherwise, the complexification $\mathbb{C} \otimes(\xi \oplus \xi)$ of $\xi \oplus \xi$ would be isomorphic to an algebraic $\mathbb{C}$-vector bundle, and hence $c_{2}(\mathbb{C} \otimes(\xi \oplus \xi))=0$. However, one has $c_{1}\left(\left(\lambda^{\mathbb{H}} \oplus \lambda^{\mathbb{H}}\right)_{\mathbb{C}}\right)=0$, which implies

$$
c_{2}(\mathbb{C} \otimes(\xi \oplus \xi))=c_{2}\left(\mathbb{C} \otimes\left(\lambda^{\mathbb{H}} \oplus \lambda^{\mathbb{H}}\right)_{\mathbb{R}}\right)=2 c_{2}\left(\left(\lambda^{\mathbb{H}} \oplus \lambda^{\mathbb{H}}\right)_{\mathbb{C}}\right)=4 c_{2}\left(\left(\lambda^{\mathbb{H}}\right)_{\mathbb{C}}\right) \neq 0,
$$

cf. [39, Corollary 15.5]. 
There are other significant differences between algebraic and stratified-algebraic vector bundles. The interested reader can identify them by consulting papers devoted to algebraic vector bundles on real algebraic varieties [5, 6, 7, 8, 9, 10, 13, 14]. It should be mentioned that there are topological $\mathbb{F}$-line bundles which are not isomorphic to stratified-algebraic $\mathbb{F}$-line bundles. For instance, such $\mathbb{F}$-line bundles exist on some nonsingular real algebraic varieties diffeomorphic to $\mathbb{T}^{n}$, cf. Example 7.10 .

The paper is organized as follows. In Section 2, we define in a natural way stratifiedregular maps. Some homotopical properties of such maps imply Theorem 1.3. In Section3, we study relationships between vector bundles of various types and appropriate finitely generated projective modules. As a model serve classical results due to Serre [42] and Swan [44]. Subsequently, we prove Theorems 1.5 and 1.6 by applying [45] and some results from K-theory. Section 4 is devoted to maps with values in a Grassmannian (actually, a multi-Grassmannian). The main topic is the approximation of continuous maps by stratified-regular maps. Approximation results of this kind are essential for the rest of the paper. As the starting point for these results the extension theorem of Kollár and Nowak [32] is indispensable. In Section 5, we show how certain properties of a vector bundle can be deduced from the behavior of its restrictions to Zariski closed subvarieties of the base space. This is useful in proofs by induction in Section 6. In particular, Theorems 1.7, 1.8 and 1.9 are proved in Section 6. Algebraic and $\mathbb{C}$-algebraic cohomology classes have many applications in real algebraic geometry, cf. [1, 2, 5, 6, 8, 9, 10, 12, 13, 14, 16, 17, 18, 19, 33, 35. We introduce stratified-algebraic and stratified$\mathbb{C}$-algebraic cohomology classes in Section 7 and Section 8, respectively. They prove to be very useful in our investigation of stratified-algebraic vector bundles. In particular, stratified-C-algebraic cohomology classes play a key role in the proof of Theorem 1.10 given in Section 8 .

Acknowledgements. We greatly benefited from studying [23] and [32], as well as earlier versions of these papers. Our discussions with G. Fichou, J. Huisman, F. Mangolte and J.-Ph. Monnier about their paper [23] provided additional inspiration.

\section{Stratified-regular maps}

Throughout this section, $X$ and $Y$ denote real algebraic varieties, and $\mathcal{X}$ denotes a stratification of $X$.

To begin with, we introduce maps that will be crucial for the investigation of $\mathcal{X}$ algebraic and stratified-algebraic vector bundles.

Definition 2.1. A map $f: X \rightarrow Y$ is said to be $\mathcal{X}$-regular if it is continuous and its restriction to each stratum in $\mathcal{X}$ is a regular map. Furthermore, $f$ is said to be stratifiedregular if it is $\mathcal{S}$-regular for some stratification $\mathcal{S}$ of $X$.

In particular, $f$ is $\{X\}$-regular if and only if it is a regular map. Following [32, 33, 35], we say that $f: X \rightarrow Y$ is a continuous rational map if $f$ is continuous and its restriction to some Zariski open and dense subvariety of $X$ is a regular map.

By a filtration of $X$ we mean a finite sequence $\mathcal{F}=\left(X_{-1}, X_{0}, \ldots, X_{m}\right)$ of Zariski closed subvarieties satisfying

$$
\varnothing=X_{-1} \subseteq X_{0} \subseteq \ldots \subseteq X_{m}=X .
$$

The collection $\overline{\mathcal{F}}:=\left\{X_{i} \backslash X_{i-1} \mid 0 \leq i \leq m\right\}$ is a stratification of $X$. 
Proposition 2.2. For a map $f: X \rightarrow Y$, the following conditions are equivalent:

(a) The map $f$ is stratified-regular.

(b) There exists a filtration $\mathcal{F}$ of $X$ such that $f$ is $\overline{\mathcal{F}}$-regular and each stratum in $\overline{\mathcal{F}}$ is nonsingular and equidimensional.

$\left(\mathrm{b}^{\prime}\right)$ There exists a filtration $\mathcal{F}^{\prime}$ of $X$ such that $f$ is $\overline{\mathcal{F}}^{\prime}$-regular.

(c) For each Zariski closed subvariety $Z$ of $X$, the restriction $\left.f\right|_{Z}: Z \rightarrow Y$ is a continuous rational map.

Proof. Suppose that condition (c) holds. Since the map $f$ is continuous rational, there exists a Zariski open and dense subvariety $X^{0}$ of $X$ such that the restriction of $f$ to $X^{0}$ is a regular map. We can choose such an $X^{0}$ disjoint from the singular locus of $X$. Let $Z$ be the union of $X \backslash X^{0}$ and all the irreducible components of $X$ of dimension strictly less than $\operatorname{dim} X$. Then $Z$ is a Zariski closed subvariety of $X$ with $\operatorname{dim} Z<\operatorname{dim} X$, and $X \backslash Z$ is nonsingular of pure dimension. Since the map $\left.f\right|_{Z}$ is continuous rational, the construction above can be repeated with $X$ replaced by $Z$. By continuing this process, we conclude that $(\mathrm{b})$ holds.

If $V$ is an irreducible Zariski closed subvariety of $X$, then there exists a stratum $S$ in $\mathcal{X}$ such that the intersection $S \cap V$ is nonempty and Zariski open in $V$ (hence Zariski dense in $V$ ). It follows that (a) implies (c).

It is obvious that (b) implies $\left(\mathrm{b}^{\prime}\right)$, and $\left(\mathrm{b}^{\prime}\right)$ implies (a).

Remark 2.3. Proposition 2.2 (with $Y=\mathbb{R}$ ) appears in the paper of Kollár and Nowak 32 as a comment following Definition 8. In [32, the attention is focused on functions satisfying condition (c), called continuous hereditarily rational functions. By [32, Proposition 7], if the variety $X$ is nonsingular, then condition (c) is equivalent to

$\left(\mathrm{c}^{\prime}\right)$ The map $f$ is continuous rational.

If $X$ is singular, then (c') need not imply (c), cf. [32, Examples 2 and 3]. Continuous rational maps defined on nonsingular real algebraic varieties are investigated in [33, 35]. According to [32, Theorem 9] and [23, Corolaire 4.40], stratified-regular maps coincide with "applications régulues" between real algebraic varieties, which are introduced in a more general framework in [23]. Notions introduced and methods developed in [36, 37, 38, 40] are important in the study of the geometry of "fonctions régulues", cf. [23].

The proof of Theorem 1.3 requires some knowledge of homotopy classes represented by stratified-regular maps with values in the unit $d$-sphere $\mathbb{S}^{d}$, cf. Theorem 2.5 . We first need the following technical result.

Lemma 2.4. Assume that the variety $X$ is compact of dimension $d$. Let $A$ be the union of the singular locus of $X$ and all the irreducible components of $X$ of dimension at most $d-1$. Let $\varphi: X \rightarrow \mathbb{R}^{d}$ be a continuous map which is constant in a neighborhood of $A$ and smooth on $X \backslash A$. Assume that 0 in $\mathbb{R}^{d}$ is a regular value of $\left.\varphi\right|_{X \backslash A}$, and the inverse image $V:=\varphi^{-1}(0)$ is disjoint from $A$. Then there exists a regular map $\psi: X \rightarrow \mathbb{R}^{d}$ such that $\psi$ is equal to $\varphi$ on $A, \psi^{-1}(0)=V$, and the differentials $d \psi_{x}$ and $d \varphi_{x}$ are equal for every point $x$ in $V$. 
Proof. We may assume that $X$ is a Zariski closed subvariety of $\mathbb{R}^{n}$ for some $n$. Then we can find a smooth map $f: \mathbb{R}^{n} \rightarrow \mathbb{R}^{d}$ which is an extension of $\varphi$ and is constant on a neighborhood $U$ of $A$ in $\mathbb{R}^{n}$. Thus $U \subseteq f^{-1}(c)$ for some point $c=\left(c_{1}, \ldots, c_{d}\right)$ in $\mathbb{R}^{d}$. Since $V$ is a finite set, there exists a polynomial map $g: \mathbb{R}^{n} \rightarrow \mathbb{R}^{d}$ such that $g(x)=f(x)$ and $d g_{x}=d f_{x}$ for every point $x$ in $V$, and $A \subseteq g^{-1}(c)$. Indeed, $g$ can be constructed as follows. First we choose a polynomial map $\eta: \mathbb{R}^{n} \rightarrow \mathbb{R}^{d}$ with $\eta(x)=f(x)$ and $d \eta_{x}=d f_{x}$ for every $x$ in $V$. If $\alpha: \mathbb{R}^{n} \rightarrow \mathbb{R}$ is a polynomial function satisfying $V \subseteq \alpha^{-1}(0)$ and $A \subseteq \alpha^{-1}(1)$, then the map $g:=\eta-\alpha^{2}(\eta-c)$ satisfies all the requirements.

For any subset $Z$ of $\mathbb{R}^{n}$, we denote by $I(Z)$ the ideal of all polynomial functions on $\mathbb{R}^{n}$ vanishing on $Z$. Let $p_{1}, \ldots, p_{r}$ be generators of the ideal $I(V)^{2} I(A)$.

Assertion. There exist smooth maps $\lambda_{1}: \mathbb{R}^{n} \rightarrow \mathbb{R}^{d}, \ldots, \lambda_{r}: \mathbb{R}^{n} \rightarrow \mathbb{R}^{d}$ such that $f=$ $g+p_{1} \lambda_{1}+\cdots+p_{r} \lambda_{r}$.

The Assertion can be proved as follows. For any point $x$ in $\mathbb{R}^{n}$, denote by $\mathcal{C}_{x}^{\infty}$ the ring of germs at $x$ of smooth functions on $\mathbb{R}^{n}$. Let $f=\left(f_{1}, \ldots, f_{d}\right), g=\left(g_{1}, \ldots, g_{d}\right)$ and $h_{i}=f_{i}-g_{i}$ for $1 \leq i \leq d$. For every point $x$ in $\mathbb{R}^{n} \backslash A$, the germ $\left(h_{i}\right)_{x}$ of $h_{i}$ at $x$ belongs to the ideal $I(V)^{2} I(A) \mathcal{C}_{x}^{\infty}$. Obviously, $g_{i}-c_{i}$ belongs to $I(A)$. If $x$ is a point in $A$, then $f_{i}-c_{i}$ vanishes in a neighborhood of $x$, and hence $\left(h_{i}\right)_{x}=\left(\left(f_{i}\right)_{x}-c_{i}\right)-\left(\left(g_{i}\right)_{x}-c_{i}\right)$ belongs to $I(V)^{2} I(A) \mathcal{C}_{x}^{\infty}$. Making use of partition of unity, we obtain smooth functions $\lambda_{i 1}, \ldots, \lambda_{i r}$ on $\mathbb{R}^{n}$ for which $h_{i}=p_{1} \lambda_{i 1}+\cdots+p_{r} \lambda_{i r}$. Thus, the Assertion holds with $\lambda_{j}=\left(\lambda_{1 j}, \ldots \lambda_{d j}\right)$ for $1 \leq j \leq r$.

Now, let $q_{1}: \mathbb{R}^{n} \rightarrow \mathbb{R}^{d}, \ldots, q_{r}: \mathbb{R}^{n} \rightarrow \mathbb{R}^{d}$ be polynomial maps and let $\psi: X \rightarrow \mathbb{R}^{d}$ be the restriction of the map $g+p_{1} q_{1}+\cdots p_{r} q_{r}$. Then $\psi=\varphi$ on $A, V \subseteq \psi^{-1}(0)$, and $d \psi_{x}=d \varphi_{x}$ for every point $x$ in $V$. According to the Stone-Weierstrass theorem, given $\varepsilon>0$, we can find polynomial maps $q_{j}$ such that

$$
\left\|\lambda_{j}(x)-q_{j}(x)\right\|+\sum_{k=1}^{n}\left\|\frac{\partial \lambda_{j}}{\partial x_{k}}(x)-\frac{\partial q_{j}}{\partial x_{k}}(x)\right\|<\varepsilon
$$

for all $x$ in $X$ and $1 \leq j \leq r$. If $\varepsilon$ is sufficiently small, then $\psi^{-1}(0)=V$, and hence $\psi$ satisfies all the requirements.

Theorem 2.5. If the variety $X$ is compact of dimension $d$, then each continuous map from $X$ into $\mathbb{S}^{d}$ is homotopic to a stratified-regular map.

Proof. Let $A$ be the union of the singular locus of $X$ and all the irreducible components of $X$ of dimension at most $d-1$. Since $(X, A)$ is a polyhedral pair [9, Corollary 9.3.7], the restriction of $f$ to some neighborhood of $A$ is null homotopic. Hence, according to the homotopy extension theorem [28, p. 90, Theorem 1.4], the map $f$ can be deformed without affecting its homotopy class so that $f$ is constant in a compact neighborhood $L$ of $A$. Furthermore, we may assume that $f$ is smooth on $X \backslash A$. By Sard's theorem, there exists a regular value $y$ in $\mathbb{S}^{d}$ of the smooth map $\left.f\right|_{X \backslash A}$ such that both points $y$ and $-y$ are in $\mathbb{S}^{d} \backslash f(L)$. In particular, the set $f^{-1}(y)$ is finite. We choose a compact neighborhood $K$ of $f^{-1}(y)$ in $X$ which is disjoint from $L \cup f^{-1}(-y)$ and such that each point in $K$ is a regular point of $f$. Let $p: \mathbb{S}^{d} \backslash\{-y\} \rightarrow \mathbb{R}^{d}$ be the stereographic projection (in particular, $p(y)=0)$. Let $\kappa: X \rightarrow \mathbb{R}$ be a continuous function, smooth on $X \backslash A$, with $\kappa=1$ on $K \cup L$ and $\kappa=0$ in a neighborhood of $f^{-1}(-y)$. The map $\xi=\left(\xi_{1}, \ldots, \xi_{d}\right): X \rightarrow \mathbb{R}^{d}$, defined by

$$
\xi(x)= \begin{cases}\kappa(x) p(f(x)) & \text { for } x \text { in } X \backslash f^{-1}(-y) \\ 0 & \text { for } x \text { in } f^{-1}(-y),\end{cases}
$$


is continuous, smooth on $X \backslash A, \xi^{-1}(0) \cap L=\varnothing, f^{-1}(y) \subseteq \xi^{-1}(0)$, and each point in $K$ is a regular point of $\xi$. Let $\lambda: X \rightarrow \mathbb{R}$ be a continuous function, smooth on $X \backslash A$, with $\lambda^{-1}(0)=K \cup L$ (such a function exists, cf. for instance [21, Theorem 14.1]). The map $\eta: X \times \mathbb{R}^{d} \rightarrow \mathbb{R}^{d}$, defined by

$$
\eta\left(x,\left(s_{1}, \ldots s_{d}\right)\right)=\left(\xi_{1}(x)+s_{1} \lambda(x)^{2}, \ldots, \xi_{d}(x)+s_{d} \lambda(x)^{2}\right),
$$

is continuous, smooth on $(X \backslash A) \times \mathbb{R}^{d}$, and 0 in $\mathbb{R}^{d}$ is a regular value of the restriction of $\eta$ to $(X \backslash A) \times \mathbb{R}^{d}$. According to the parametric transversality theorem, we can choose a point $\left(s_{1}, \ldots, s_{d}\right)$ in $\mathbb{R}^{d}$ such that if $\varphi: X \rightarrow \mathbb{R}^{d}$ is defined by $\varphi(x)=\eta\left(x,\left(s_{1}, \ldots, s_{d}\right)\right)$ for all $x$ in $X$, then 0 in $\mathbb{R}^{d}$ is a regular value of the restriction of $\varphi$ to $X \backslash A$. By construction, $\varphi$ is constant in a neighborhood of $A$, and the set $V:=\varphi^{-1}(0)$ is disjoint from $A$. Furthermore, $V$ is a finite set containing $f^{-1}(y)$. Let $\psi: X \rightarrow \mathbb{R}^{d}$ be a regular map as in Lemma 2.4. We choose a regular function $\alpha: X \rightarrow \mathbb{R}$ with $\alpha^{-1}(0)=W$ and $\alpha^{-1}(1)=f^{-1}(y) \cup A$, where $W:=V \backslash f^{-1}(y)$. For example, we can take $\alpha=\alpha_{1}^{2} /\left(\alpha_{1}^{2}+\alpha_{2}^{2}\right)$, where $\alpha_{1}$ and $\alpha_{2}$ are regular functions on $X$ satisfying $\alpha_{1}^{-1}(0)=W$ and $\alpha_{2}^{-1}(0)=f^{-1}(y) \cup A$. By the Łojasiewicz inequality [9, Corollary 2.6.7], there exist a neighborhood $U$ of $W$ in $X$, a positive real number $c$, and a positive integer $k$ for which

$$
\|\psi(x)\| \geq c \alpha(x)^{2 k} \text { for all } x \text { in } U .
$$

Let $\bar{\psi}(x)=\left(1 / \alpha(x)^{2 k+1}\right) \psi(x)$ for $x$ in $X \backslash W$. The map $g: X \rightarrow \mathbb{S}^{d}$, defined by

$$
g(x)= \begin{cases}p^{-1}(\bar{\psi}(x)) & \text { for } x \text { in } X \backslash W \\ -y & \text { for } x \text { in } W\end{cases}
$$

is continuous. Since $p$ is a biregular isomorphism, the restriction $\left.g\right|_{X \backslash W}$ is a regular map, and hence $g$ is a stratified-regular map. It suffices to prove that $f$ is homotopic to $g$.

The map $\bar{\psi}: X \backslash W \rightarrow \mathbb{R}^{d}$ has the following properties: $\psi^{-1}(0)=f^{-1}(y)=V \backslash W$ and $d \bar{\psi}_{x}=d \varphi_{x}$ for every point $x$ in $f^{-1}(y)$. If $U_{1}$ is a sufficiently small open neighborhood of $f^{-1}(y)$ in $X$, then

$$
(1-t) \varphi(x)+t \bar{\psi}(x) \neq 0 \quad \text { for all }(x, t) \text { in }\left(U_{1} \backslash f^{-1}(y)\right) \times[0,1] .
$$

We may assume that $U_{1} \subseteq K$, and hence $\varphi(x)=p(f(x))$ for all $x$ in $U_{1}$. Let $U_{2}$ be an open neighborhood of $f^{-1}(y)$ whose closure $\overline{U_{2}}$ is contained in $U_{1}$. Choose a continuous function $\mu: X \rightarrow[0,1]$, smooth on $X \backslash A$, with $\mu=1$ on $\overline{U_{2}}$ and $\mu=0$ in a neighborhood of $X \backslash U_{1}$. Then the map $F: X \times[0,1] \rightarrow \mathbb{S}^{p}$, defined by

$$
F(x, t)= \begin{cases}p^{-1}((1-t \mu(x)) \varphi(x)+t \mu(x) \bar{\psi}(x)) & \text { for }(x, t) \text { in } U_{1} \times[0,1] \\ f(x) & \text { for }(x, t) \text { in }\left(X \backslash U_{1}\right) \times[0,1]\end{cases}
$$

is continuous. Furthermore, if $F_{t}: X \rightarrow \mathbb{S}^{p}$ is defined by $F_{t}(x)=F(x, t)$, then $F_{t}^{-1}(y)=$ $f^{-1}(y)=g^{-1}(y)$ for every $t$ in $[0,1]$, and $F_{0}=f$. It remains to prove that the map $\bar{f}:=F_{1}$ is homotopic to $g$. This can be done as follows. Note that $\bar{f}=g$ on $U_{2}$. If $q: \mathbb{S}^{d} \backslash\{y\} \rightarrow \mathbb{R}^{d}$ is the stereographic projection, then

$$
G(x, t)= \begin{cases}q^{-1}((1-t) q(\bar{f}(x))+t q(g(x))) & \text { for }(x, t) \text { in }\left(X \backslash f^{-1}(y)\right) \times[0,1] \\ \bar{f}(x) & \text { for }(x, t) \text { in } U_{2} \times[0,1]\end{cases}
$$

is a homotopy between $\bar{f}$ and $g$. 
A special case of Theorem 2.5, with $X$ nonsingular, is contained in [33, Theorem 1.2].

Recall that each regular map from $\mathbb{T}^{n}$ into $\mathbb{S}^{n}$ is null homotopic, cf. [11] or [12]. In particular, Theorem 2.5 shows that stratified-regular maps are more flexible than regular maps. Other results illustrating this point can be found in [33, 35.

For any continuous map $f: X \rightarrow Y$ and any topological $\mathbb{F}$-vector subbundle $\theta$ of $\varepsilon_{Y}^{n}(\mathbb{F})$, the pullback $f^{*} \theta$ will be regarded as a topological $\mathbb{F}$-vector subbundle of $\varepsilon_{X}^{n}(\mathbb{F})$.

Proposition 2.6. If the map $f$ is $\mathcal{X}$-regular, and the $\mathbb{F}$-vector bundle $\theta$ is algebraic, then $f^{*} \theta$ is $\mathcal{X}$-algebraic. Similarly, if $f$ is stratified-regular and $\theta$ is stratified-algebraic, then $f^{*} \theta$ is stratified-algebraic.

Proof. The first assertion is obvious. For the second assertion, let $\mathcal{T}$ be a stratification of $X$ such that the map $f$ is $\mathcal{T}$-regular, and let $\mathcal{Y}$ be a stratification of $Y$ such that the $\mathbb{F}$ vector bundle $\theta$ is $\mathcal{Y}$-algebraic. Then $\mathcal{S}:=\left\{T \cap f^{-1}(P) \mid T \in \mathcal{T}, P \in \mathcal{Y}\right\}$ is a stratification of $X$, and $f^{*} \theta$ is $\mathcal{S}$-algebraic.

Proof of Theorem 1.3. Let $h: X \rightarrow \mathbb{S}^{d}$ be a homotopy equivalence. By Theorem 2.5, $h$ is homotopic to a stratified-regular map $f: X \rightarrow \mathbb{S}^{d}$. According to Proposition 2.6, if $\theta$ is an algebraic $\mathbb{F}$-vector bundle on $\mathbb{S}^{d}$, then $f^{*} \theta$ is a stratified-algebraic $\mathbb{F}$-vector bundle on $X$. The proof is complete since every topological $\mathbb{F}$-vector bundle on $\mathbb{S}^{d}$ is isomorphic to an algebraic $\mathbb{F}$-vector bundle (cf. Example 1.4).

\section{Basic properties of stratified-algebraic vector bundles}

Throughout this section, $X$ denotes a real algebraic variety, and $\mathcal{X}$ is a stratification of $X$. All modules that appear below are left modules.

Vector bundles are often investigated by means of maps into Grassmannians, cf. [3, 5, 9, 10, 26, 28, 29, 30]. As in [9, 10], the Grassmannian $\mathbb{G}_{k}\left(\mathbb{F}^{n}\right)$ of $k$-dimensional $\mathbb{F}$-vector subspaces of $\mathbb{F}^{n}$ will be regarded as a real algebraic variety. The tautological $\mathbb{F}$-vector bundle on $\mathbb{G}_{k}\left(\mathbb{F}^{n}\right)$ will be denoted by $\gamma_{k}\left(\mathbb{F}^{n}\right)$. If $\xi$ is a topological (resp. algebraic) $\mathbb{F}$-vector subbundle of $\varepsilon_{X}^{n}(\mathbb{F})$ of rank $k$, then the map $f: X \rightarrow \mathbb{G}_{k}\left(\mathbb{F}^{n}\right)$ defined by

$$
E(\xi)_{x}=\{x\} \times f(x) \text { for all } x \text { in } X
$$

is continuous (resp. regular).

Let $K$ be a finite nonempty collection of nonnegative integers and $n$ an integer such that $n \geq k$ for every $k$ in $K$. We denote by $\mathbb{G}_{K}\left(\mathbb{F}^{n}\right)$ the disjoint union of all $\mathbb{G}_{k}\left(\mathbb{F}^{n}\right)$ for $k$ in $K$. The tautological $\mathbb{F}$-vector bundle $\gamma_{K}\left(\mathbb{F}^{n}\right)$ on $\mathbb{G}_{K}\left(\mathbb{F}^{n}\right)$ is the bundle whose restriction to $\mathbb{G}_{k}\left(\mathbb{F}^{n}\right)$ is $\gamma_{k}\left(\mathbb{F}^{n}\right)$ for each $k$ in $K$. In particular, $\mathbb{G}_{K}\left(\mathbb{F}^{n}\right)$ is a real algebraic variety, and $\gamma_{K}\left(\mathbb{F}^{n}\right)$ is an algebraic $\mathbb{F}$-vector subbundle of the standard trivial $\mathbb{F}$-vector bundle on $\mathbb{G}_{K}\left(\mathbb{F}^{n}\right)$ with fiber $\mathbb{F}^{n}$. We call $\mathbb{G}_{K}\left(\mathbb{F}^{n}\right)$ the $(K, n)$-multi-Grassmannian. It is explained below why we need this notion.

Any algebraic $\mathbb{F}$-vector bundle on $X$ has constant rank on each Zariski connected component of $X$. This observation can be partially generalized as follows.

Proposition 3.1. Assume that the variety $X$ is nonsingular. Then any stratified-algebraic $\mathbb{F}$-vector bundle on $X$ has constant rank on each irreducible component of $X$. 
Proof. We may assume that $X$ is irreducible. It suffices to note that in each stratification of $X$, one can find a stratum which is nonempty and Zariski open in $X$.

However, we encounter a different phenomenon for vector bundles on singular varieties.

Example 3.2. The real algebraic curve

$$
C:=\left\{(x, y) \in \mathbb{R}^{2} \mid y^{2}=x^{2}(x-1)\right\}
$$

is irreducible, and hence Zariski connected. It has two connected components in the Euclidean topology, $S_{1}=\{(0,0)\}$ and $S_{2}=C \backslash S_{1}$. The collection $\mathcal{C}=\left\{S_{1}, S_{2}\right\}$ is a stratification of $C$. Let $\xi$ be the topological $\mathbb{F}$-vector subbundle of $\varepsilon_{C}^{2}(\mathbb{F})$ such that $E\left(\left.\xi\right|_{S_{1}}\right)=\{(0,0)\} \times(\mathbb{F} \times\{0\})$ and $\left.\xi\right|_{S_{2}}=\varepsilon_{S_{2}}^{2}(\mathbb{F})$. Then $\xi$ is $\mathcal{C}$-algebraic and it does not have constant rank. Furthermore, if $K=\{1,2\}$ and $f: C \rightarrow \mathbb{G}_{K}\left(\mathbb{F}^{2}\right)$ is a map such that $f\left(S_{1}\right) \subseteq \mathbb{G}_{1}\left(\mathbb{F}^{2}\right)$ and $f\left(S_{2}\right) \subseteq \mathbb{G}_{2}\left(\mathbb{F}^{2}\right)$, then $f$ is $\mathcal{C}$-regular with $\xi=f^{*} \gamma_{K}\left(\mathbb{F}^{2}\right)$.

Proposition 3.3. Any $\mathcal{X}$-algebraic $\mathbb{F}$-vector bundle $\xi$ on $X$ is of the form $\xi=f^{*} \gamma_{K}\left(\mathbb{F}^{n}\right)$ for some multi-Grassmannian $\mathbb{G}_{K}\left(\mathbb{F}^{n}\right)$ and $\mathcal{X}$-regular map $f: X \rightarrow \mathbb{G}_{K}\left(\mathbb{F}^{n}\right)$.

Proof. Let $\xi$ be an $\mathcal{X}$-algebraic $\mathbb{F}$-vector subbundle of $\varepsilon_{X}^{n}(\mathbb{F})$. For each stratum $S$ in $\mathcal{X}$, the function

$$
S \rightarrow \mathbb{Z}, \quad x \rightarrow \operatorname{dim}_{\mathbb{F}} E(\xi)_{x}
$$

is locally constant in the Zariski topology, the $\mathbb{F}$-vector bundle $\left.\xi\right|_{S}$ being algebraic. Hence, the set $K:=\left\{\operatorname{dim}_{\mathbb{F}} E(\xi)_{x} \mid x \in X\right\}$ is finite. The map $f: X \rightarrow \mathbb{G}_{K}\left(\mathbb{F}^{n}\right)$ defined by

$$
E(\xi)_{x}=\{x\} \times f(x) \text { for all } x \text { in } X
$$

is continuous and $\xi=f^{*} \gamma_{K}\left(\mathbb{F}^{n}\right)$. Furthermore, the map $\left.f\right|_{S}$ is regular. Thus $f$ is $\mathcal{X}$ regular, as required.

Proposition 3.4. Any stratified-algebraic $\mathbb{F}$-vector bundle $\xi$ on $X$ is of the form

$$
\xi=f^{*} \gamma_{K}\left(\mathbb{F}^{n}\right)
$$

for some multi-Grassmannian $\mathbb{G}_{K}\left(\mathbb{F}^{n}\right)$ and stratified-regular map $f: X \rightarrow \mathbb{G}_{K}\left(\mathbb{F}^{n}\right)$.

Proof. It suffices to apply Proposition 3.3.

Proposition 3.5. For a topological $\mathbb{F}$-vector bundle $\xi$ on $X$, the following conditions are equivalent:

(a) The bundle $\xi$ is stratified-algebraic.

(b) There exists a filtration $\mathcal{F}$ of $X$ such that $\xi$ is $\overline{\mathcal{F}}$-algebraic and each stratum in $\overline{\mathcal{F}}$ is nonsingular and equidimensional.

$\left(\mathrm{b}^{\prime}\right)$ There exists a filtration $\mathcal{F}^{\prime}$ of $X$ such that $\xi$ is $\overline{\mathcal{F}}^{\prime}$-algebraic.

Proof. By Proposition 3.4, if the $\mathbb{F}$-vector bundle $\xi$ is stratified-algebraic, then

$$
\xi=f^{*} \gamma_{K}\left(\mathbb{F}^{n}\right)
$$

for some multi-Grassmannian $\mathbb{G}_{K}\left(\mathbb{F}^{n}\right)$ and stratified-regular map $f: X \rightarrow \mathbb{G}_{K}\left(\mathbb{F}^{n}\right)$. According to Proposition 2.2 , there exists a filtration $\mathcal{F}$ of $X$ such that $f$ is $\overline{\mathcal{F}}$-regular and each stratum in $\overline{\mathcal{F}}$ is nonsingular and equidimensional. It follows that $\xi$ is $\overline{\mathcal{F}}$-algebraic. Consequently, (a) implies (b). It is obvious that $(\sqrt{b})$ implies $\left(\sqrt{b^{\prime}}\right)$, and $\left(\sqrt{b^{\prime}}\right)$ implies $(a)$. 
Recall that $d(\mathbb{F})=\operatorname{dim}_{\mathbb{R}} \mathbb{F}$. As a consequence of Theorem 2.5, we obtain the following result on vector bundles on low-dimensional varieties.

Corollary 3.6. Assume that the variety $X$ is compact and $\operatorname{dim} X \leq d(\mathbb{F})$. Then any topological $\mathbb{F}$-vector bundle of constant rank on $X$ is topologically isomorphic to a stratifiedalgebraic $\mathbb{F}$-vector bundle.

Proof. Let $\xi$ be a topological $\mathbb{F}$-vector bundle of rank $r \geq 1$ on $X$. Since $\operatorname{dim} X \leq d(\mathbb{F})$, the bundle $\xi$ splits off a trivial vector bundle of rank $r-1$. Moreover, if $\operatorname{dim} X<d(\mathbb{F})$, then $\xi$ is topologically trivial. These are well known topological facts, cf. [29, p. 99]. Hence we may assume without loss of generality that $r=1$ and $\operatorname{dim} X=d(\mathbb{F})$. Then there exists a continuous map $f: X \rightarrow \mathbb{G}_{1}\left(\mathbb{F}^{2}\right)$ such that the pullback $\mathbb{F}$-line bundle $f^{*} \gamma_{1}\left(\mathbb{F}^{2}\right)$ is isomorphic to $\xi$. Recall that $\mathbb{G}_{1}\left(\mathbb{F}^{2}\right)$ is biregularly isomorphic to the unit $d(\mathbb{F})$-sphere. Consequently, according to Theorem 2.5, the map $f$ can be assumed to be stratified-regular. Thus, it suffices to apply Proposition 2.6.

Subsequent results require some preparation. For any real algebraic variety $Y$, denote by $\mathcal{C}(X, Y)$ the set of all continuous maps from $X$ into $Y$. There are the following inclusions:

$$
\mathcal{R}(X, Y) \subseteq \mathcal{R}_{\mathcal{X}}(X, Y) \subseteq \mathcal{R}^{0}(X, Y) \subseteq \mathcal{C}(X, Y),
$$

where $\mathcal{R}(X, Y)$ (resp. $\mathcal{R}_{\mathcal{X}}(X, Y), \mathcal{R}^{0}(X, Y)$ ) is the set of all regular (resp. $\mathcal{X}$-regular, stratified-regular) maps. Each of the sets $\mathcal{R}(X, \mathbb{F}), \mathcal{R}_{\mathcal{X}}(X, \mathbb{F})$ and $\mathcal{R}^{0}(X, \mathbb{F})$ is a subring of the ring $\mathcal{C}(X, \mathbb{F})$. We next discuss various aspects of the Serre-Swan construction [42, 44, relating vector bundles and finitely generated projective modules.

If $\xi$ is a topological $\mathbb{F}$-vector bundle on $X$, then the set $\Gamma(\xi)$ of all (global) continuous sections of $\xi$ is a $\mathcal{C}(X, \mathbb{F})$-module. If $\varphi: \xi \rightarrow \eta$ is a morphism of topological $\mathbb{F}$-vector bundles on $X$, then

$$
\Gamma(\varphi): \Gamma(\xi) \rightarrow \Gamma(\eta), \quad \Gamma(\varphi)(\sigma)=\varphi \circ \sigma
$$

is a homomorphism of $\mathcal{C}(X, \mathbb{F})$-modules. Since $X$ is homotopically equivalent to a compact subset of $X$ (cf. 9, Corollary 9.3.7]), it follows form [44 that $\Gamma$ is an equivalence of the category of topological $\mathbb{F}$-vector bundles on $X$ with the category of finitely generated projective $\mathcal{C}(X, \mathbb{F})$-modules. We give below suitable counterparts of this result for $\mathcal{X}$ algebraic and stratified-algebraic $\mathbb{F}$-vector bundles on $X$.

If $\xi$ is an $\mathcal{X}$-algebraic $\mathbb{F}$-vector bundle on $X$, an $\mathcal{X}$-algebraic section $u: X \rightarrow \xi$ is a continuous section whose restriction $\left.u\right|_{S}:\left.S \rightarrow \xi\right|_{S}$ to each stratum $S$ in $\mathcal{X}$ is an algebraic section. In other words, $u: X \rightarrow E(\xi)$ is a continuous map such that $\pi(\xi) \circ u$ is the identity map of $X$, and the restriction $\left.u\right|_{S}: S \rightarrow \pi(\xi)^{-1}(S)$ is a regular map of real algebraic varieties for each stratum $S$ in $\mathcal{X}$. The set $\Gamma_{\mathcal{X}}(\xi)$ of all (global) $\mathcal{X}$-algebraic sections of $\xi$ is an $\mathcal{R}_{\mathcal{X}}(X, \mathbb{F})$-module. For any $\mathcal{X}$-algebraic morphism $\varphi: \xi \rightarrow \eta$ of $\mathcal{X}$-algebraic $\mathbb{F}$-vector bundles on $\mathrm{X}$,

$$
\Gamma_{\mathcal{X}}(\varphi): \Gamma_{\mathcal{X}}(\xi) \rightarrow \Gamma_{\mathcal{X}}(\eta), \quad \Gamma_{\mathcal{X}}(\varphi)(u)=\varphi \circ u
$$

is a homomorphism of $\mathcal{R}_{\mathcal{X}}(X, \mathbb{F})$-modules.

The $\mathcal{R}(X, \mathbb{F})$-module $\Gamma_{\mathcal{X}}\left(\varepsilon_{X}^{n}(\mathbb{F})\right)$ is canonically isomorphic to the $\operatorname{direct} \operatorname{sum} \mathcal{R}_{\mathcal{X}}(X, \mathbb{F})^{n}$ of $n$ copies of $\mathcal{R}_{\mathcal{X}}(X, \mathbb{F})$. If $\xi$ is an $\mathcal{X}$-algebraic $\mathbb{F}$-vector subbundle of $\varepsilon_{X}^{n}(\mathbb{F})$, then $\Gamma_{\mathcal{X}}(\xi)$ will be regarded as a submodule of $\Gamma_{\mathcal{X}}\left(\varepsilon_{X}^{n}(\mathbb{F})\right)$.

For any topological $\mathbb{F}$-vector subbundle $\xi$ of $\varepsilon_{X}^{n}(\mathbb{F})$, let $\xi^{\perp}$ denote its orthogonal complement with respect to the standard inner product $\mathbb{F}^{n} \times \mathbb{F}^{n} \rightarrow \mathbb{F}$. Thus, $\xi^{\perp}$ is a topological $\mathbb{F}$-vector subbundle of $\varepsilon_{X}^{n}(\mathbb{F})$ and $\xi \oplus \xi^{\perp}=\varepsilon_{X}^{n}(\mathbb{F})$. The orthogonal projection $\varepsilon_{X}^{n}(\mathbb{F}) \rightarrow \xi$ is a topological morphism of $\mathbb{F}$-vector bundles. 
Proposition 3.7. If $\xi$ is an $\mathcal{X}$-algebraic $\mathbb{F}$-vector subbundle of $\varepsilon_{X}^{n}(\mathbb{F})$, then $\xi^{\perp}$ also is an $\mathcal{X}$-algebraic $\mathbb{F}$-vector subbundle of $\varepsilon_{X}^{n}(\mathbb{F})$, and the orthogonal projection $\varepsilon_{X}^{n}(\mathbb{F}) \rightarrow \xi$ is an $\mathcal{X}$-algebraic morphism. In particular, $\xi$ is generated by $n$ (global) $\mathcal{X}$-algebraic sections.

Proof. Let $S$ be a stratum in $\mathcal{X}$. One readily checks that $\left(\left.\xi\right|_{S}\right)^{\perp}=\left.\left(\xi^{\perp}\right)\right|_{S}$ is an algebraic $\mathbb{F}$-vector subbundle of $\varepsilon_{S}^{n}(\mathbb{F})=\left.\varepsilon_{X}^{n}(\mathbb{F})\right|_{S}$, and the orthogonal projection $\left.\varepsilon_{S}^{n}(\mathbb{F}) \rightarrow \xi\right|_{S}$ is an algebraic morphism. The last assertion in the proposition follows immediately.

Proposition 3.8. If $\xi$ is an $\mathcal{X}$-algebraic $\mathbb{F}$-vector bundle on $X$, then the $\mathcal{R}_{\mathcal{X}}(X, \mathbb{F})$ module $\Gamma_{\mathcal{X}}(\xi)$ is finitely generated and projective. Furthermore, $\Gamma_{\mathcal{X}}$ is an equivalence of the category of $\mathcal{X}$-algebraic $\mathbb{F}$-vector bundles on $X$ with the category of finitely generated projective $\mathcal{R}_{\mathcal{X}}(X, \mathbb{F})$-modules.

Proof. According to Proposition 3.7, if $\xi$ is an $\mathcal{X}$-algebraic $\mathbb{F}$-vector subbundle of $\varepsilon_{X}^{n}(\mathbb{F})$, then

$$
\Gamma_{\mathcal{X}}(\xi) \oplus \Gamma_{\mathcal{X}}\left(\xi^{\perp}\right)=\Gamma_{\mathcal{X}}\left(\varepsilon_{X}^{n}(\mathbb{F})\right) .
$$

Hence, $\Gamma_{\mathcal{X}}(\xi)$ is a finitely generated projective $\mathcal{R}_{\mathcal{X}}(X, \mathbb{F})$-module. Furthermore, $\Gamma_{\mathcal{X}}$ is an equivalence of categories since, in view of Proposition 3.7, the proof given in [3, pp. 30, 31] that $\Gamma$ is an equivalence of categories in the topological framework can easily be adapted to $\Gamma_{\mathcal{X}}$

If $\xi$ is an algebraic $\mathbb{F}$-vector bundle on $X$, then the set $\Gamma_{\text {alg }}(\xi)$ of all (global) algebraic sections of $\xi$ is an $\mathcal{R}(X, \mathbb{F})$-module. For any morphism $\varphi: \xi \rightarrow \eta$ of algebraic $\mathbb{F}$-vector bundles on $X$,

$$
\Gamma_{\text {alg }}(\varphi): \Gamma_{\text {alg }}(\xi) \rightarrow \Gamma_{\text {alg }}(\eta), \quad \Gamma_{\text {alg }}(\varphi)(\sigma)=\varphi \circ \sigma
$$

is a homomorphism of $\mathcal{R}(X, \mathbb{F})$-modules. It is well known that $\Gamma_{\text {alg }}$ is an equivalence of the category of algebraic $\mathbb{F}$-vector bundles on $X$ with the category of finitely generated projective $\mathcal{R}(X, \mathbb{F})$-modules, cf. [9, Proposition 12.1.12]. This result is a special case of Proposition 3.8 since algebraic $\mathbb{F}$-vector bundles on $X$ coincide with $\{X\}$-algebraic $\mathbb{F}$-vector bundles, and $\mathcal{R}(X, \mathbb{F})=\mathcal{R}_{\{X\}}(X, \mathbb{F})$.

There is also a version of Proposition 3.8 for stratified-algebraic vector bundles. If $\xi$ is a stratified-algebraic $\mathbb{F}$-vector bundle on $X$, a stratified-algebraic section $u: X \rightarrow \xi$ is an $\mathcal{S}$-algebraic section for some stratification $\mathcal{S}$ of $X$ such that $\xi$ is an $\mathcal{S}$-algebraic $\mathbb{F}$-vector bundle. The set $\Gamma_{\text {str }}(\xi)$ of all (global) stratified-algebraic sections of $\xi$ is an $\mathcal{R}^{0}(X, \mathbb{F})$ module. For any stratified-algebraic morphism $\varphi: \xi \rightarrow \eta$ of stratified-algebraic $\mathbb{F}$-vector bundles on $X$,

$$
\Gamma_{\text {str }}(\varphi): \Gamma_{\text {str }}(\xi) \rightarrow \Gamma_{\text {str }}(\eta), \quad \Gamma_{\text {str }}(\varphi)(\sigma)=\varphi \circ \sigma
$$

is a homomorphism of $\mathcal{R}^{0}(X, \mathbb{F})$-modules.

Proposition 3.9. If $\xi$ is a stratified-algebraic $\mathbb{F}$-vector bundle on $X$, then the $\mathcal{R}^{0}(X, \mathbb{F})$ module $\Gamma_{\mathrm{str}}(\xi)$ is finitely generated and projective. Furthermore, $\Gamma_{\mathrm{str}}$ is an equivalence of the category of stratified-algebraic $\mathbb{F}$-vector bundles on $X$ with the category of finitely generated projective $\mathcal{R}^{0}(X, \mathbb{F})$-modules.

Proof. One proceeds as in the proof of Proposition 3.8 .

We identify the direct sum $\varepsilon_{X}^{n}(\mathbb{F}) \oplus \varepsilon_{X}^{m}(\mathbb{F})$ with $\varepsilon_{X}^{n+m}(\mathbb{F})$. Consequently, if $\xi \subseteq \varepsilon_{X}^{n}(\mathbb{F})$ and $\eta \subseteq \varepsilon_{X}^{m}(\mathbb{F})$ are $\mathbb{F}$-vector subbundles, then $\xi \oplus \eta \subseteq \varepsilon_{X}^{n+m}(\mathbb{F})$ is an $\mathbb{F}$-vector subbundle.

It is convenient to bring into play the sets of isomorphism classes of vector bundles of types considered above. Denote by $\operatorname{VB}_{\mathbb{F} \text {-alg }}(X), \operatorname{VB}_{\mathbb{F}-\mathcal{X}}(X), \operatorname{VB}_{\mathbb{F} \text {-str }}(X)$ and $\operatorname{VB}_{\mathbb{F}}(X)$ 
the sets of isomorphism classes (in the appropriate category) of algebraic, $\mathcal{X}$-algebraic, stratified-algebraic and topological $\mathbb{F}$-vector bundles on $X$. Each of these sets of isomorphism classes is a commutative monoid with operation induced by direct sum of $\mathbb{F}$-vector bundles. There are obvious canonical homomorphisms of monoids

$$
\operatorname{VB}_{\mathbb{F} \text {-alg }}(X) \rightarrow \operatorname{VB}_{\mathbb{F}-\mathcal{X}}(X) \rightarrow \operatorname{VB}_{\mathbb{F}-\text { str }}(X) \rightarrow \operatorname{VB}_{\mathbb{F}}(X) .
$$

For example, if $\xi$ is an $\mathcal{X}$-algebraic $\mathbb{F}$-vector bundle on $X$, then $\operatorname{VB}_{\mathbb{F}-\mathcal{X}}(X) \rightarrow \operatorname{VB}_{\mathbb{F} \text {-str }}(X)$ sends the isomorphism class of $\xi$ in the category of $\mathcal{X}$-algebraic $\mathbb{F}$-vector bundles on $X$ to the isomorphism class of $\xi$ in the category of stratified-algebraic $\mathbb{F}$-vector bundles on $X$. Any composition of these homomorphisms will also be called a canonical homomorphism. Note that $\operatorname{VB}_{\mathbb{F} \text {-alg }}(x)=\operatorname{VB}_{\mathbb{F}-\{X\}}(X)$.

For any ring $R$ (associative with 1 ), the set $P(R)$ of isomorphism classes of finitely generated projective $R$-modules is a commutative monoid, with operation induced by direct sum of $R$-modules. If $R$ is a subring of a ring $R^{\prime}$, then there is a canonical homomorphism

$$
P(R) \rightarrow P\left(R^{\prime}\right)
$$

induced by the correspondence which assigns to an $R$-module $M$ the $R^{\prime}$-module $R^{\prime} \otimes_{R} M$.

There are canonical homomorphisms of monoids

$$
\begin{array}{ll}
\Gamma_{\text {alg }}: \operatorname{VB}_{\mathbb{F} \text {-alg }}(X) \rightarrow P(\mathcal{R}(X, \mathbb{F})), & \Gamma_{\mathcal{X}}: \operatorname{VB}_{\mathbb{F}-\mathcal{X}}(X) \rightarrow P\left(\mathcal{R}_{\mathcal{X}}(X, \mathbb{F})\right), \\
\Gamma_{\text {str }}: \operatorname{VB}_{\mathbb{F} \text {-str }}(X) \rightarrow P\left(\mathcal{R}^{0}(X, \mathbb{F})\right), & \Gamma: \operatorname{VB}_{\mathbb{F}}(X) \rightarrow P(\mathcal{C}(X, \mathbb{F})),
\end{array}
$$

induced by the global section functor in the appropriate category of $\mathbb{F}$-vector bundles on $X$. For example, $\Gamma_{\mathcal{X}}: \operatorname{VB}_{\mathbb{F}-\mathcal{X}}(X) \rightarrow P\left(\mathcal{R}_{\mathcal{X}}(X, \mathbb{F})\right)$ sends the isomorphism class of an $\mathcal{X}$-algebraic $\mathbb{F}$-vector bundle $\xi$ on $X$ to the isomorphism class of the $\mathcal{R}_{\mathcal{X}}(X, \mathbb{F})$-module $\Gamma \mathcal{X}(\xi)$ (cf. Proposition 3.8).

Theorem 3.10. The diagram

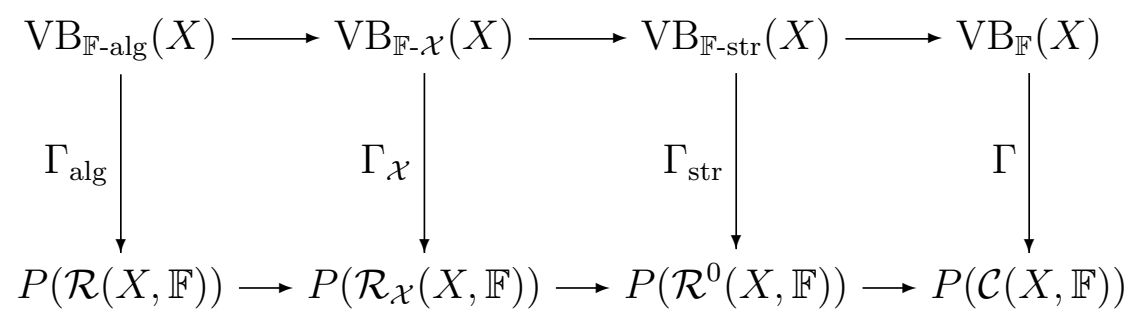

is commutative, and the vertical maps are all bijective. Furthermore, if the variety $X$ is compact, then the horizontal maps are all injective.

Proof. Let $\xi$ be an $\mathcal{X}$-algebraic $\mathbb{F}$-vector subbundle of $\varepsilon_{X}^{n}(\mathbb{F})$. Tensoring

$$
\Gamma_{\mathcal{X}}(\xi) \oplus \Gamma_{\mathcal{X}}\left(\xi^{\perp}\right)=\Gamma_{\mathcal{X}}\left(\varepsilon_{X}^{n}(\mathbb{F})\right)
$$

with $\mathcal{R}^{0}(X, \mathbb{F})$ (over the ring $\mathcal{R}_{\mathcal{X}}(X, \mathbb{F})$ ) and identifying $\mathcal{R}^{0}(X, \mathbb{F}) \otimes \Gamma_{\mathcal{X}}\left(\varepsilon_{X}^{n}(\mathbb{F})\right.$ ) with $\Gamma_{\text {str }}\left(\varepsilon_{X}^{n}(\mathbb{F})\right)$, one readily checks that the $\mathcal{R}^{0}(X, \mathbb{F})$-modules $\mathcal{R}^{0}(X, \mathbb{F}) \otimes \Gamma_{\mathcal{X}}(\xi)$ and $\Gamma_{\text {str }}(\xi)$ are isomorphic. This implies that the middle square in the diagram is commutative. Similar arguments show that the other two squares also are commutative. According to Propositions 3.8 and 3.9, the maps $\Gamma_{\text {alg }}, \Gamma_{\mathcal{X}}$ and $\Gamma_{\text {str }}$ are bijective. By [42], the map $\Gamma$ is bijective (since $X$ is homotopically equivalent to a compact subset of $X$, cf. [9, Corollary 9.3.7]). 
Suppose that the variety $X$ is compact. It suffices to prove that each map in the bottom row of the diagram is injective. This follows from Swan's theorem [45, Theorem 2.2]. Indeed, $\mathcal{C}(X, \mathbb{F})$ is a topological ring with topology induced by the sup norm. Each subring of $\mathcal{C}(X, \mathbb{F})$ is a topological ring with the subspace topology. By the Weierstrass approximation theorem, $\mathcal{R}(X, \mathbb{F})$ is dense in $\mathcal{C}(X, \mathbb{F})$. Consequently, Swan's theorem is applicable.

Denote by $K_{\mathbb{F} \text {-alg }}(X), K_{\mathbb{F}-\mathcal{X}}(X), K_{\mathbb{F} \text {-str }}(X)$ and $K_{\mathbb{F}}(X)$ the Grothendieck group of the commutative monoids $\operatorname{VB}_{\mathbb{F} \text {-alg }}(X), \operatorname{VB}_{\mathbb{F}-\mathcal{X}}(X), \operatorname{VB}_{\mathbb{F} \text {-str }}(X)$ and $\mathrm{VB}_{\mathbb{F}}(X)$. Note that $K_{\mathbb{F} \text {-alg }}(X)=K_{\mathbb{F}-\{X\}}(X)$.

As usual, for any ring $R$, the Grothendieck group of the commutative monoid $P(R)$ will be denoted by $K_{0}(R)$.

Corollary 3.11. The commutative diagram in Theorem 3.10 gives rise to a commutative diagram

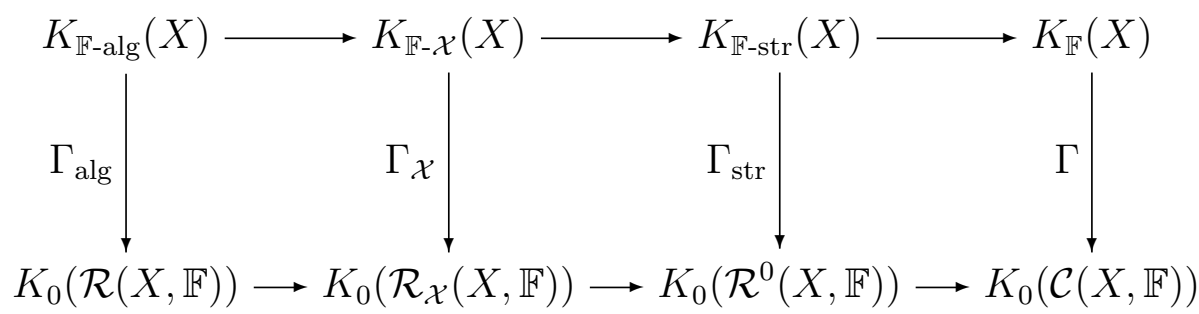

in which the vertical homomorphisms are all isomorphisms. Furthermore, if the variety $X$ is compact, then the horizontal homomorphisms are all monomorphisms.

Proof. It suffices to make use of Theorem 3.10 .

Corollary 3.12. Assume that the variety $X$ is compact. For a stratified-algebraic $\mathbb{F}$-vector bundle $\xi$ on $X$, the following conditions are equivalent:

(a) $\xi$ is isomorphic in the category of stratified-algebraic $\mathbb{F}$-vector bundles on $X$ to an $\mathcal{X}$-algebraic $\mathbb{F}$-vector bundle on $X$.

(b) $\xi$ is isomorphic in the category of topological $\mathbb{F}$-vector bundles on $X$ to an $\mathcal{X}$ algebraic $\mathbb{F}$-vector bundle on $X$.

(c) The class of $\xi$ in $K_{\mathbb{F}-\text { str }}(X)$ belongs to the image of the canonical homomorphism $K_{\mathbb{F}-\mathcal{X}}(X) \rightarrow K_{\mathbb{F}-\text { str }}(X)$.

(d) The class of $\xi$ in $K_{\mathbb{F}}(X)$ belongs to the image of the canonical homomorphism $K_{\mathbb{F}-\mathcal{X}}(X) \rightarrow K_{\mathbb{F}}(X)$.

Proof. In view of Theorem 3.10 and Corollary 3.11, it suffices to apply Swan's theorem [45, Theorem 2.2].

In the same way, we obtain the next two corollaries, which are recorded for the sake of completeness.

Corollary 3.13. Assume that the variety $X$ is compact. For a topological $\mathbb{F}$-vector bundle $\xi$ on $X$, the following conditions are equivalent:

(a) $\xi$ is isomorphic in the category of topological $\mathbb{F}$-vector bundles on $X$ to an $\mathcal{X}$ algebraic $\mathbb{F}$-vector bundle on $X$. 
(b) The class of $\xi$ in $K_{\mathbb{F}}(X)$ belongs to the image of the canonical homomorphism $K_{\mathbb{F}-\mathcal{X}}(X) \rightarrow K_{\mathbb{F}}(X)$.

Corollary 3.14. Assume that the variety $X$ is compact. For a topological $\mathbb{F}$-vector bundle $\xi$ on $X$, the following conditions are equivalent:

(a) $\xi$ is isomorphic in the category of topological $\mathbb{F}$-vector bundles on $X$ to a stratifiedalgebraic $\mathbb{F}$-vector bundle on $X$.

(b) The class of $\xi$ in $K_{\mathbb{F}}(X)$ belongs to the image of the canonical homomorphism $K_{\mathbb{F} \text {-str }}(X) \rightarrow K_{\mathbb{F}}(X)$.

Now we are in a position to prove two results announced in Section 1.

Proof of Theorem 1.5. Recall that the group $K_{\mathbb{F}}(X)$ is finitely generated (cf. [30, Exercise III.7.5] or the spectral sequence in [4, 21]). According to Corollary 3.11, the group $K_{\mathbb{F} \text {-str }}(X)$ also is finitely generated. Hence there exist stratified-algebraic $\mathbb{F}$-vector bundles $\xi_{1}, \ldots, \xi_{r}$ on $X$ whose classes in $K_{\mathbb{F} \text {-str }}(X)$ generate this group. We can find a stratification $\mathcal{S}$ of $X$ such that each $\xi_{i}$ is $\mathcal{S}$-algebraic for $1 \leq i \leq r$. Consequently, the canonical homomorphism $K_{\mathbb{F}-\mathcal{S}}(X) \rightarrow K_{\mathbb{F} \text {-str }}(X)$ is surjective. The proof is complete in view of Corollary 3.12 .

Proof of Theorem 1.6. As in the proof of Theorem 1.5, we obtain stratified-algebraic $\mathbb{F}$ vector bundles $\xi_{1}, \ldots, \xi_{r}$ on $X$ whose classes in $K_{\mathbb{F}-\text { str }}(X)$ generate this group. According to Proposition 3.4, each $\xi_{i}$ is of the form $\xi_{i}=f_{i}^{*} \gamma_{K_{i}}\left(\mathbb{F}^{n_{i}}\right)$ for some multi-Grassmannian $\mathbb{G}_{K_{i}}\left(\mathbb{F}^{n_{i}}\right)$ and stratified-regular map $f_{i}: X \rightarrow \mathbb{G}_{K_{i}}\left(\mathbb{F}^{n_{i}}\right)$. Set

$$
f:=\left(f_{1}, \ldots, f_{r}\right): X \rightarrow G:=\mathbb{G}_{K_{1}}\left(\mathbb{F}^{n_{1}}\right) \times \cdots \times \mathbb{G}_{K_{r}}\left(\mathbb{F}^{n_{r}}\right) .
$$

By Hironaka's theorem on resolution of singularities [26, 31], there exists a birational multiblowup $\rho: \tilde{X} \rightarrow X$ such that the variety $\tilde{X}$ is nonsingular. The composite map $f \circ \rho: \tilde{X} \rightarrow G$ is stratified-regular, and hence, in view of Proposition 2.2, it is continuous rational. Now, Hironaka's theorem on resolution of points of indeterminacy [27, 31] implies the existence of a birational multiblowup $\sigma: X^{\prime} \rightarrow \tilde{X}$ such that the composite map $f \circ \rho \circ \sigma: X^{\prime} \rightarrow G$ is regular. The variety $X^{\prime}$ is nonsingular and $\pi:=\rho \circ \sigma: X^{\prime} \rightarrow X$ is a birational multiblowup. Since the composite map $f_{i} \circ \pi$ is regular, the pullback

$$
\pi^{*} \xi_{i}=\pi^{*}\left(f_{i}^{*} \gamma_{K_{i}}\left(\mathbb{F}^{n_{i}}\right)\right)=\left(f_{i} \circ \pi\right)^{*} \gamma_{K_{i}}\left(\mathbb{F}^{n_{i}}\right)
$$

is an algebraic $\mathbb{F}$-vector bundle on $X^{\prime}$ for $1 \leq i \leq r$. Since the classes of $\xi_{1}, \ldots, \xi_{r}$ generate the group $K_{\mathbb{F} \text {-str }}(X)$, it follows that the image of the homomorphism

$$
\pi^{*}: K_{\mathbb{F}-\operatorname{str}}(X) \rightarrow K_{\mathbb{F}-\text { str }}\left(X^{\prime}\right)
$$

induced by $\pi$ is contained in the image of the canonical homomorphism

$$
K_{\mathbb{F} \text {-alg }}\left(X^{\prime}\right)=K_{\mathbb{F}-\left\{X^{\prime}\right\}}\left(X^{\prime}\right) \rightarrow K_{\mathbb{F} \text {-str }}\left(X^{\prime}\right) .
$$

Hence, in view of Corollary 3.11 (with $X=X^{\prime}$ ), for any stratified-algebraic $\mathbb{F}$-vector bundle $\xi$ on $X$, the pullback $\pi^{*} \xi$ is isomorphic in the category of stratified-algebraic $\mathbb{F}$ vector bundles on $X^{\prime}$ to an algebraic $\mathbb{F}$-vector bundle on $X^{\prime}$. This proves Theorem 1.6 . 
We conclude this section by showing that the categories of vector bundles considered here are closed under standard operations. This is made precise in Proposition 3.15 and its proof. In fact it was already done for the direct sum $\oplus$ when the monoids $\operatorname{VB}_{\mathbb{F}-\mathcal{X}}(X)$ and $\operatorname{VB}_{\mathbb{F} \text {-str }}(X)$ were introduced.

Proposition 3.15. Let $\xi$ and $\eta$ be $\mathcal{X}$-algebraic $\mathbb{F}$-vector bundles on $X$. Then the $\mathbb{F}$-vector bundles $\xi \oplus \eta, \operatorname{Hom}(\xi, \eta)$ and $\xi^{\vee}$ (dual bundle) are $\mathcal{X}$-algebraic. If $\mathbb{F}=\mathbb{R}$ or $\mathbb{F}=\mathbb{C}$, then the $\mathbb{F}$-vector bundles $\xi \otimes \eta$ and $\bigwedge^{k} \xi$ (kth exterior power) are $\mathcal{X}$-algebraic. Furthermore, the analogous statements hold true for stratified-algebraic $\mathbb{F}$-vector bundles.

Proof. Suppose that $\xi$ is an $\mathcal{X}$-algebraic subbundle of $\varepsilon_{X}^{n}(\mathbb{F})$ and $\eta$ is an $\mathcal{X}$-algebraic subbundle of $\varepsilon_{X}^{m}(\mathbb{F})$. We already know that $\xi \oplus \eta$ is regarded as a subbundle of

$$
\varepsilon_{X}^{n}(\mathbb{F}) \oplus \varepsilon_{X}^{m}(\mathbb{F}) \cong \varepsilon_{X}^{n+m}(\mathbb{F})
$$

Since $\xi \oplus \xi^{\perp}=\varepsilon_{X}^{n}(\mathbb{F})$ and $\eta \oplus \eta^{\perp}=\varepsilon_{X}^{m}(\mathbb{F})$, (cf. Proposition 3.7), $\operatorname{Hom}(\xi, \eta)$ can be regarded as a subbundle of $\operatorname{Hom}\left(\varepsilon_{X}^{n}(\mathbb{F}), \varepsilon_{X}^{m}(\mathbb{F})\right) \cong \varepsilon_{X}^{n m}(\mathbb{F})$. By dualizing the orthogonal projection $\varepsilon_{X}^{n}(\mathbb{F}) \rightarrow \xi$, we regard $\xi^{\vee}$ as a subbundle of $\varepsilon_{X}^{n}(\mathbb{F})^{\vee} \cong \varepsilon_{X}^{n}(\mathbb{F})$. If $\mathbb{F}=\mathbb{R}$ or $\mathbb{F}=\mathbb{C}$, then $\xi \otimes \eta$ can be regarded as a subbundle of $\varepsilon_{X}^{n}(\mathbb{F}) \otimes \varepsilon_{X}^{m}(\mathbb{F}) \cong \varepsilon_{X}^{n m}(\mathbb{F})$, and $\bigwedge^{k} \xi$ can be regarded as a subbundle of $\bigwedge^{k} \varepsilon_{X}^{n}(\mathbb{F}) \cong \varepsilon_{X}^{q}(\mathbb{F})$, where $q=\left(\begin{array}{l}n \\ k\end{array}\right)$ is the binomial coefficient. After these identifications, each of the vector bundles under consideration becomes an $\mathcal{X}$-algebraic subbundle of $\varepsilon_{X}^{p}(\mathbb{F})$ for an appropriate $p$. The same argument works for stratified-algebraic vector bundles.

\section{Approximation by stratified-regular maps}

As in Section 3, for any real algebraic variety $X$ with stratification $\mathcal{X}$, and any real algebraic variety $Y$, we have the following inclusions:

$$
\mathcal{R}(X, Y) \subseteq \mathcal{R}_{\mathcal{X}}(X, Y) \subseteq \mathcal{R}^{0}(X, Y) \subseteq \mathcal{C}(X, Y)
$$

A challenging problem is to find a useful description of the closure of each of the sets $\mathcal{R}(X, Y), \mathcal{R}_{\mathcal{X}}(X, Y)$ and $\mathcal{R}^{0}(X, Y)$ in the space $\mathcal{C}(X, Y)$, endowed with the compactopen topology. In other words, the problem is to find a characterization of these maps in $\mathcal{C}(X, Y)$ that can be approximated by either regular or $\mathcal{X}$-regular or stratified-regular maps. Approximation by regular maps is investigated in [9, 10, 12, 13, 16, 18. As demonstrated in [33, 35], stratified-regular maps are much more flexible than regular maps. In the present section, we prove approximation theorems for maps with values in multiGrassmannians. It is important for applications to obtain results in which approximating maps satisfy some extra conditions.

We first recall a key extension result due to Kollár and Nowak [32, Theorem 9, Proposition 10].

Theorem 4.1 ([32]). Let $X$ be a real algebraic variety. Let $A$ and $B$ be Zariski closed subvarieties of $X$ with $B \subseteq A$. For any stratified-regular function $f: A \rightarrow \mathbb{R}$ whose restriction $\left.f\right|_{A \backslash B}$ is a regular function, there exists a stratified-regular function $F: X \rightarrow \mathbb{R}$ such that $\left.F\right|_{A}=f$ and $\left.F\right|_{X \backslash B}$ is a regular function.

It should be mentioned that in [32], Theorem 4.1 is stated in terms of hereditarily rational functions (cf. Remark 2.3). 
The following terminology will be convenient. We say that a topological $\mathbb{F}$-vector bundle on a real algebraic variety $X$ admits an algebraic (resp. $\mathcal{X}$-algebraic, stratifiedalgebraic) structure if it is topologically isomorphic to an algebraic (resp. $\mathcal{X}$-algebraic, stratified-algebraic) $\mathbb{F}$-vector bundle on $X$. If $Z$ is a Zariski closed subvariety of $X$, we say that a multiblowup $\pi: X^{\prime} \rightarrow X$ of $X$ is over $Z$ if the restriction $\pi_{Z}: X^{\prime} \backslash \pi^{-1}(Z) \rightarrow X \backslash Z$ of $\pi$ is a biregular isomorphism.

Notation 4.2. In the remainder of this section, $X$ denotes a compact real algebraic variety, and $A$ is a Zariski closed subvariety of $X$. Moreover, $\mathbb{G}_{K}\left(\mathbb{F}^{n}\right)$ is the $(K, n)$-multiGrassmannian and $\gamma_{K}\left(\mathbb{F}^{n}\right)$ is the tautological $\mathbb{F}$-vector bundle on $\mathbb{G}_{K}\left(\mathbb{F}^{n}\right)$, for some fixed $(K, n)$ (cf. Section 2).

Our basic approximation result is the following.

Lemma 4.3. Let $B$ be a Zariski closed subvariety of $X$ with $B \subseteq A$. Let $f: X \rightarrow \mathbb{R}$ be a continuous function such that $\left.f\right|_{A}$ is a stratified-regular function and $\left.f\right|_{A \backslash B}$ is a regular function. For every $\varepsilon>0$, there exists a stratified-regular function $g: X \rightarrow \mathbb{R}$ such that $\left.g\right|_{A}=\left.f\right|_{A}$, the restriction $\left.g\right|_{X \backslash B}$ is a regular function, and

$$
|g(x)-f(x)|<\varepsilon \quad \text { for all } x \text { in } X .
$$

Proof. According to Theorem 4.1, there exists a stratified-regular function $h: X \rightarrow \mathbb{R}$ such that $\left.h\right|_{A}=\left.f\right|_{A}$ and the restriction $\left.h\right|_{X \backslash B}$ is a regular function. The function $\varphi:=f-h$ is continuous and $\left.\varphi\right|_{A}=0$. Hence, by [15, Lemma 2.1], one can find a regular function $\psi: X \rightarrow \mathbb{R}$ satisfying $\left.\psi\right|_{A}=0$ and

$$
|\varphi(x)-\psi(x)|<\varepsilon \quad \text { for all } x \text { in } X \text {. }
$$

The function $g:=h+\psi$ has the required properties.

For any stratification $\mathcal{X}$ of $X$ and any Zariski closed subvariety $B$ of $X$, we set

$$
\mathcal{X} \cap B:=\{S \cap B \mid S \in \mathcal{X}\} \quad \text { and } \quad \mathcal{X} \backslash B:=\{S \backslash B \mid S \in \mathcal{X}\} .
$$

Then

$$
\mathcal{X}_{B}:=(\mathcal{X} \cap B) \cup\{\mathcal{X} \backslash B\}
$$

is a stratification of $X$. Obviously $\mathcal{X}_{B} \cap A$ is a stratification of $A$.

Theorem 4.4. Let $\mathcal{X}$ be a stratification of $X$ and let $B$ be a Zariski closed subvariety of $X$. Let $f: X \rightarrow \mathbb{G}_{K}\left(\mathbb{F}^{n}\right)$ be a continuous map whose restriction $\left.f\right|_{A}$ is $\left(\mathcal{X}_{B} \cap A\right)$-regular. If $B \subseteq A$ and $A \backslash B$ is a stratum in $\mathcal{X}_{B}$, then the following conditions are equivalent:

(a) Each neighborhood of $f$ in $\mathcal{C}\left(X, \mathbb{G}_{K}\left(\mathbb{F}^{n}\right)\right)$ contains an $\mathcal{X}_{B}$-regular map

$$
g: X \rightarrow \mathbb{G}_{K}\left(\mathbb{F}^{n}\right)
$$

with $\left.g\right|_{A}=\left.f\right|_{A}$.

(b) The map $f$ is homotopic to an $\mathcal{X}_{B}$-regular map $h: X \rightarrow \mathbb{G}_{K}\left(\mathbb{F}^{n}\right)$ with $\left.h\right|_{A}=\left.f\right|_{A}$.

(c) The pullback $\mathbb{F}$-vector bundle $f^{*} \gamma_{K}\left(\mathbb{F}^{n}\right)$ on $X$ admits an $\mathcal{X}_{B}$-algebraic structure. 
Proof. It is a standard fact that (a) implies (b). If (b) holds, then the $\mathcal{X}_{B}$-algebraic $\mathbb{F}$ vector bundle $h^{*} \gamma_{K}\left(\mathbb{F}^{n}\right)$ is topologically isomorphic to $f^{*} \gamma_{K}\left(\mathbb{F}^{n}\right)$, and hence (c) holds. It suffices to prove that (c) implies (a).

Suppose that (c) holds. In order to simplify notation, we set $G=\mathbb{G}_{K}\left(\mathbb{F}^{n}\right), \gamma=\gamma_{K}\left(F^{n}\right)$, $\mathcal{A}=\mathcal{X}_{B} \cap A$, and $\varepsilon_{X}^{l}=\varepsilon_{X}^{l}\left(\mathbb{F}^{n}\right)$ for any nonnegative integer $l$. Condition (c) implies the existence of a topological isomorphism $\varphi: \xi \rightarrow f^{*} \gamma$, where $\xi$ is an $\mathcal{X}_{B}$-algebraic $\mathbb{F}$-vector subbundle of $\varepsilon_{X}^{k}$ for some $k$. Regarding $f^{*} \gamma$ as an $\mathbb{F}$-vector subbundle of $\varepsilon_{X}^{n}$, we get a continuous section $w: X \rightarrow \operatorname{Hom}\left(\xi, \varepsilon_{X}^{n}\right)$, defined by $w(x)(e)=\varphi(e)$ for all points $x$ in $X$ and all vectors $e$ in the fiber $E(\xi)_{x}$. In particular, $w(x): E(\xi)_{x} \rightarrow\{x\} \times \mathbb{F}^{n}$ is an injective $\mathbb{F}$-linear transformation satisfying

$$
w(x)\left(E(\xi)_{x}\right)=\{x\} \times f(x) .
$$

Making use of the equalities $\varepsilon_{X}^{k}=\xi \oplus \xi^{\perp}$ and $\varepsilon_{X}^{n}=f^{*} \gamma \oplus f^{*} \gamma^{\perp}$, we find a continuous section $\bar{w}: X \rightarrow \operatorname{Hom}\left(\varepsilon_{X}^{k}, \varepsilon_{X}^{n}\right)$ satisfying $\bar{w}(x)\left(E\left(\varepsilon_{X}^{k}\right)_{x}\right) \subseteq E\left(f^{*} \gamma\right)_{x}$ and $\left.\bar{w}(x)\right|_{E(\xi)_{x}}=w(x)$ for all $x$ in $X$. Since $X$ is compact and the $\mathbb{F}$-vector bundle $\operatorname{Hom}\left(\varepsilon_{X}^{k}, \varepsilon_{X}^{n}\right)$ can be identified with $\varepsilon_{X}^{k n}$, the Weierstrass approximation theorem implies the existence of an algebraic section $v: X \rightarrow \operatorname{Hom}\left(\varepsilon_{X}^{k}, \varepsilon_{X}^{n}\right)$ arbitrarily close to $\bar{w}$ in the compact-open topology. If $\rho: \varepsilon_{X}^{n} \rightarrow f^{*} \gamma$ is the orthogonal projection and $\iota: f^{*} \gamma \hookrightarrow \varepsilon_{X}^{n}$ is the inclusion morphism, then $\bar{v}:=\iota \circ \rho \circ v: X \rightarrow \operatorname{Hom}\left(\varepsilon_{X}^{k}, \varepsilon_{X}^{n}\right)$ is a continuous section close to $\bar{w}$. If $v$ is sufficiently close to $\bar{w}$, then the $\mathbb{F}$-linear transformation $\bar{v}(x): E(\xi)_{x} \rightarrow\{x\} \times \mathbb{F}^{n}$ is injective and

$$
\bar{v}(x)\left(E(\xi)_{x}\right)=\{x\} \times f(x)
$$

for all $x$ in $X$. Since the map $\left.f\right|_{A}$ is $\mathcal{A}$-regular, the $\mathbb{F}$-vector bundle $\left(\left.f\right|_{A}\right)^{*} \gamma=\left.\left(f^{*} \gamma\right)\right|_{A}$ on $A$ is $\mathcal{A}$-algebraic. Hence, in view of Proposition 3.7, the restriction $\rho_{A}:\left.\left.\varepsilon_{X}^{n}\right|_{A} \rightarrow\left(f^{*} \gamma\right)\right|_{A}$ of $\rho$ is an $\mathcal{A}$-algebraic morphism. Consequently, the restriction $\left.\bar{v}\right|_{A}: A \rightarrow \operatorname{Hom}\left(\varepsilon_{X}^{k}, \varepsilon_{X}^{n}\right)$ of $\bar{v}$ is an $\mathcal{A}$-algebraic section. By Lemma 4.3 , there exists a continuous section

$$
u: X \rightarrow \operatorname{Hom}\left(\varepsilon_{X}^{k}, \varepsilon_{X}^{n}\right) \cong \varepsilon_{X}^{k n}
$$

such that $\left.u\right|_{A}=\left.\bar{v}\right|_{A}$, the restriction $\left.u\right|_{X \backslash B}$ is an algebraic section, and $u$ is close to $\bar{v}$. If $u$ is sufficiently close to $\bar{v}$, then the $\mathbb{F}$-linear transformation $u(x): E(\xi)_{x} \rightarrow\{x\} \times \mathbb{F}^{n}$ is injective for all $x$ in $X$. Now, the map $g: X \rightarrow G$, defined by

$$
u(x)\left(E(\xi)_{x}\right)=\{x\} \times g(x)
$$

for all $x$ in $X$, is continuous and close to $f$, and $\left.g\right|_{A}=\left.f\right|_{A}$. If $T$ is a stratum in $\mathcal{X}_{B}$, then $\left.u\right|_{T}$ is an algebraic section and $\left.\xi\right|_{T}$ is an algebraic $\mathbb{F}$-vector subbundle of $\left.\varepsilon_{X}^{k}\right|_{T}$, and hence the map $\left.g\right|_{T}$ is regular (cf. [9, Proposition 3.4.7]). Consequently, $g$ is an $\mathcal{X}_{B}$-regular map. Thus, (c) implies (a).

It is worthwhile to state several consequences of Theorem 4.4 .

Corollary 4.5. Let $f: X \rightarrow \mathbb{G}_{K}\left(\mathbb{F}^{n}\right)$ be a continuous map whose restriction $\left.f\right|_{A}$ is regular. Then the following conditions are equivalent:

(a) Each neighborhood of $f$ in $\mathcal{C}\left(X, \mathbb{G}_{K}\left(\mathbb{F}^{n}\right)\right)$ contains a regular map $g: X \rightarrow \mathbb{G}_{K}\left(\mathbb{F}^{n}\right)$ with $\left.g\right|_{A}=\left.f\right|_{A}$.

(b) The map $f$ is homotopic to a regular map $h: X \rightarrow \mathbb{G}_{K}\left(\mathbb{F}^{n}\right)$ with $\left.h\right|_{A}=\left.f\right|_{A}$. 
(c) The pullback $\mathbb{F}$-vector bundle $f^{*} \gamma_{K}\left(\mathbb{F}^{n}\right)$ on $X$ admits an algebraic structure.

Proof. If $\mathcal{X}=\{X\}$ and $B=\varnothing$, then $\mathcal{X}_{B}=\{X\}$ and $\mathcal{X}_{B} \cap A=\{A\}$. Hence, it suffices to apply Theorem 4.4 .

Corollary 4.5 with $A=\varnothing$ is well known, cf. [10] or [9, Theorem 13.3.1].

Corollary 4.6. Let $\mathcal{X}$ be a stratification of $X$ such that $\mathcal{A}:=\{S \in \mathcal{X} \mid S \subseteq A\}$ is a stratification of $A$. Let $f: X \rightarrow \mathbb{G}_{K}\left(\mathbb{F}^{n}\right)$ be a continuous map whose restriction $\left.f\right|_{A}$ is $\mathcal{A}$-regular. Then the following conditions are equivalent:

(a) Each neighborhood of $f$ in $\mathcal{C}\left(X, \mathbb{G}_{K}\left(\mathbb{F}^{n}\right)\right)$ contains an $\mathcal{X}$-regular map $g: X \rightarrow \mathbb{G}_{K}\left(\mathbb{F}^{n}\right)$ with $\left.g\right|_{A}=\left.f\right|_{A}$.

(b) The map $f$ is homotopic to an $\mathcal{X}$-regular map $h: X \rightarrow \mathbb{G}_{K}\left(\mathbb{F}^{n}\right)$ with $\left.h\right|_{A}=\left.f\right|_{A}$.

(c) The pullback $\mathbb{F}$-vector bundle $f^{*} \gamma_{K}\left(\mathbb{F}^{n}\right)$ on $X$ admits an $\mathcal{X}$-algebraic structure.

Proof. If $B=A$, then $\mathcal{X}_{B}=\mathcal{X}$ and $\mathcal{X}_{B} \cap A=\mathcal{A}$, and hence it suffices to apply Theorem 4.4.

Corollary 4.7. Let $\mathcal{X}$ be a stratification of $X$. For a continuous map $f: X \rightarrow \mathbb{G}_{K}\left(\mathbb{F}^{n}\right)$, the following conditions are equivalent:

(a) The map $f$ can be approximated by $\mathcal{X}$-regular maps.

(b) The map $f$ is homotopic to an $\mathcal{X}$-regular map.

(c) The pullback $\mathbb{F}$-vector bundle $f^{*} \gamma_{K}\left(\mathbb{F}^{n}\right)$ on $X$ admits an $\mathcal{X}$-algebraic structure.

Proof. This is Corollary 4.6 with $A=\varnothing$.

Corollary 4.7 makes it possible to interpret Theorems 1.5 and 1.6 as approximation results.

Theorem 4.8. There exists a stratification $\mathcal{S}$ of $X$ such that any stratified-regular map from $X$ into $\mathbb{G}_{K}\left(\mathbb{F}^{n}\right)$ can be approximated by $\mathcal{S}$-regular maps.

Proof. Let $\mathcal{S}$ be a stratification of $X$ as in Theorem 1.5. By Proposition 2.6, if

$$
f: X \rightarrow \mathbb{G}_{K}\left(\mathbb{F}^{n}\right)
$$

is a stratified-regular map, then the $\mathbb{F}$-vector bundle $f^{*} \gamma_{K}\left(\mathbb{F}^{n}\right)$ on $X$ is stratified-algebraic. It follows that $f^{*} \gamma_{K}\left(\mathbb{F}^{n}\right)$ admits an $\mathcal{S}$-algebraic structure. According to Corollary 4.7, $f$ can be approximated by $\mathcal{S}$-regular maps.

Theorem 4.9. There exists a birational multiblowup $\pi: X^{\prime} \rightarrow X$, with $X^{\prime}$ a nonsingular variety, such that for any stratified-regular map $f: X \rightarrow \mathbb{G}_{K}\left(\mathbb{F}^{n}\right)$, the composite map $f \circ \pi: X^{\prime} \rightarrow \mathbb{G}_{K}\left(\mathbb{F}^{n}\right)$ can be approximated by regular maps.

Proof. Let $\pi: X^{\prime} \rightarrow X$ be a birational multiblowup as in Theorem 1.6. By Proposition 2.6, if $f: X \rightarrow \mathbb{G}_{K}\left(\mathbb{F}^{n}\right)$ is a stratified-regular map, the $\mathbb{F}$-vector bundle $f^{*} \gamma_{K}\left(\mathbb{F}^{n}\right)$ on $X$ is stratified-algebraic. It follows that the $\mathbb{F}$-vector bundle

$$
\pi^{*}\left(f^{*} \gamma_{K}\left(\mathbb{F}^{n}\right)\right)=(f \circ \pi)^{*} \gamma_{K}\left(\mathbb{F}^{n}\right)
$$

on $X^{\prime}$ admits an algebraic-structure. According to Corollary 4.7 (with $X=X^{\prime}$ and $\left.\mathcal{X}=\left\{X^{\prime}\right\}\right)$, the map $f \circ \pi: X^{\prime} \rightarrow \mathbb{G}_{K}\left(\mathbb{F}^{n}\right)$ can be approximated by regular maps. 
It follows from Corollary 4.7 and Propositions 2.6 and 3.4 that Theorem 4.9 is equivalent to Theorem 1.6.

Theorem 4.10. Let $f: X \rightarrow \mathbb{G}_{K}\left(\mathbb{F}^{n}\right)$ be a continuous map whose restriction $\left.f\right|_{A}$ is stratified-regular. Then the following conditions are equivalent:

(a) Each neighborhood of $f$ in $\mathcal{C}\left(X, \mathbb{G}_{K}\left(\mathbb{F}^{n}\right)\right)$ contains a stratified-regular map

$$
g: X \rightarrow \mathbb{G}_{K}\left(\mathbb{F}^{n}\right)
$$

with $\left.g\right|_{A}=\left.f\right|_{A}$.

(b) The map $f$ is homotopic to a stratified-regular map $h: X \rightarrow \mathbb{G}_{K}\left(\mathbb{F}^{n}\right)$ with $\left.h\right|_{A}=\left.f\right|_{A}$.

(c) The pullback $\mathbb{F}$-vector bundle $f^{*} \gamma_{K}\left(\mathbb{F}^{n}\right)$ on $X$ admits a stratified-algebraic structure.

Proof. As in the case of Theorem 4.4, it suffices to prove that (c) implies (a).

Suppose that (c) holds. Let $\mathcal{T}$ be a stratification of $X$ such that the $\mathbb{F}$-vector bundle $f^{*} \gamma_{K}\left(\mathbb{F}^{n}\right)$ admits a $\mathcal{T}$-algebraic structure, and let $\mathcal{P}$ be a stratification of $A$ such that the map $\left.f\right|_{A}$ is $\mathcal{P}$-regular. The collection

$$
\mathcal{X}:=\{P \cap T \mid P \in \mathcal{P}, T \in \mathcal{T}\} \cup\{T \cap(X \backslash A) \mid T \in \mathcal{T}\}
$$

is a stratification of $X$, and $\mathcal{A}:=\{S \in \mathcal{X} \mid S \subseteq A\}$ is a stratification of $A$. By construction, $\mathcal{X}$ is a refinement of $\mathcal{T}$, and $\mathcal{A}$ is a refinement of $\mathcal{P}$. Consequently, the map $\left.f\right|_{A}$ is $\mathcal{A}$ regular, and the $\mathbb{F}$-vector bundle $f^{*} \gamma_{K}\left(\mathbb{F}^{n}\right)$ on $X$ admits an $\mathcal{X}$-algebraic structure. The proof is complete in view of Corollary 4.6 .

In the results above, approximation by $\mathcal{X}$-regular or stratified-regular maps is equivalent to certain conditions on pullbacks of the tautological vector bundle. It is often convenient (see Sections 5 and 6) to have these conditions expressed in terms involving multiblowups.

Proposition 4.11. Let $f: X \rightarrow \mathbb{G}_{K}\left(\mathbb{F}^{n}\right)$ be a continuous map whose restriction $\left.f\right|_{A}$ is stratified-regular. Assume the existence of a multiblowup $\pi: X^{\prime} \rightarrow X$ over $A$ such that the pullback $\mathbb{F}$-vector bundle $(f \circ \pi)^{*} \gamma_{K}\left(\mathbb{F}^{n}\right)$ on $X^{\prime}$ admits an algebraic structure. Then each neighborhood of $f$ in $\mathcal{C}\left(X, \mathbb{G}_{K}\left(\mathbb{F}^{n}\right)\right)$ contains a stratified-regular map $g: X \rightarrow \mathbb{G}_{K}\left(\mathbb{F}^{n}\right)$ such that $\left.g\right|_{A}=\left.f\right|_{A}$ and the restriction $\left.g\right|_{X \backslash A}$ is a regular map. In particular, $f$ is homotopic to a stratified-regular map $h: X \rightarrow \mathbb{G}_{K}\left(\mathbb{F}^{n}\right)$ such that $\left.h\right|_{A}=\left.f\right|_{A}$ and the restriction $\left.h\right|_{X \backslash A}$ is a regular map.

Proof. The map $\left.f\right|_{A}$ is $\mathcal{A}$-regular for some stratification $\mathcal{A}$ of $A$. The collection $\mathcal{A}^{\prime}:=\left\{\pi^{-1}(S) \mid S \in \mathcal{A}\right\}$ is a stratification of $A^{\prime}:=\pi^{-1}(A)$, whereas $\mathcal{X}^{\prime}:=\mathcal{A}^{\prime} \cup\left\{X^{\prime} \backslash A^{\prime}\right\}$ is a stratification of $X^{\prime}$. Since the map $f \circ \pi: X^{\prime} \rightarrow \mathbb{G}_{K}\left(\mathbb{F}^{n}\right)$ is continuous and its restriction $\left.(f \circ \pi)\right|_{A^{\prime}}$ is $\mathcal{A}^{\prime}$-regular, according to Corollary 4.6 (with $X=X^{\prime}, A=A^{\prime}, \mathcal{X}=\mathcal{X}^{\prime}$, $\left.\mathcal{A}=\mathcal{A}^{\prime}, f=f \circ \pi\right)$, there exists an $\mathcal{X}^{\prime}$-regular map $\varphi: X^{\prime} \rightarrow \mathbb{G}_{K}\left(\mathbb{F}^{n}\right)$ that is arbitrarily close to $f \circ \pi$ and satisfies $\left.\varphi\right|_{A^{\prime}}=\left.(f \circ \pi)\right|_{A^{\prime}}$. In particular, the restriction $\left.\varphi\right|_{X^{\prime} \backslash A^{\prime}}$ is a regular map. By assumption, the restriction $\pi_{A}: X^{\prime} \backslash A^{\prime} \rightarrow X \backslash A$ of $\pi$ is a biregular isomorphism. Since the map $\pi$ is proper, the map $g: X \rightarrow \mathbb{G}_{K}\left(\mathbb{F}^{n}\right)$, defined by

$$
g(x)= \begin{cases}\varphi\left(\pi_{A}^{-1}(x)\right) & \text { for } x \text { in } X \backslash A \\ f(x) & \text { for } x \text { in } A,\end{cases}
$$

is continuous. Moreover, $g$ is close to $f,\left.g\right|_{A}=\left.f\right|_{A}$, and the restriction $\left.g\right|_{X \backslash A}$ is a regular map. If $g$ is sufficiently close to $f$, then $g$ is homotopic to $f$, and hence the existence of $h$ follows. 
Corollary 4.12. Let $f: X \rightarrow \mathbb{G}_{K}\left(\mathbb{F}^{n}\right)$ be a continuous map whose restriction $\left.f\right|_{A}$ is stratified-regular. If the variety $X \backslash A$ is nonsingular, then the following conditions are equivalent:

(a) Each neighborhood of $f$ in $\mathcal{C}\left(X, \mathbb{G}_{K}\left(\mathbb{F}^{n}\right)\right)$ contains a stratified-regular map

$$
g: X \rightarrow \mathbb{G}_{K}\left(\mathbb{F}^{n}\right)
$$

such that $\left.g\right|_{A}=\left.f\right|_{A}$ and the restriction $\left.g\right|_{X \backslash A}$ is a regular map.

(b) The map $f$ is homotopic to a stratified-regular map $h: X \rightarrow \mathbb{G}_{K}\left(\mathbb{F}^{n}\right)$ such that $\left.h\right|_{A}=\left.f\right|_{A}$ and the restriction $\left.h\right|_{X \backslash A}$ is a regular map.

(c) There exists a multiblowup $\pi: X^{\prime} \rightarrow X$ over $A$ such that $X^{\prime}$ is a nonsingular variety and the pullback $\mathbb{F}$-vector bundle $(f \circ \pi)^{*} \gamma_{K}\left(\mathbb{F}^{n}\right)$ on $X^{\prime}$ admits an algebraic structure.

Proof. It is a standard fact that (a) implies (b). In view of Proposition 4.11, (c) implies (a). It remains to prove that (b) implies (c).

Suppose that (b) holds. Since the variety $X \backslash A$ is nonsingular, Hironaka's theorem on resolution of singularities [26, 31] implies the existence of a multiblowup $\rho: \tilde{X} \rightarrow X$ over $A$ such that $\tilde{X}$ is a nonsingular variety and the set $\tilde{X} \backslash \rho^{-1}(A)$ is Zariski dense in $\tilde{X}$. The map $h \circ \rho: \tilde{X} \rightarrow \mathbb{G}_{K}\left(\mathbb{F}^{n}\right)$ is continuous and its restriction to $\tilde{X} \backslash \rho^{-1}(A)$ is a regular map. Hence, according to Hironaka's theorem on resolution of points of indeterminacy [26, 31], there exists a multiblowup $\sigma: X^{\prime} \rightarrow \tilde{X}$ over $\rho^{-1}(A)$ such that the map $h \circ \pi: X^{\prime} \rightarrow \mathbb{G}_{K}\left(\mathbb{F}^{n}\right)$ is regular, where $\pi:=\rho \circ \sigma$. Consequently, $(h \circ \pi)^{*} \gamma_{K}\left(\mathbb{F}^{n}\right)$ is an algebraic $\mathbb{F}$-vector bundle on $X^{\prime}$. The variety $X^{\prime}$ is nonsingular, and $\pi: X^{\prime} \rightarrow X$ is a multiblowup over $A$. Since the maps $h \circ \pi$ and $f \circ \pi$ are homotopic, the $\mathbb{F}$-vector bundles $(h \circ \pi)^{*} \gamma_{K}\left(\mathbb{F}^{n}\right)$ and $(f \circ \pi)^{*} \gamma_{K}\left(\mathbb{F}^{n}\right)$ are topologically isomorphic. Thus, (b) implies (c).

We also have the following modification of Proposition 4.11 .

Proposition 4.13. Let $f: X \rightarrow \mathbb{G}_{K}\left(\mathbb{F}^{n}\right)$ be a continuous map whose restriction $\left.f\right|_{A}$ is stratified-regular. Assume the existence of a multiblowup $\pi: X^{\prime} \rightarrow X$ over $A$ such that the pullback $\mathbb{F}$-vector bundle $(f \circ \pi)^{*} \gamma_{K}\left(\mathbb{F}^{n}\right)$ on $X^{\prime}$ admits a stratified-algebraic structure. Then each neighborhood of $f$ in $\mathcal{C}\left(X, \mathbb{G}_{K}\left(\mathbb{F}^{n}\right)\right)$ contains a stratified-regular map $g: X \rightarrow \mathbb{G}_{K}\left(\mathbb{F}^{n}\right)$ with $\left.g\right|_{A}=\left.f\right|_{A}$. In particular, $f$ is homotopic to a stratified-regular map $h: X \rightarrow \mathbb{G}_{K}\left(\mathbb{F}^{n}\right)$ with $\left.h\right|_{A}=\left.f\right|_{A}$.

Proof. The map $\left.f\right|_{A}$ is $\mathcal{A}$-regular for some stratification $\mathcal{A}$ of $A$, and the $\mathbb{F}$-vector bundle $(f \circ \pi)^{*} \gamma_{K}\left(\mathbb{F}^{n}\right)$ admits an $\mathcal{S}$-algebraic structure for some stratification $\mathcal{S}$ of $X^{\prime}$. The collection $\mathcal{P}:=\left\{\pi^{-1}(T) \mid T \in \mathcal{A}\right\}$ is a stratification of $A^{\prime}:=\pi^{-1}(A)$, and the map $\left.(f \circ \pi)\right|_{A^{\prime}}$ is $\mathcal{P}$-regular. Moreover, the collection

$$
\mathcal{S}^{\prime}:=\{P \cap S \mid P \in \mathcal{P}, S \in \mathcal{S}\} \cup\left\{\left(X^{\prime} \backslash A^{\prime}\right) \cap S \mid S \in \mathcal{S}\right\}
$$

is a stratification of $X^{\prime}$, and the collection

$$
\mathcal{A}^{\prime}:=\left\{S^{\prime} \in \mathcal{S}^{\prime} \mid S^{\prime} \subseteq A^{\prime}\right\}
$$

is a stratification of $A^{\prime}$. By construction, $\mathcal{A}^{\prime}$ is a refinement of $\mathcal{P}$, and $\mathcal{S}^{\prime}$ is a refinement of $\mathcal{S}$. Consequently, the map $\left.(f \circ \pi)\right|_{A^{\prime}}$ is $\mathcal{A}^{\prime}$-regular, and the $\mathbb{F}$-vector bundle $(f \circ \pi)^{*} \gamma_{K}\left(\mathbb{F}^{n}\right)$ on $X^{\prime}$ admits an $\mathcal{S}^{\prime}$-algebraic structure. According to Corollary 4.6 (with $X=X^{\prime}, A=A^{\prime}$, 
$\left.\mathcal{X}=\mathcal{S}^{\prime}, \mathcal{A}=\mathcal{A}^{\prime}, f=f \circ \pi\right)$, there exists an $\mathcal{S}^{\prime}$-regular map $\varphi: X^{\prime} \rightarrow \mathbb{G}_{K}\left(\mathbb{F}^{n}\right)$ that is arbitrarily close to $f \circ \pi$ and satisfies $\left.\varphi\right|_{A^{\prime}}=\left.(f \circ \pi)\right|_{A^{\prime}}$. The restriction $\pi_{A}: X^{\prime} \backslash A^{\prime} \rightarrow X \backslash A$ of $\pi$ is a biregular isomorphism. Since the map $\pi$ is proper, the map $g: X \rightarrow \mathbb{G}_{K}\left(\mathbb{F}^{n}\right)$, defined by

$$
g(x)= \begin{cases}\varphi\left(\pi_{A}^{-1}(x)\right) & \text { for } x \text { in } X \backslash A \\ f(x) & \text { for } x \text { in } A,\end{cases}
$$

is continuous. By construction, $g$ is close to $f$ and $\left.g\right|_{A}=\left.f\right|_{A}$. Moreover,

$$
\mathcal{X}:=\mathcal{A} \cup\left\{\pi_{A}\left(S^{\prime}\right) \mid S^{\prime} \in \mathcal{S}^{\prime}, S^{\prime} \subseteq X^{\prime} \backslash A^{\prime}\right\}
$$

is a stratification of $X$, and the map $g$ is $\mathcal{X}$-regular. If $g$ is sufficiently close to $f$, then $g$ is homotopic to $f$, and hence the existence of $h$ follows.

\section{Topological versus stratified-algebraic vector bundles}

Throughout this section, $X$ denotes a compact real algebraic variety. By making use of the notion of filtration, introduced in Section 2, we demonstrate how the behavior of a vector bundle on $X$ can be deduced from the behavior of its restrictions to Zariski closed subvarieties of $X$. This is crucial for, in particular, the proofs of Theorems 1.7, 1.8 and 1.9, given in the next section.

Proposition 5.1. Let $\xi$ be a topological $\mathbb{F}$-vector bundle on $X$ and let

$$
\mathcal{F}=\left(X_{-1}, X_{0}, \ldots, X_{m}\right)
$$

be a filtration of $X$. Assume that for each $i=0, \ldots, m$, there exists a multiblowup $\pi_{i}: X_{i}^{\prime} \rightarrow X_{i}$ over $X_{i-1}$ such that the pullback $\mathbb{F}$-vector bundle $\pi_{i}^{*}\left(\left.\xi\right|_{X_{i}}\right)$ on $X_{i}^{\prime}$ admits an algebraic structure. Then $\xi$ admits an $\overline{\mathcal{F}}$-algebraic structure.

Proof. Since $X$ is compact, we may assume that $\xi$ is of the form $\xi=f^{*} \gamma_{K}\left(\mathbb{F}^{n}\right)$ for some multi-Grassmannian $\mathbb{G}_{K}\left(\mathbb{F}^{n}\right)$ and continuous map $f: X \rightarrow \mathbb{G}_{K}\left(\mathbb{F}^{n}\right)$. It suffices to prove that $f$ is homotopic to an $\overline{\mathcal{F}}$-regular map, cf. Corollary 4.7 .

Assertion. For each $i=0, \ldots, m$, there exists a continuous map $g_{i}: X \rightarrow \mathbb{G}_{K}\left(\mathbb{F}^{n}\right)$ homotopic to $f$ and such that the restriction of $g_{i}$ to $X_{j} \backslash X_{j-1}$ is a regular map for $0 \leq j \leq i$.

If the Assertion holds, then the map $g_{m}$ is $\overline{\mathcal{F}}$-regular and the proof is complete. We prove the Assertion by induction on $i$. Recall that $\left(X, X_{i}\right)$ is a polyhedral pair for $0 \leq i \leq m$, cf. [9, Corollary 9.3.7]. Since $X_{0}^{\prime}=X_{0}$ and $\pi_{0}: X_{0}^{\prime} \rightarrow X_{0}$ is the identity map, the $\mathbb{F}$-vector bundle $\left.\xi\right|_{X_{0}}$ on $X_{0}$ admits an algebraic structure. According to Proposition 4.11 (with $X=X_{0}, A=\varnothing$ ), the map $\left.f\right|_{X_{0}}$ is homotopic to a regular map $\varphi: X_{0} \rightarrow \mathbb{G}_{K}\left(\mathbb{F}^{n}\right)$. The homotopy extension theorem [43, p. 118, Corollary 5] implies the existence of a continuous map $g_{0}: X \rightarrow \mathbb{G}_{K}\left(\mathbb{F}^{n}\right)$ homotopic to $f$ and satisfying $\left.g_{0}\right|_{X_{0}}=\varphi$. We now suppose that $0 \leq i \leq m-1$ and the map $g_{i}$ satisfying the required conditions has already been constructed. In particular, the map $\left.g_{i}\right|_{X_{i}}$ is stratified-regular. Since the maps $\left.g_{i}\right|_{X_{i+1}}$ and $\left.f\right|_{X_{i+1}}$ are homotopic, the $\mathbb{F}$-vector bundles $\left(\left.g_{i}\right|_{X_{i+1}}\right)^{*} \gamma_{K}\left(\mathbb{F}^{n}\right)$ and $\left(\left.f\right|_{X_{i+1}}\right)^{*} \gamma_{K}\left(\mathbb{F}^{n}\right)=\left.\xi\right|_{X_{i+1}}$ are topologically isomorphic. Consequently, the $\mathbb{F}$-vector bundle

$$
\pi_{i+1}^{*}\left(\left(\left.g_{i}\right|_{X_{i+1}}\right)^{*} \gamma_{K}\left(\mathbb{F}^{n}\right)\right)=\left(\left(\left.g_{i}\right|_{X_{i+1}}\right) \circ \pi_{i+1}\right)^{*} \gamma_{K}\left(\mathbb{F}^{n}\right)
$$


on $X_{i+1}^{\prime}$ admits an algebraic structure, being topologically isomorphic to $\pi_{i+1}^{*}\left(\left.\xi\right|_{X_{i+1}}\right)$. According to Proposition 4.11 (with $X=X_{i+1}, A=X_{i}$ ), the map $\left.g_{i}\right|_{X_{i+1}}$ is homotopic to a continuous map $\psi: \bar{X}_{i+1} \rightarrow \mathbb{G}_{K}\left(\mathbb{F}^{n}\right)$ such that $\left.\psi\right|_{X_{i}}=\left.g_{i}\right|_{X_{i}}$ and the restriction of $\psi$ to $X_{i+1} \backslash X_{i}$ is a regular map. By the homotopy extension theorem, there exists a continuous map $g_{i+1}: X \rightarrow \mathbb{G}_{K}\left(\mathbb{F}^{n}\right)$ homotopic to $g_{i}$ (hence homotopic to $f$ ) and satisfying $\left.g_{i+1}\right|_{X_{i+1}}=\psi$. This completes the proof of the Assertion.

Theorem 5.2. Let $\xi$ be a topological $\mathbb{F}$-vector bundle on $X$ and let $\mathcal{F}=\left(X_{-1}, X_{0}, \ldots, X_{m}\right)$ be a filtration of $X$. If the stratification $\overline{\mathcal{F}}$ is nonsingular, then the following conditions are equivalent:

(a) The $\mathbb{F}$-vector bundle $\xi$ admits an $\overline{\mathcal{F}}$-algebraic structure.

(b) For each $i=0, \ldots, m$, there exists a multiblowup $\pi_{i}: X_{i}^{\prime} \rightarrow X_{i}$ over $X_{i-1}$ such that $X_{i}^{\prime}$ is a nonsingular variety and the pullback $\mathbb{F}$-vector bundle $\pi_{i}^{*}\left(\left.\xi\right|_{X_{i}}\right)$ on $X_{i}^{\prime}$ admits an algebraic structure.

Proof. Suppose that (a) holds. In view of Propositions 2.2 and 3.3 , we may assume that $\xi$ is of the form $\xi=f^{*} \gamma_{K}\left(\mathbb{F}^{n}\right)$ for some multi-Grassmannian $\mathbb{G}_{K}\left(\mathbb{F}^{n}\right)$ and $\overline{\mathcal{F}}$-regular map $f: X \rightarrow \mathbb{G}_{K}\left(\mathbb{F}^{n}\right)$. Set $f_{i}:=\left.f\right|_{X_{i}}$ for $0 \leq i \leq m$. The map $\left.f_{i}\right|_{X_{i-1}}$ is stratified-regular, and the restriction of $f_{i}$ to $X_{i} \backslash X_{i-1}$ is a regular map. The existence of $\pi_{i}$ as in (b) follows from Corollary 4.12 (with $X=X_{i}, A=X_{i-1}$ ). Thus, (a) implies (b). According to Proposition 5.1, (b) implies (a).

Theorem 5.2 implies the following characterization of topological $\mathbb{F}$-vector bundles on $X$ admitting a stratified-algebraic structure.

Corollary 5.3. For a topological $\mathbb{F}$-vector bundle $\xi$ on $X$, the following conditions are equivalent:

(a) The $\mathbb{F}$-vector bundle $\xi$ admits a stratified-algebraic structure.

(b) There exists a filtration $\mathcal{F}=\left(X_{-1}, X_{0}, \ldots, X_{m}\right)$ of $X$, with $\overline{\mathcal{F}}$ a nonsingular stratification, and for each $i=0, \ldots, m$, there exists a multiblowup $\pi_{i}: X_{i}^{\prime} \rightarrow X_{i}$ over $X_{i-1}$ such that $X_{i}^{\prime}$ is a nonsingular variety and the pullback $\mathbb{F}$-vector bundle $\pi_{i}^{*}\left(\left.\xi\right|_{X_{i}}\right)$ on $X_{i}^{\prime}$ admits an algebraic structure.

Proof. It suffices to combine Proposition 3.5 and Theorem 5.2 .

The following result will also prove to be useful.

Theorem 5.4. For a topological $\mathbb{F}$-vector bundle $\xi$ on $X$, the following conditions are equivalent:

(a) The $\mathbb{F}$-vector bundle $\xi$ admits a stratified-algebraic structure.

(b) There exists a filtration $\mathcal{F}=\left(X_{-1}, X_{0}, \ldots, X_{m}\right)$ of $X$, and for each $i=0, \ldots, m$, there exists a multiblowup $\pi_{i}: X_{i}^{\prime} \rightarrow X_{i}$ over $X_{i-1}$ such that the pullback $\mathbb{F}$-vector bundle $\pi_{i}^{*}\left(\left.\xi\right|_{X_{i}}\right)$ on $X_{i}^{\prime}$ admits a stratified-algebraic structure. 
Proof. By Corollary 5.3, (a) implies (b). It suffices to prove that (b) implies (a). The proof is similar to the proof of Proposition 5.1.

Suppose that (b) holds. Since $X$ is compact, we may assume that $\xi$ is of the form

$$
\xi=f^{*} \gamma_{K}\left(\mathbb{F}^{n}\right)
$$

for some multi-Grassmannian $\mathbb{G}_{K}\left(\mathbb{F}^{n}\right)$ and continuous map $f: X \rightarrow \mathbb{G}_{K}\left(\mathbb{F}^{n}\right)$. It remains to prove that $f$ is homotopic to a stratified-regular map, cf. Theorem 4.10 (with $A=\varnothing$ ).

Assertion. For each $i=0, \ldots, m$, there exists a continuous map $g_{i}: X \rightarrow \mathbb{G}_{K}\left(\mathbb{F}^{n}\right)$ homotopic to $f$ and such that the restriction $\left.g_{i}\right|_{X_{i}}$ is a stratified-regular map.

If the Assertion holds, then the map $g_{m}$ is stratified-regular and the proof is complete. We prove the Assertion by induction on $i$. Since $X_{0}^{\prime}=X_{0}$ and $\pi_{0}: X_{0}^{\prime} \rightarrow X_{0}$ is the identity map, the $\mathbb{F}$-vector bundle $\left.\xi\right|_{X_{0}}$ on $X_{0}$ admits a stratified-algebraic structure. According to Proposition 4.13 (with $X=X_{0}$ and $A=\varnothing$ ), the map $\left.f\right|_{X_{0}}$ is homotopic to a stratifiedregular map $\varphi: X_{0} \rightarrow \mathbb{G}_{K}\left(\mathbb{F}^{n}\right)$. The homotopy extension theorem implies the existence of a continuous map $g_{0}: X \rightarrow \mathbb{G}_{K}\left(\mathbb{F}^{n}\right)$ homotopic to $f$ and satisfying $\left.g_{0}\right|_{X_{0}}=\varphi$. We now suppose that $0 \leq i \leq m-1$ and the map $g_{i}$ satisfying the required conditions has already been constructed. In particular, the map $\left.g_{i}\right|_{X_{i}}$ is stratified-regular. Since the maps $\left.g_{i}\right|_{X_{i+1}}$ and $\left.f\right|_{X_{i+1}}$ are homotopic, the $\mathbb{F}$-vector bundles $\left(\left.g_{i}\right|_{X_{i+1}}\right)^{*} \gamma_{K}\left(\mathbb{F}^{n}\right)$ and $\left(\left.f\right|_{X_{i+1}}\right)^{*} \gamma_{K}\left(\mathbb{F}^{n}\right)=\left.\xi\right|_{X_{i+1}}$ are topologically isomorphic. Consequently, the $\mathbb{F}$-vector bundle

$$
\pi_{i+1}^{*}\left(\left(\left.g_{i}\right|_{X_{i+1}}\right)^{*} \gamma_{K}\left(\mathbb{F}^{n}\right)\right)=\left(\left(\left.g_{i}\right|_{X_{i+1}}\right) \circ \pi_{i+1}\right)^{*} \gamma_{K}\left(\mathbb{F}^{n}\right)
$$

on $X_{i+1}^{\prime}$ admits a stratified-algebraic structure, being topologically isomorphic to $\pi_{i+1}^{*}\left(\left.\xi\right|_{X_{i+1}}\right)$. According to Proposition 4.13 (with $X=X_{i+1}, A=X_{i}$ ), the map $\left.g_{i}\right|_{X_{i+1}}$ is homotopic to a stratified-regular map $\psi: X_{i+1} \rightarrow \mathbb{G}_{K}\left(\mathbb{F}^{n}\right)$ satisfying $\left.\psi\right|_{X_{i}}=\left.g_{i}\right|_{X_{i}}$. By the homotopy extension theorem, there exists a continuous map $g_{i+1}: X \rightarrow \mathbb{G}_{K}\left(\mathbb{F}^{n}\right)$ homotopic to $g_{i}$ (hence homotopic to $f$ ) and satisfying $\left.g_{i+1}\right|_{X_{i+1}}=\psi$. This completes the proof of the Assertion.

\section{Blowups and vector bundles}

We need certain constructions involving smooth (of class $\mathcal{C}^{\infty}$ ) manifolds. All smooth manifolds are assumed to be paracompact and without boundary. Submanifolds are supposed to be closed subsets of the ambient manifold. For any smooth manifold $M$, the total space of the tangent bundle to $M$ is denoted by $T M$. If $Z$ is a smooth submanifold of $M$, then $N_{Z} M$ denotes the total space of the normal bundle to $Z$ in $M$. Thus for each point $x$ in $Z$, the fiber $\left(N_{Z} M\right)_{x}$ is equal to $T M_{x} / T Z_{x}$.

Let $\theta$ be a smooth $\mathbb{R}$-vector bundle of rank $r$ on $M$, and let $s: M \rightarrow \theta$ be a smooth section transverse to the zero section. Then the zero locus $Z(s)$ of $s$ is a smooth submanifold of $M$, which is either empty or of codimension $r$. In order to have a convenient reference, we record the following well known fact.

Lemma 6.1. With notation as above, the restriction $\left.\theta\right|_{Z(s)}$ is smoothly isomorphic to the normal bundle to $Z(s)$ in $M$.

Proof. Let $E$ denote the total space of $\theta$, and let $Z:=Z(s)$. We regard $M$ as a smooth submanifold of $E$, identifying it with the image by the zero section. We identify $\theta$ with 
the normal bundle to $M$ in $E$. Since $Z=s^{-1}(M)$ and $s$ is transverse to $M$ in $E$, for each point $z$ in $Z$, the differential $d s_{z}: T M_{z} \rightarrow T E_{z}$ induces an $\mathbb{R}$-linear isomorphism

$$
\left(N_{Z} M\right)_{z} \rightarrow\left(N_{M} E\right)_{z}=E_{z}
$$

The proof is complete.

For any smooth submanifold $D$ of $M$ of codimension 1 , there exists a smooth $\mathbb{R}$-line bundle $\lambda(D)$ on $M$ with a smooth section $s_{D}: M \rightarrow \lambda(D)$ such that $Z\left(s_{D}\right)=D$ and $s_{D}$ is transverse to the zero section. Both $\lambda(D)$ and $s_{D}$ are constructed as in algebraic geometry, by regarding $D$ as a $\mathcal{C}^{\infty}$ divisor on $M$. Explicitly, if $\left\{U_{i}\right\}$ is an open cover of $M$ and $\left\{f_{i}: U_{i} \rightarrow \mathbb{R}\right\}$ is a collection of smooth local equations for $D$, then $\lambda(D)$ is determined by the transition functions $g_{i j}:=f_{i} / f_{j}: U_{i} \cap U_{j} \rightarrow \mathbb{R} \backslash\{0\}$, whereas $s_{D}$ is determined by the functions $f_{i}$. On the other hand, if $\lambda(D)$ and $s_{D}$ are already given, then $s_{D}$ gives rise to smooth local equations for $D$, and hence $\lambda(D)$ is uniquely determined up to smooth isomorphism. It is also convenient to set $\lambda(\varnothing):=\varepsilon_{M}^{1}(\mathbb{R})$.

For any smooth submanifold $Z$ of $M$, we denote by

$$
\pi(M, Z): B(M, Z) \rightarrow M
$$

the blowup of $M$ with center $Z$ (cf. 2] for basic properties of this construction). Recall that as a point set, $B(M, Z)$ is the union of $M \backslash Z$ and the total space $\mathbb{P}\left(N_{Z} M\right)$ of the projective bundle associated with the normal bundle to $Z$ in $M$. The map $\pi(M, Z)$ is the identity on $M \backslash Z$ and the bundle projection $\mathbb{P}\left(N_{Z} M\right) \rightarrow Z$ on $\mathbb{P}\left(N_{Z} M\right)$. On $B(M, Z)$ there is a natural smooth manifold structure, and $\pi(M, Z)$ is a smooth map. If $Z \neq \varnothing$ and $\operatorname{codim}_{M} Z \geq 1$, then $\pi(M, Z)^{-1}(Z)=\mathbb{P}\left(N_{Z} M\right)$ is a smooth submanifold of $B(M, Z)$ of codimension 1 .

Proposition 6.2. Let $M$ be a smooth manifold. Let $\theta$ be a smooth $\mathbb{R}$-vector bundle of positive rank on $M$, and let $s: M \rightarrow \theta$ be a smooth section transverse to the zero section. If $Z:=Z(s)$ and $\pi(M, Z): B(M, Z) \rightarrow M$ is the blowup of $M$ with center $Z$, then the pullback $\mathbb{R}$-vector bundle $\pi(M, Z)^{*} \theta$ on $B(M, Z)$ contains a smooth $\mathbb{R}$-line subbundle smoothly isomorphic to $\lambda(D)$, where $D:=\pi(M, Z)^{-1}(Z)$.

Proof. Let $E$ be the total space of $\xi$ and let $p: E \rightarrow M$ be the bundle projection. We regard $M$ as a smooth submanifold of $E$ and identify the normal bundle to $M$ in $E$ with $\theta$. Thus as a point set $B(E, M)$ is the union of $E \backslash M$ and $\mathbb{P}(E)$, while

$$
\pi(E, M): B(E, M) \rightarrow E
$$

is the identity on $E \backslash M$ and the bundle projection $\mathbb{P}(E) \rightarrow M$ on $\mathbb{P}(E)$. Here $\mathbb{P}(E)$ is the total space of the projective bundle on $M$ associated with $\theta$. The pullback smooth $\mathbb{R}$ vector bundle $(p \circ \pi(E, M))^{*} \theta$ on $B(E, M)$ contains a smooth $\mathbb{R}$-line subbundle $\lambda$ defined as follows. The fiber of $\lambda$ over a point $e$ in $E \backslash M$ is the line $\{e\} \times(\mathbb{R} e)$, and the restriction $\left.\lambda\right|_{\mathbb{P}(E)}$ is the tautological $\mathbb{R}$-line bundle on $\mathbb{P}(E)$.

Since $s$ is transverse to $M$ in $E$, for each point $z$ in $Z$, the differential

$$
d s_{z}: T M_{z} \rightarrow(T E)_{z}
$$

induces an $\mathbb{R}$-linear isomorphism

$$
\bar{d} s_{z}:\left(N_{Z} M\right)_{z} \rightarrow\left(N_{M} E\right)_{z}=E_{z}
$$


between the fibres over $z$ of the normal bundle to $Z$ in $M$ and the normal bundle to $M$ in $E$. Define $\bar{s}: B(M, Z) \rightarrow B(E, M)$ by $\bar{s}(x)=x$ for all $x$ in $M \backslash Z$ and $\bar{s}(l)=\bar{d} s_{z}(l)$ for all $l$ in $\mathbb{P}\left(N_{Z} M\right)_{z}$ (thus, $\bar{s}(l)$ is in the fiber $\left.\mathbb{P}(E)_{z}\right)$. By construction, $\bar{s}$ is a smooth map satisfying

$$
p \circ \pi(E, M) \circ \bar{s}=\pi(M, Z) .
$$

Hence $\bar{s}^{*} \lambda$ is a smooth $\mathbb{R}$-line subbundle of

$$
\bar{s}^{*}\left((p \circ \pi(E, M))^{*} \theta\right)=(p \circ \pi(E, M) \circ \bar{s})^{*} \theta=\pi(M, Z)^{*} \theta .
$$

It remains to prove that the $\mathbb{R}$-line bundles $\bar{s}^{*} \lambda$ and $\lambda(D)$ are smoothly isomorphic. To this end, it suffices to construct a smooth section $u: B(M, Z) \rightarrow \bar{s}^{*} \lambda$ that is transverse to the zero section and satisfies $Z(u)=D$. Such a section can be obtained as follows. The smooth section $v: B(E, M) \rightarrow \lambda$, defined by $v(e)=(e, e)$ for all $e$ in $E \backslash M$ and $\left.v\right|_{\mathbb{P}(E)}=0$, is transverse to the zero section and satisfies $Z(v)=\mathbb{P}(E)$. On the other hand, the smooth map $\bar{s}: B(M, Z) \rightarrow B(E, M)$ is transverse to $\mathbb{P}(E)$ in $B(E, M)$ and $\bar{s}^{-1}(\mathbb{P}(E))=\pi(M, Z)^{-1}(Z)=D$. Consequently, the smooth section

$$
u:=\bar{s}^{*} v: B(M, Z) \rightarrow \bar{s}^{*} \lambda
$$

satisfies the required conditions.

Let $X$ be a nonsingular real algebraic variety. For any nonsingular Zariski closed subvariety $D$ of $X$ of codimension 1 , there exists an algebraic $\mathbb{R}$-line bundle $\lambda(D)$ on $X$ with an algebraic section $s_{D}: X \rightarrow \lambda(D)$ such that $Z\left(s_{D}\right)=D$ and $s_{D}$ is transverse to the zero section. The $\mathbb{R}$-line bundle $\lambda(D)$ is uniquely determined up to algebraic isomorphism.

Recall that any algebraic $\mathbb{R}$-vector bundle $\theta$ on $X$ has an algebraic section transverse to the zero section. Indeed, $\theta$ is generated by finitely many global algebraic sections $s_{1}, \ldots, s_{n}$, cf. [9, Theorem 12.1.7] or Proposition 3.7. According to the transversality theorem, for a general point $\left(t_{1}, \ldots, t_{n}\right)$ in $\mathbb{R}^{n}$, the algebraic section $t_{1} s_{1}+\cdots+t_{n} s_{n}$ is transverse to the zero section.

If $Z$ is a nonsingular Zariski closed subvariety of $X$, the $\mathcal{C}^{\infty}$ blowup

$$
\pi(X, Z): B(X, Z) \rightarrow X
$$

can be identified with the algebraic blowup, cf. [2, Lemma 2.5.5].

The following consequence of Proposition 6.2 will play an important role.

Corollary 6.3. Let $X$ be a nonsingular real algebraic variety. Let $\theta$ be a smooth $\mathbb{R}$-vector bundle of positive rank on $X$, and let $s: X \rightarrow \theta$ be a smooth section transverse to the zero section. Assume that $Z:=Z(s)$ is a nonsingular Zariski closed subvariety of $X$. If $\pi: X^{\prime} \rightarrow X$ is the blowup with center $Z$, then the smooth $\mathbb{R}$-vector bundle $\pi^{*} \theta$ on $X^{\prime}$ contains a smooth $\mathbb{R}$-line subbundle $\lambda$ which is smoothly isomorphic to an algebraic $\mathbb{R}$-line bundle on $X^{\prime}$. In particular, $\lambda$ admits an algebraic structure.

Proof. Let $D:=\pi^{-1}(Z)$. Either $D=\varnothing$ or $D$ is a nonsingular Zariski closed subvariety of $X^{\prime}$ of codimension 1 . Consequently, $\lambda(D)$ is an algebraic $\mathbb{R}$-line bundle on $X^{\prime}$. The proof is complete in view of Proposition 6.2.

There is also an algebraic-geometric counterpart of Proposition 6.2. 
Proposition 6.4. Let $X$ be a nonsingular real algebraic variety. Let $\theta$ be an algebraic $\mathbb{R}$-vector bundle of positive rank on $X$, and let $s: X \rightarrow \theta$ be an algebraic section transverse to the zero section. If $\pi: X^{\prime} \rightarrow X$ is the blowup with center $Z:=Z(s)$, then the pullback $\mathbb{R}$-vector bundle $\pi^{*} \theta$ on $X^{\prime}$ contains an algebraic $\mathbb{R}$-line subbundle which is algebraically isomorphic to $\lambda(D)$, where $D:=\pi^{-1}(Z)$.

Proof. The proof of Proposition 6.2 can be carried over to the algebraic-geometric setting.

For any $\mathbb{R}$-vector bundle $\theta$, we denote by $\mathbb{C} \otimes \theta$ and $\mathbb{H} \otimes \theta$ the complexification and the quaternionification of $\theta$. In order to have the uniform notation, we also set $\mathbb{R} \otimes \theta:=\theta$. Thus, $\mathbb{F} \otimes \theta$ is an $\mathbb{F}$-vector bundle.

The following lemma leads directly to the proof of Theorem 1.7

Lemma 6.5. Let $X$ be a compact nonsingular real algebraic variety. If $\xi$ is a topological $\mathbb{F}$-vector bundle on $X$ such that the $\mathbb{R}$-vector bundle $\xi_{\mathbb{R}}$ admits an algebraic structure, then $\xi$ admits a stratified-algebraic structure as an $\mathbb{F}$-vector bundle.

Proof. We may assume without loss of generality that the variety $X$ is irreducible. Then $\xi$ is of constant rank since $\xi_{\mathbb{R}}$ admits an algebraic structure. We use induction on $r:=\operatorname{rank} \xi$. Obviously, the assertion holds if $r=0$. Suppose that $r \geq 1$ and the assertion holds for all topological $\mathbb{F}$-vector bundles of rank at most $r-1$, defined on compact nonsingular real algebraic varieties. Let $\theta$ be an algebraic $\mathbb{R}$-vector bundle on $X$ that is topologically isomorphic to $\xi_{\mathbb{R}}$. Let $s: X \rightarrow \theta$ be an algebraic section transverse to the zero section and let $Z:=Z(s)$. Then either $Z=\varnothing$ or $Z$ is a nonsingular Zariski closed subvariety of $X$ of codimension $d(\mathbb{F}) r$. Let $\pi: X^{\prime} \rightarrow X$ be the blowup of $X$ with center $Z$. According to Proposition 6.4 the pullback $\mathbb{R}$-vector bundle $\pi^{*} \theta$ on $X^{\prime}$ contains an algebraic $\mathbb{R}$-line subbundle $\lambda$. Hence, $\pi^{*}\left(\xi_{\mathbb{R}}\right)=\left(\pi^{*} \xi\right)_{\mathbb{R}}$ contains a topological $\mathbb{R}$-line subbundle $\mu$ that is topologically isomorphic to $\lambda$. Since $\pi^{*} \xi$ is an $\mathbb{F}$-vector bundle, it contains a topological $\mathbb{F}$-line subbundle $\lambda^{\prime}$ isomorphic to $\mathbb{F} \otimes \mu$. By construction, $\lambda^{\prime}$ is topologically isomorphic to the algebraic $\mathbb{F}$-line bundle $\mathbb{F} \otimes \lambda$. In particular, the $\mathbb{F}$-line bundle $\lambda^{\prime}$ admits an algebraic structure, and the $\mathbb{F}$-vector bundle $\pi^{*} \xi$ can be expressed as

$$
\pi^{*} \xi=\lambda^{\prime} \oplus \xi^{\prime}
$$

where $\xi^{\prime}$ is a topological $\mathbb{F}$-vector bundle on $X^{\prime}$ of rank $r-1$. Note that the $\mathbb{R}$-vector bundle

$$
\pi^{*}\left(\xi_{\mathbb{R}}\right)=\left(\pi^{*} \xi\right)_{\mathbb{R}}=\lambda_{\mathbb{R}}^{\prime} \oplus \xi_{\mathbb{R}}^{\prime}
$$

admits an algebraic structure. Since $\lambda_{\mathbb{R}}^{\prime}$ admits an algebraic structure, there exists an algebraic $\mathbb{R}$-vector bundle $\eta$ on $X^{\prime}$ such that the direct sum $\eta \oplus \lambda_{\mathbb{R}}^{\prime}$ is topologically isomorphic to a trivial algebraic $\mathbb{R}$-vector bundle $\varepsilon$ on $X^{\prime}$, cf. [9, Theorem 12.1.7] or Proposition 3.7. Consequently, the $\mathbb{R}$-vector bundle $\varepsilon \oplus \xi_{\mathbb{R}}^{\prime}$ admits an algebraic structure, being topologically isomorphic to $\eta \oplus \pi^{*}\left(\xi_{\mathbb{R}}\right)$. According to [9, Proposition 12.3.5] or Corollary 3.13, the $\mathbb{R}$-vector bundle $\xi_{\mathbb{R}}^{\prime}$ admits an algebraic structure. Now, by the induction hypothesis, the $\mathbb{F}$-vector bundle $\xi^{\prime}$ on $X^{\prime}$ admits a stratified-algebraic structure. Consequently, the $\mathbb{F}$-vector bundle $\pi^{*} \xi$ on $X^{\prime}$ admits a stratified-algebraic structure.

Consider the topological $\mathbb{F}$-vector bundle $\left.\xi\right|_{Z}$ on $Z$. Since the $\mathbb{R}$-vector bundle

$$
\left(\left.\xi\right|_{Z}\right)_{\mathbb{R}}=\left.\left(\xi_{\mathbb{R}}\right)\right|_{Z}
$$

is topologically isomorphic to $\left.\theta\right|_{Z}$, the construction above can be repeated with $\left.\xi\right|_{Z}$ substituted for $\xi$. By continuing this process, we obtain a filtration $\mathcal{F}=\left(X_{-1}, X_{0}, \ldots, X_{m}\right)$ of $X$ such that for each $i=0, \ldots, m$, the following two conditions are satisfied: 
(*) $X_{i}$ is a nonsingular Zariski closed subvariety of $X$;

(**) If $\pi_{i}: X_{i}^{\prime} \rightarrow X_{i}$ is the blowup of $X_{i}$ with center $X_{i-1}$, then the pullback $\mathbb{F}$-vector bundle $\pi_{i}^{*}\left(\left.\xi\right|_{X_{i}}\right)$ on $X_{i}^{\prime}$ admits a stratified-algebraic structure.

According to Theorem 5.4 , the topological $\mathbb{F}$-vector bundle $\xi$ admits a stratified-algebraic structure, as required.

Proof of Theorem 1.7. Let $\xi$ be a topological $\mathbb{F}$-vector bundle on $X$. Obviously, if $\xi$ admits a stratified-algebraic structure, then so does $\xi_{\mathbb{R}}$.

Suppose now that $\xi_{\mathbb{R}}$ admits a stratified-algebraic structure. We prove by induction on $\operatorname{dim} X$ that $\xi$ admits a stratified-algebraic structure. In view of Theorem 1.6, there exists a birational multiblowup $\pi: X^{\prime} \rightarrow X$ such that $X^{\prime}$ is a nonsingular variety and the $\mathbb{R}$-vector bundle $\pi^{*}\left(\xi_{\mathbb{R}}\right)=\left(\pi^{*} \xi\right)_{\mathbb{R}}$ on $X^{\prime}$ admits an algebraic structure. By Lemma 6.5. the $\mathbb{F}$-vector bundle $\pi^{*} \xi$ on $X^{\prime}$ admits a stratified-algebraic structure. Let $A$ be a Zariski closed subvariety such that $\operatorname{dim} A<\operatorname{dim} X$ and $\pi$ is a multiblowup over $A$. The $\mathbb{R}$ vector bundle $\left(\left.\xi\right|_{A}\right)_{\mathbb{R}}=\left.\left(\xi_{\mathbb{R}}\right)\right|_{A}$ on $A$ admits a stratified-algebraic structure. Hence, by the induction hypothesis, the $\mathbb{F}$-vector bundle $\left.\xi\right|_{A}$ on $A$ admits a stratified-algebraic structure. Note that $\mathcal{F}=(\varnothing, A, X)$ is a filtration of $X$. According to Theorem 5.4 , the $\mathbb{F}$-vector bundle $\xi$ admits a stratified-algebraic structure.

We show next that Theorem 1.7 can be restated as an approximation result. If $K$ is a nonempty finite collection of nonnegative integers, we set $d(\mathbb{F}) K:=\{d(\mathbb{F}) k \mid k \in K\}$. Any $\mathbb{F}$-vector subspace $V$ of $\mathbb{F}^{n}$ can be regarded as an $\mathbb{R}$-vector subspace of $\mathbb{R}^{d(\mathbb{F}) n}$, which is indicated by $V_{\mathbb{R}}$. The correspondence $V \rightarrow V_{\mathbb{R}}$ gives rise to a regular map

$$
i: \mathbb{G}_{K}\left(\mathbb{F}^{n}\right) \rightarrow \mathbb{G}_{d(\mathbb{F}) K}\left(\mathbb{R}^{d(\mathbb{F}) n}\right) .
$$

Theorem 6.6. Let $X$ be a compact real algebraic variety. For a continuous map

$$
f: X \rightarrow \mathbb{G}_{K}\left(\mathbb{F}^{n}\right),
$$

the following conditions are equivalent:

(a) The map $f: X \rightarrow \mathbb{G}_{K}\left(\mathbb{F}^{n}\right)$ can be approximated by stratified-regular maps.

(b) The map $i \circ f: X \rightarrow \mathbb{G}_{d(\mathbb{F}) K}\left(\mathbb{R}^{d(\mathbb{F}) n}\right)$ can be approximated by stratified-regular maps.

Proof. Condition (a) implies (b), the map $i$ being regular. Since

$$
\left(\gamma_{K}\left(\mathbb{F}^{n}\right)\right)_{\mathbb{R}}=i^{*}\left(\gamma_{d(\mathbb{F}) K}\left(\mathbb{R}^{d(\mathbb{F}) n}\right)\right),
$$

we get

$$
\left(f^{*} \gamma_{K}\left(\mathbb{F}^{n}\right)\right)_{\mathbb{R}}=f^{*}\left(\left(\gamma_{K}\left(\mathbb{F}^{n}\right)\right)_{\mathbb{R}}\right)=(i \circ f)^{*} \gamma_{d(\mathbb{F}) K}\left(\mathbb{R}^{d(\mathbb{F}) n}\right) .
$$

Hence, according to Theorem 4.10 (with $\mathbb{F}=\mathbb{R}, f=i \circ f, A=\varnothing$ ), condition (b) implies that the $\mathbb{R}$-vector bundle $\left(f^{*} \gamma_{K}\left(\mathbb{F}^{n}\right)\right)_{\mathbb{R}}$ admits a stratified-algebraic structure. In view of Theorem 1.7, the $\mathbb{F}$-vector bundle $f^{*} \gamma_{K}\left(\mathbb{F}^{n}\right)$ admits a stratified-algebraic structure. Making again use of Theorem 4.10 (with $A=\varnothing$ ), we conclude that (b) implies (a).

In view of Theorem 4.10, one readily sees that Theorem 6.6 implies Theorem 1.7 . Proofs of Theorems 1.8 and 1.9 require further preparation. 
Lemma 6.7. Let $X$ be a compact nonsingular real algebraic variety and let $\xi$ be an adapted smooth $\mathbb{F}$-vector bundle on $X$. Then there exists a smooth section $s: X \rightarrow \xi$ transverse to the zero section and such that $Z(s)$ is a nonsingular Zariski locally closed subvariety of $X$.

Proof. According to the definition of an adapted vector bundle, we can choose a smooth section $u: X \rightarrow \xi$ transverse to the zero section and such that its zero locus $Z(u)$ is smoothly isotopic to a nonsingular Zariski locally closed subvariety $Z$ of $X$. Since any isotopy can be extended to a diffeotopy [28, p. 180, Theorem 1.3], there exists a smooth diffeomorphism $h: X \rightarrow X$ that is homotopic to the identity map $\mathbb{1}_{X}$ of $X$ and satisfies $h(Z)=Z(u)$. The pullback section $h^{*} u: X \rightarrow h^{*} \xi$ is transverse to the zero section and $Z\left(h^{*} u\right)=Z$. The proof is complete since the $\mathbb{F}$-vector bundle $h^{*} \xi$ is smoothly isomorphic to $\xi$, the maps $h$ and $\mathbb{1}_{X}$ being homotopic.

Lemma 6.8. Let $X$ be a nonsingular real algebraic variety. Let $\xi$ be a smooth $\mathbb{F}$-vector bundle on $X$, and let $s: X \rightarrow \xi$ be a smooth section transverse to the zero section and such that $Z:=Z(s)$ is a nonsingular Zariski locally closed subvariety of $X$. Let $V$ be the Zariski closure of $Z$ in $X$ and let $W:=V \backslash Z$. Then there exists a multiblowup $\rho: \tilde{X} \rightarrow X$ over $W$ such that $\tilde{Z}:=\rho^{-1}(Z)$ is a nonsingular Zariski closed subvariety of $\tilde{X}$, the pullback section $\rho^{*} s: \tilde{X} \rightarrow \rho^{*} \xi$ is transverse to the zero section, and $Z\left(\rho^{*} s\right)=\tilde{Z}$. Furthermore, if the variety $X$ is compact and the $\mathbb{F}$-vector bundles $\left.\xi\right|_{W}$ on $W$ and $\rho^{*} \xi$ on $\tilde{X}$ admit stratified-algebraic structures, then $\xi$ admits a stratified-algebraic structure.

Proof. Since $Z$ is closed in the Euclidean topology, it readily follows that $W$ is the singular locus of $V$. According to Hironaka's theorem on resolution of singularities [26, 31, there exists a multiblowup $\rho: \tilde{X} \rightarrow X$ over $W$ such that the Zariski closure $\tilde{Z}$ of $\rho^{-1}(Z)$ in $\tilde{X}$ is a nonsingular subvariety. In the case under consideration, $\tilde{Z}=\rho^{-1}(Z)$, the set $Z$ being closed in the Euclidean topology. Since the restriction $\rho_{W}: \tilde{X} \backslash \rho^{-1}(W) \rightarrow X \backslash W$ of $\rho$ is a biregular isomorphism, the pullback section $\rho^{*} s: \tilde{X} \rightarrow \rho^{*} \xi$ is transverse to the zero section. By construction, $Z\left(\rho^{*} s\right)=\tilde{Z}$. Note that $\mathcal{F}=(\varnothing, W, X)$ is a filtration of $X$. The last assertion in Lemma 6.8 follows from Theorem 5.4 .

Proof of Theorem 1.8. Let $\xi$ be an adapted smooth $\mathbb{F}$-line bundle on $X$. According to Lemma 6.7, there exists a smooth section $s: X \rightarrow \xi$ transverse to the zero section and such that $Z:=Z(s)$ is a nonsingular Zariski locally closed subvariety of $X$. Let $V$ be the Zariski closure of $Z$ in $X$ and let $W:=V \backslash Z$. Since $\xi$ is of rank 1 and $Z\left(\left.s\right|_{W}\right)=\varnothing$, it follows that the $\mathbb{F}$-vector bundle $\left.\xi\right|_{W}$ on $W$ is topologically trivial. In particular, $\left.\xi\right|_{W}$ admits a stratified-algebraic structure.

In view of Lemma 6.8, we may assume without loss of generality that $Z$ is a nonsingular and Zariski closed subvariety of $X$ (that is, $W=\varnothing$ ). Let $\pi: X^{\prime} \rightarrow X$ be the blowup with center $Z$. According to Corollary 6.3 , the smooth $\mathbb{R}$-vector bundle $\pi^{*}\left(\xi_{\mathbb{R}}\right)=\left(\pi^{*} \xi\right)_{\mathbb{R}}$ on $X^{\prime}$ contains a smooth $\mathbb{R}$-line subbundle $\lambda$ admitting an algebraic structure. Since $\pi^{*} \xi$ is an $\mathbb{F}$-line bundle, it follows that it is topologically isomorphic to $\mathbb{F} \otimes \lambda$. Consequently, $\pi^{*} \xi$ admits an algebraic structure. The restriction $\left.\left(\xi_{\mathbb{R}}\right)\right|_{Z}$ is smoothly isomorphic to the normal bundle to $Z$ in $X$, cf. Lemma 6.1. In particular, the $\mathbb{R}$-vector bundle $\left(\left.\xi\right|_{Z}\right)_{\mathbb{R}}=\left.\left(\xi_{\mathbb{R}}\right)\right|_{Z}$ admits an algebraic structure. By Theorem 1.7 , the $\mathbb{F}$-line bundle $\left.\xi\right|_{Z}$ admits a stratifiedalgebraic structure. Since $\mathcal{F}=(\varnothing, Z, X)$ is a filtration of $X$, Theorem 5.4 implies that $\xi$ admits a stratified-algebraic structure.

Proof of Theorem 1.9. Assume that both $\mathbb{F}$-vectors bundles $\xi$ and $\operatorname{det} \xi$ are adapted. According to Theorem 1.8 , the $\mathbb{F}$-line bundle $\operatorname{det} \xi$ admits a stratified algebraic structure. 
By Lemma 6.7, there exists a smooth section $s: X \rightarrow \xi$ transverse to the zero section and such that $Z:=Z(s)$ is a nonsingular Zariski locally closed subvariety of $X$. Let $V$ be the Zariski closure of $Z$ in $X$ and let $W:=V \backslash Z$. Since $\xi$ is of rank 2 and $Z\left(\left.s\right|_{W}\right)=\varnothing$, it follows that $\left.\xi\right|_{W}$ is topologically isomorphic to $\varepsilon_{W}^{1}(\mathbb{F}) \oplus \mu$ for some topological $\mathbb{F}$-line bundle $\mu$ on $W$. We have

$$
\left.(\operatorname{det} \xi)\right|_{W}=\operatorname{det}\left(\left.\xi\right|_{W}\right) \cong \operatorname{det}\left(\varepsilon_{W}^{1}(\mathbb{F}) \oplus \mu\right) \cong \varepsilon_{W}^{1}(\mathbb{F}) \otimes \mu \cong \mu .
$$

Consequently, the $\mathbb{F}$-vector bundle $\left.\xi\right|_{W}$ on $W$ admits a stratified-algebraic structure.

In view of Lemma 6.8, we may assume without loss of generality that $Z$ is a nonsingular and Zariski closed subvariety of $X$ (that is, $W=\varnothing$ ). Let $\pi: X^{\prime} \rightarrow X$ be the blowup with center $Z$. According to Corollary 6.3, the smooth $\mathbb{R}$-vector bundle $\pi^{*}\left(\xi_{\mathbb{R}}\right)=\left(\pi^{*} \xi\right)_{\mathbb{R}}$ contains a smooth $\mathbb{R}$-line subbundle $\lambda$ admitting an algebraic structure. Since $\pi^{*} \xi$ is an $\mathbb{F}$-vector bundle, it contains a smooth $\mathbb{F}$-line subbundle $\xi_{1}$ isomorphic to $\mathbb{F} \otimes \lambda$. Hence

$$
\pi^{*} \xi=\xi_{1} \oplus \xi_{2}
$$

where $\xi_{1}$ and $\xi_{2}$ are smooth $\mathbb{F}$-line bundles, and $\xi_{1}$ admits an algebraic structure. We have

$$
\pi^{*}(\operatorname{det} \xi)=\operatorname{det}\left(\pi^{*} \xi\right)=\operatorname{det}\left(\xi_{1} \oplus \xi_{2}\right) \cong \xi_{1} \otimes \xi_{2},
$$

which implies

$$
\xi_{1}^{\vee} \otimes \pi^{*}(\operatorname{det} \xi) \cong \xi_{1}^{\vee} \otimes \xi_{1} \otimes \xi_{2} \cong \xi_{2} .
$$

Thus, according to Propositions 2.6 and 3.15, $\xi_{2}$ admits a stratified-algebraic structure. Consequently, $\pi^{*} \xi$ admits a stratified-algebraic structure. The restriction $\left.\left(\xi_{\mathbb{R}}\right)\right|_{Z}$ is smoothly isomorphic to the normal bundle to $Z$ in $X$, cf. Lemma 6.1. In particular, the $\mathbb{R}$-vector bundle $\left(\left.\xi\right|_{Z}\right)_{\mathbb{R}}=\left.\left(\xi_{\mathbb{R}}\right)\right|_{Z}$ admits an algebraic structure. By Theorem 1.7 , the $\mathbb{F}$-vector bundle $\left.\xi\right|_{Z}$ admits a stratified-algebraic structure. Since $\mathcal{F}=(\varnothing, Z, X)$ is a filtration of $X$, Theorem 5.4 implies that $\xi$ admits a stratified-algebraic structure.

There are natural generalizations of Theorems 1.8 and 1.9 to vector bundles on an arbitrary compact real algebraic variety (not necessarily nonsingular).

First we recall a topological fact. If $M$ is a smooth manifold, then each topological $\mathbb{F}$-vector bundle $\eta$ on $M$ is topologically isomorphic to a smooth $\mathbb{F}$-vector bundle $\eta^{\infty}$, which is uniquely determined up to smooth isomorphism, cf. [28, p. 101, Theorem 3.5].

A topological $\mathbb{F}$-vector bundle $\xi$ on a compact nonsingular real algebraic variety $Y$ is said to be adapted if the smooth $\mathbb{F}$-vector bundle $\xi^{\infty}$ is adapted. In particular, $\xi$ is adapted if it admits an algebraic structure.

Theorem 6.9. Let $X$ be a compact real algebraic variety and let $\xi$ be a topological $\mathbb{F}$-line bundle on $X$. Then the following conditions are equivalent:

(a) The $\mathbb{F}$-line bundle $\xi$ admits a stratified-algebraic structure.

(b) There exists a filtration $\mathcal{F}=\left(X_{-1}, X_{0}, \ldots, X_{m}\right)$ of $X$ with $\overline{\mathcal{F}}$ a nonsingular stratification, and for each $i=0, \ldots, m$, there exists a multiblowup $\pi_{i}: X_{i}^{\prime} \rightarrow X_{i}$ over $X_{i-1}$ such that $X_{i}^{\prime}$ is a nonsingular variety and the pullback $\mathbb{F}$-line bundle $\pi_{i}^{*}\left(\left.\xi\right|_{X_{i}}\right)$ on $X_{i}^{\prime}$ is adapted.

Proof. According to Corollary 5.3, (a) implies (b). In view of Theorems 5.4 and 1.8 , (b) implies (a). 
Theorem 6.10. Let $X$ be a compact real algebraic variety and let $\xi$ be a topological $\mathbb{F}$ vector bundle of rank 2 on $X$, where $\mathbb{F}=\mathbb{R}$ of $\mathbb{F}=\mathbb{C}$. Then the following conditions are equivalent:

(a) The $\mathbb{F}$-vector bundle $\xi$ admits a stratified-algebraic structure.

(b) There exists a filtration $\mathcal{F}=\left(X_{-1}, X_{0}, \ldots, X_{m}\right)$ of $X$ with $\overline{\mathcal{F}}$ a nonsingular stratification, and for each $i=0, \ldots, m$, there exists a multiblowup $\pi_{i}: X_{i}^{\prime} \rightarrow X_{i}$ over $X_{i-1}$ such that $X_{i}^{\prime}$ is a nonsingular variety and the pullback $\mathbb{F}$-vector bundles $\pi_{i}^{*}\left(\left.\xi\right|_{X_{i}}\right)$ and $\pi_{i}^{*}\left(\left.(\operatorname{det} \xi)\right|_{X_{i}}\right)$ on $X_{i}^{\prime}$ are adapted.

Proof. According to Corollary 5.3, (a) implies (b). In view of Theorems 5.4 and 1.9 , (b) implies (a).

\section{Stratified-algebraic cohomology classes}

To begin with, we summarize basic properties of algebraic cohomology classes. Let $V$ be a compact nonsingular real algebraic variety. A cohomology class $v$ in $H^{k}(V ; \mathbb{Z} / 2)$ is said to be algebraic if the homology class Poincaré dual to it can be represented by a Zariski closed subvariety of $V$ of codimension $k$. The set $H_{\mathrm{alg}}^{k}(V ; \mathbb{Z} / 2)$ of all algebraic cohomology classes in $H^{k}(V ; \mathbb{Z} / 2)$ forms a subgroup. The direct sum $H_{\text {alg }}^{*}(V ; \mathbb{Z} / 2)$ of the groups $H_{\text {alg }}^{k}(V ; \mathbb{Z} / 2)$, for $k \geq 0$, is a subring of the cohomology $\operatorname{ring} H^{*}(V ; \mathbb{Z} / 2)$. For any algebraic $\mathbb{R}$-vector bundle $\xi$ on $V$, its $k$ th Stiefel-Whitney class $w_{k}(\xi)$ belongs to $H_{\text {alg }}^{k}(V ; \mathbb{Z} / 2)$ for every $k \geq 0$. If $h: V \rightarrow W$ is a regular map between compact nonsingular real algebraic varieties, then

$$
h^{*}\left(H_{\text {alg }}^{*}(W ; \mathbb{Z} / 2)\right) \subseteq H_{\text {alg }}^{*}(V ; \mathbb{Z} / 2) .
$$

For proofs of the facts listed above we refer to [20] or [1, 6, 9, 17].

We now introduce the main notion of this section. Let $X$ be a real algebraic variety. A cohomology class $u$ in $H^{k}(X ; \mathbb{Z} / 2)$ is said to be stratified-algebraic if there exists a stratified-regular map $\varphi: X \rightarrow V$, into a compact nonsingular real algebraic variety $V$, such that $u=\varphi^{*}(v)$ for some cohomology class $v$ in $H_{\text {alg }}^{k}(V ; \mathbb{Z} / 2)$.

Proposition 7.1. For any real algebraic variety $X$, the set $H_{\mathrm{str}}^{k}(X ; \mathbb{Z} / 2)$ of all stratifiedalgebraic cohomology classes in $H^{k}(X ; \mathbb{Z} / 2)$ forms a subgroup. Furthermore, the direct sum

$$
H_{\mathrm{str}}^{*}(X ; \mathbb{Z} / 2)=\bigoplus_{k \geq 0} H_{\mathrm{str}}^{k}(X ; \mathbb{Z} / 2)
$$

is a subring of the cohomology ring $H^{*}(X ; \mathbb{Z} / 2)$.

Proof. Let $\varphi_{i}: X \rightarrow V_{i}$ be a stratified-regular map into a compact nonsingular real algebraic variety $V_{i}$ for $i=1,2$. The stratified-regular map $\left(\varphi_{1}, \varphi_{2}\right): X \rightarrow V_{1} \times V_{2}$ satisfies $p_{i} \circ\left(\varphi_{1}, \varphi_{2}\right)=\varphi_{i}$, where $p_{i}: V_{1} \times V_{2} \rightarrow V_{i}$ is the canonical projection. If $v_{i}$ (resp. $\left.w_{i}\right)$ is a cohomology class in $H^{k}\left(V_{i} ; \mathbb{Z} / 2\right)\left(\right.$ resp. $\left.H^{k_{i}}\left(V_{i} ; \mathbb{Z} / 2\right)\right)$ for $i=1,2$, then

$$
\begin{aligned}
\varphi_{1}^{*}\left(v_{1}\right)+\varphi_{2}^{*}\left(v_{2}\right) & =\left(\varphi_{1}, \varphi_{2}\right)^{*}\left(p_{1}^{*}\left(v_{1}\right)+p_{2}^{*}\left(v_{2}\right)\right), \\
\varphi_{1}^{*}\left(w_{1}\right) \smile \varphi_{2}^{*}\left(w_{2}\right) & =\left(\varphi_{1}, \varphi_{2}\right)^{*}\left(p_{1}^{*}\left(w_{1}\right) \smile p_{2}^{*}\left(w_{2}\right)\right),
\end{aligned}
$$

where $\smile$ stands for the cup product. If $v_{i}$ and $w_{i}$ are algebraic cohomology classes, then the cohomology classes $p_{1}^{*}\left(v_{1}\right)+p_{2}^{*}\left(v_{2}\right)$ and $p_{1}^{*}\left(w_{1}\right) \smile p_{2}^{*}\left(w_{2}\right)$ are algebraic too. The proof is complete. 
Proposition 7.2. If $f: X \rightarrow Y$ is a stratified-regular map between real algebraic varieties, then

$$
f^{*}\left(H_{\mathrm{str}}^{*}(Y ; \mathbb{Z} / 2)\right) \subseteq H_{\mathrm{str}}^{*}(X ; \mathbb{Z} / 2) .
$$

Proof. Let $\psi: Y \rightarrow W$ be a stratified-regular map into a compact nonsingular real algebraic variety $W$. For any cohomology class $w$ in $H^{k}(W ; \mathbb{Z} / 2)$,

$$
f^{*}\left(\psi^{*}(w)\right)=(\psi \circ f)^{*}(w) .
$$

The proof is complete since $\psi \circ f$ is a stratified-regular map.

Proposition 7.3. Let $\xi$ be a stratified-algebraic $\mathbb{R}$-vector bundle on a real algebraic variety $X$. Then the $k$ th Stiefel-Whitney class $w_{k}(\xi)$ of $\xi$ belongs to $H_{\mathrm{str}}^{k}(X ; \mathbb{Z} / 2)$ for every $k \geq 0$.

Proof. According to Proposition 3.4, we may assume that $\xi$ is of the form $\xi=f^{*} \gamma_{K}\left(\mathbb{R}^{n}\right)$ for some multi-Grassmannian $\mathbb{G}_{K}\left(\mathbb{R}^{n}\right)$ and stratified-regular map $f: X \rightarrow \mathbb{G}_{K}\left(\mathbb{R}^{n}\right)$. Then $w_{k}(\xi)=f^{*}\left(w_{k}\left(\gamma_{K}\left(\mathbb{R}^{n}\right)\right)\right)$. The proof is complete in view of Proposition 7.2 since

$$
H_{\mathrm{alg}}^{*}\left(\mathbb{G}_{K}\left(\mathbb{R}^{n}\right) ; \mathbb{Z} / 2\right)=H^{*}\left(\mathbb{G}_{K}\left(\mathbb{R}^{n}\right) ; \mathbb{Z} / 2\right),
$$

cf. [9, Proposition 11.3.3].

For any real algebraic variety $X$, let $\mathrm{VB}_{\mathbb{R}}^{1}(X)$ denote the group of isomorphism classes of topological $\mathbb{R}$-line bundles on $X$ (with operation induced by tensor product). The first Stiefel-Whitney class gives rise to an isomorphism

$$
w_{1}: \operatorname{VB}_{\mathbb{R}}^{1}(X) \rightarrow H^{1}(X ; \mathbb{Z} / 2) .
$$

Similarly, denote by $\mathrm{VB}_{\mathbb{R} \text {-str }}^{1}(X)$ the group of isomorphism classes of stratified-algebraic $\mathbb{R}$-line bundles on $X$. Now, the first Stiefel-Whitney class gives rise to a homomorphism

$$
w_{1}: \operatorname{VB}_{\mathbb{R}-\mathrm{str}}^{1}(X) \rightarrow H^{1}(X ; \mathbb{Z} / 2) .
$$

Proposition 7.4. For any real algebraic variety $X$,

$$
w_{1}\left(\mathrm{VB}_{\mathbb{R} \text {-str }}^{1}(X)\right)=H_{\text {str }}^{1}(X ; \mathbb{Z} / 2) .
$$

Furthermore, if the variety $X$ is compact, then the homomorphism

$$
w_{1}: \operatorname{VB}_{\mathbb{R}-\text { str }}^{1}(X) \rightarrow H^{1}(X ; \mathbb{Z} / 2)
$$

is injective.

Proof. The inclusion

$$
w_{1}\left(\mathrm{VB}_{\mathbb{R} \text {-str }}^{1}(X)\right) \subseteq H_{\text {str }}^{1}(X ; \mathbb{Z} / 2)
$$

follows from Proposition 7.3 . If $u$ is a cohomology class in $H_{\mathrm{str}}^{1}(X ; \mathbb{Z} / 2)$, then there exists a stratified-regular map $\varphi: X \rightarrow V$, into a compact nonsingular real algebraic variety $V$, such that $u=\varphi^{*}(v)$ for some cohomology class $v$ in $H_{\mathrm{alg}}^{1}(V ; \mathbb{Z} / 2)$. By $[9$, Theorem 12.4.6], $v$ is of the form $v=w_{1}(\lambda)$ for some algebraic $\mathbb{R}$-line bundle $\lambda$ on $V$. The pullback $\mathbb{R}$-line bundle $\varphi^{*} \lambda$ on $X$ is stratified-algebraic, cf. Proposition 2.6. Since $u=\varphi^{*}\left(w_{1}(\lambda)\right)=w_{1}\left(\varphi^{*} \lambda\right)$, we get

$$
H_{\mathrm{str}}^{1}(X ; \mathbb{Z} / 2) \subseteq w_{1}\left(\mathrm{VB}_{\mathbb{R} \text {-str }}^{1}(X)\right) .
$$

If the variety $X$ is compact, then the canonical homomorphism $\operatorname{VB}_{\mathbb{R}-\text { str }}^{1}(X) \rightarrow \operatorname{VB}_{\mathbb{R}}^{1}(X)$ is injective (cf. Theorem 3.10), and hence the last assertion in the proposition follows. 
Proposition 7.4 can be interpreted as an approximation result for maps into real projective $n$-space $\mathbb{P}^{n}(\mathbb{R})=\mathbb{G}_{1}\left(\mathbb{R}^{n+1}\right)$ with $n \geq 1$.

Proposition 7.5. Let $X$ be a compact real algebraic variety. For a continuous map $f: X \rightarrow \mathbb{P}^{n}(\mathbb{R})$, the following conditions are equivalent:

(a) The map $f$ can be approximated by stratified-regular maps.

(b) The cohomology class $f^{*}\left(u_{n}\right)$ belongs to $H_{\mathrm{str}}^{1}(X ; \mathbb{Z} / 2)$, where $u_{n}$ is a generator of the group $H^{1}\left(\mathbb{P}^{n}(\mathbb{R}) ; \mathbb{Z} / 2\right) \cong \mathbb{Z} / 2$.

Proof. Since $u_{n}=w_{1}\left(\gamma_{1}\left(\mathbb{R}^{n+1}\right)\right)$, we have $f^{*}\left(u_{n}\right)=w_{1}\left(f^{*} \gamma_{1}\left(\mathbb{R}^{n+1}\right)\right)$. Thus, it suffices to combine Theorem 4.10 (with $A=\varnothing$ ) and Proposition 7.4.

Corollary 7.6. Let $X$ be a compact real algebraic variety. For a continuous map $f: X \rightarrow \mathbb{S}^{1}$, the following conditions are equivalent:

(a) The map $f$ can be approximated by stratified-regular maps.

(b) The cohomology class $f^{*}(u)$ belongs to $H_{\mathrm{str}}^{1}(X ; \mathbb{Z} / 2)$, where $u$ is a generator of the group $H^{1}\left(\mathbb{S}^{1} ; \mathbb{Z} / 2\right) \cong \mathbb{Z} / 2$.

Proof. It suffices to apply Proposition 7.5 since $\mathbb{S}^{1}$ is biregularly isomorphic to $\mathbb{P}^{1}(\mathbb{R})$.

We next look for relationships between the groups $H_{\mathrm{str}}^{k}(-; \mathbb{Z} / 2)$ and $H_{\text {alg }}^{k}(-; \mathbb{Z} / 2)$.

Proposition 7.7. If $X$ is a compact nonsingular real algebraic variety, then

$$
H_{\mathrm{str}}^{*}(X ; \mathbb{Z} / 2)=H_{\mathrm{alg}}^{*}(X ; \mathbb{Z} / 2)
$$

Proof. The inclusion

$$
H_{\text {alg }}^{*}(X ; \mathbb{Z} / 2) \subseteq H_{\mathrm{str}}^{*}(X ; \mathbb{Z} / 2)
$$

is obvious. According to [33, Propostion 1.3], if $\varphi: X \rightarrow V$ is a continuous rational map into a compact nonsingular real algebraic variety $V$, then

$$
\varphi^{*}\left(H_{\mathrm{alg}}^{*}(V ; \mathbb{Z} / 2)\right) \subseteq H_{\mathrm{alg}}^{*}(X ; \mathbb{Z} / 2) .
$$

Consequently, the inclusion

$$
H_{\mathrm{str}}^{*}(X ; \mathbb{Z} / 2) \subseteq H_{\mathrm{alg}}^{*}(X ; \mathbb{Z} / 2)
$$

follows since each stratified-regular map from $X$ into $V$ is continuous rational, cf. Proposition 2.2 .

For an arbitrary compact real algebraic variety $X$, the group $H_{\text {str }}^{1}(X ; \mathbb{Z} / 2)$ can be described as follows.

Proposition 7.8. Let $X$ be a compact real algebraic variety. For a cohomology class $u$ in $H^{1}(X ; \mathbb{Z} / 2)$, the following conditions are equivalent:

(a) The cohomology class $u$ belongs to $H_{\mathrm{str}}^{1}(X ; \mathbb{Z} / 2)$. 
(b) There exists a filtration $\mathcal{F}=\left(X_{-1}, X_{0}, \ldots, X_{m}\right)$ of $X$ with $\overline{\mathcal{F}}$ a nonsingular stratification, and for each $i=0, \ldots, m$, there exists a multiblowup $\pi_{i}: X_{i}^{\prime} \rightarrow X_{i}$ over $X_{i-1}$ such that $X_{i}^{\prime}$ is a nonsingular variety and the cohomology class $\pi_{i}^{*}\left(\left.u\right|_{X_{i}}\right)$ belongs to $H_{\mathrm{alg}}^{1}\left(X_{i}^{\prime} ; \mathbb{Z} / 2\right)$. Here $\left.u\right|_{X_{i}}$ is the image of $u$ under the homomorphism $H^{1}(X ; \mathbb{Z} / 2) \rightarrow H^{1}\left(X_{i} ; \mathbb{Z} / 2\right)$ induced by the inclusion map $X_{i} \hookrightarrow X$.

Proof. Let $\mathcal{F}=\left(X_{-1}, X_{0}, \ldots, X_{m}\right)$ be a filtration of $X$ with $\overline{\mathcal{F}}$ a nonsingular stratification, and for each $i=0, \ldots, m$, let $\pi_{i}: X_{i}^{\prime} \rightarrow X_{i}$ be a multiblowup over $X_{i-1}$ such that $X_{i}^{\prime}$ is a nonsingular variety. If $\xi$ is a topological $\mathbb{R}$-line bundle on $X$ with $w_{1}(\xi)=u$, then $w_{1}\left(\left.\xi\right|_{X_{i}}\right)=\left.u\right|_{X_{i}}$ and $w_{1}\left(\pi_{i}^{*}\left(\left.\xi\right|_{X_{i}}\right)\right)=\pi_{i}^{*}\left(\left.u\right|_{X_{i}}\right)$ for $0 \leq i \leq m$. In view of [9, Theorem 12.4.6], the $\mathbb{R}$-line bundle $\pi_{i}^{*}\left(\left.\xi\right|_{X_{i}}\right)$ on $X_{i}^{\prime}$ admits an algebraic structure if and only if the cohomology class $\pi_{i}^{*}\left(\left.u\right|_{X_{i}}\right)$ belongs to $H_{\text {alg }}^{1}\left(X_{i}^{\prime} ; \mathbb{Z} / 2\right)$. Consequently, it suffices to combine Corollary 5.3 (with $\mathbb{F}=\mathbb{R}$ ) and Propositions 7.4 and 7.7 .

In [19, 25], algebraic cohomology classes are interpreted as obstructions to representing homotopy classes by regular maps. Some results contained in these papers can be strengthened by transferring them to the framework of stratified objects, cf. Theorem 7.9 below. First it is convenient to introduce some notation.

For any $n$-dimensional compact smooth manifold $M$, let $\llbracket M \rrbracket$ denote its fundamental class in $H_{n}(M ; \mathbb{Z} / 2)$. If $M$ is a smooth submanifold of a smooth manifold $N$, let $\llbracket M \rrbracket_{N}$ denote the homology class in $H_{n}(N ; \mathbb{Z} / 2)$ represented by $M$.

Theorem 7.9. Let $Y$ be a real algebraic variety with $H_{\mathrm{str}}^{k}(Y ; \mathbb{Z} / 2) \neq 0$ for some $k \geq 1$. Then there exist a compact connected nonsingular real algebraic variety $X$ and a continuous map $f: X \rightarrow Y$ such that $\operatorname{dim} X=k+1$ and $f$ is not homotopic to any stratifiedregular map.

Proof. Let $w$ be a nonzero cohomology class in $H_{\text {str }}^{k}(Y ; \mathbb{Z} / 2)$. Choose a homology class $\beta$ in $H_{k}(Y ; \mathbb{Z} / 2)$ which satisfies

$$
\langle w, \beta\rangle \neq 0
$$

and is represented by a singular cycle with support contained in one connected component of $Y$. By [19, Theorem 2.1], $\beta$ can be expressed as

$$
\beta=h_{*}(\llbracket K \rrbracket),
$$

where $K$ is a $k$-dimensional compact connected smooth manifold that is the boundary of a compact smooth manifold with boundary and $h: K \rightarrow Y$ is a continuous map. Let $p_{0}$ be a point in $\mathbb{S}^{1}$. Since the normal bundle of $K \times\left\{p_{0}\right\}$ in $K \times \mathbb{S}^{1}$ is trivial, according to [19, Theorem 2.6, Proposition 2.5], there exist a nonsingular real algebraic variety $X$ and a smooth diffeomorphism $\varphi: X \rightarrow K \times \mathbb{S}^{1}$ such that the homology class $\alpha:=\llbracket \varphi^{-1}\left(K \times\left\{p_{0}\right\}\right) \rrbracket_{X}$ satisfies

$$
\langle u, \alpha\rangle=0
$$

for every cohomology class $u$ in $H_{\text {alg }}^{k}(X ; \mathbb{Z} / 2)$.

Let $\pi: K \times \mathbb{S}^{1} \rightarrow K$ be the canonical projection. It suffices to prove that the continuous map

$$
f:=h \circ \pi \circ \varphi: X \rightarrow Y
$$

is not homotopic to any regular map. This can be done as follows. We have $w=\psi^{*}(v)$ for some stratified-regular map $\psi: Y \rightarrow W$ into a compact nonsingular real algebraic variety 
$V$ and a cohomology class $v$ in $H_{\mathrm{alg}}^{k}(V ; \mathbb{Z} / 2)$. Since $(\pi \circ \varphi)_{*}(\alpha)=\pi_{*}\left(\varphi_{*}(\alpha)\right)=\llbracket K \rrbracket$, we get

$$
(\psi \circ f)_{*}(\alpha)=(\psi \circ h \circ \pi \circ \varphi)_{*}(\alpha)=\psi_{*}\left(h_{*}(\llbracket K \rrbracket)\right)=\psi_{*}(\beta) .
$$

Consequently,

$$
\left\langle(\psi \circ f)^{*}(v), \alpha\right\rangle=\left\langle v,(\psi \circ f)_{*}(\alpha)\right\rangle=\left\langle v, \psi_{*}(\beta)\right\rangle=\left\langle\psi^{*}(v), \beta\right\rangle=\langle w, \beta\rangle \neq 0 .
$$

It follows that the cohomology class $(\psi \circ f)^{*}(v)$ does not belong to $H_{\text {alg }}^{k}(X ; \mathbb{Z} / 2)$. However, if the map $f: X \rightarrow Y$ were homotopic to a stratified-regular map $g: X \rightarrow Y$, then $\psi \circ f$ would be homotopic to a stratified-regular map $\psi \circ g$, and hence the cohomology class $(\psi \circ f)^{*}(v)=(\psi \circ g)^{*}(v)$ would be in $H_{\text {alg }}^{k}(X ; \mathbb{Z} / 2)$, cf. Propositions 7.2 and 7.7. This completes the proof.

We conclude this section by giving an example of an $\mathbb{F}$-line bundle not admitting a stratified-algebraic structure. Recall that $d(\mathbb{F})=\operatorname{dim}_{\mathbb{R}} \mathbb{F}$ and $\mathbb{T}^{n}=\mathbb{S}^{1} \times \cdots \times \mathbb{S}^{1}$ (the $n$-fold product).

Example 7.10. For any integer $n>d(\mathbb{F})$, there exist a nonsingular real algebraic variety $X$ and a topological $\mathbb{F}$-line bundle $\xi$ on $X$ such that $X$ is diffeomorphic to $\mathbb{T}^{n}$ and $\xi$ does not admit a stratified-algebraic structure. This assertion can be proved as follows. Let $d:=d(\mathbb{F})$ and let $y_{0}$ be a point in $\mathbb{T}^{n-d}$. Let $\alpha$ be the homology class in $H_{d}\left(\mathbb{T}^{n} ; \mathbb{Z} / 2\right)$ represented by the smooth submanifold $K:=\mathbb{T}^{d} \times\left\{y_{0}\right\}$ of $\mathbb{T}^{n}$. Set

$$
A:=\left\{u \in H^{d}\left(\mathbb{T}^{n} ; \mathbb{Z} / 2\right) \mid\langle u, \alpha\rangle=0\right\}
$$

Since the normal bundle of $K$ in $\mathbb{T}^{n}$ is trivial and $K$ is the boundary of a compact smooth manifold with boundary, it follows from [19, Propositon 2.5, Theorem 2.6] that there exist a nonsingular real algebraic variety $X$ and a smooth diffeomorphism $\varphi: X \rightarrow \mathbb{T}^{n}$ with

$$
H_{\text {alg }}^{d}(X ; \mathbb{Z} / 2) \subseteq \varphi^{*}(A)
$$

For the $\mathbb{F}$-line bundle $\gamma_{1}\left(\mathbb{F}^{2}\right)$ on $\mathbb{G}_{1}\left(\mathbb{F}^{2}\right)$, one has $w_{d}\left(\gamma_{1}\left(\mathbb{F}^{2}\right)_{\mathbb{R}}\right) \neq 0$ in $H^{d}\left(\mathbb{G}_{1}\left(\mathbb{F}^{2}\right) ; \mathbb{Z} / 2\right)$. Choosing a continuous map $h: \mathbb{T}^{d} \rightarrow \mathbb{G}_{1}\left(\mathbb{F}^{2}\right)$ for which the induced homomorphism

$$
h^{*}: H^{d}\left(\mathbb{G}_{1}\left(\mathbb{F}^{2}\right) ; \mathbb{Z} / 2\right) \rightarrow H^{d}\left(\mathbb{T}^{d} ; \mathbb{Z} / 2\right)
$$

is an isomorphism, we obtain a topological $\mathbb{F}$-line bundle $\lambda:=h^{*} \gamma_{1}\left(\mathbb{F}^{2}\right)$ with $w_{d}\left(\lambda_{\mathbb{R}}\right) \neq 0$ in $H^{d}\left(\mathbb{T}^{d} ; \mathbb{Z} / 2\right)$. If $p: \mathbb{T}^{n}=\mathbb{T}^{d} \times \mathbb{T}^{n-d} \rightarrow \mathbb{T}^{d}$ is the canonical projection and $\eta:=p^{*} \lambda$, then

$$
w_{d}\left(\eta_{\mathbb{R}}\right) \notin A .
$$

Consequently, for the $\mathbb{F}$-line bundle $\xi:=\varphi^{*} \eta$ on $X$, we have

$$
w_{d}\left(\xi_{\mathbb{R}}\right) \notin H_{\mathrm{alg}}^{d}(X ; \mathbb{Z} / 2) .
$$

In view of Propositions 7.3 and 7.7 , the $\mathbb{R}$-vector bundle $\xi_{\mathbb{R}}$ cannot admit a stratifiedalgebraic structure. Hence $\xi$ does not admit a stratified-algebraic structure. 


\section{Stratified- $\mathbb{C}$-algebraic cohomology classes}

We first recall the construction of $\mathbb{C}$-algebraic cohomology classes. Let $V$ be a compact nonsingular real algebraic variety. A nonsingular projective complexification of $V$ is a pair $(\mathbb{V}, \iota)$, where $\mathbb{V}$ is a nonsingular projective scheme over $\mathbb{R}$ and $\iota: V \rightarrow \mathbb{V}(\mathbb{C})$ is an injective map such that $\mathbb{V}(\mathbb{R})$ is Zariski dense in $\mathbb{V}, \iota(V)=\mathbb{V}(\mathbb{R})$ and $\iota$ induces a biregular isomorphism between $V$ and $\mathbb{V}(\mathbb{R})$. Here the set $\mathbb{V}(\mathbb{R})$ of real points of $\mathbb{V}$ is regarded as a subset of the set $\mathbb{V}(\mathbb{C})$ of complex points of $\mathbb{V}$. The existence of $(\mathbb{V}, \iota)$ follows from Hironaka's theorem on resolution of singularities [27, 31]. We identify $\mathbb{V}(\mathbb{C})$ with the set of complex points of the scheme $\mathbb{V}_{\mathbb{C}}:=\mathbb{V} \times_{\text {Spec } \mathbb{R}}$ Spec $\mathbb{C}$ over $\mathbb{C}$. The cycle map

$$
\operatorname{cl}_{\mathbb{C}}: A^{*}\left(\mathbb{V}_{\mathbb{C}}\right)=\bigoplus_{k \geq 0} A^{k}\left(\mathbb{V}_{\mathbb{C}}\right) \rightarrow H^{\text {even }}(\mathbb{V}(\mathbb{C}) ; \mathbb{Z})=\bigoplus_{k \geq 0} H^{2 k}(\mathbb{V}(\mathbb{C}) ; \mathbb{Z})
$$

is a ring homomorphism defined on the Chow ring of $\mathbb{V}_{\mathbb{C}}$, cf. [20] or [24, Corollary 19.2]. Hence

$$
H_{\text {alg }}^{2 k}(\mathbb{V}(\mathbb{C}) ; \mathbb{Z}):=\operatorname{cl}_{\mathbb{C}}\left(A^{k}\left(\mathbb{V}_{\mathbb{C}}\right)\right)
$$

is the subgroup of $H^{2 k}(\mathbb{V}(\mathbb{C}) ; \mathbb{Z})$ that consists of the cohomology classes corresponding to algebraic cycles (defined over $\mathbb{C}$ ) on $\mathbb{V}_{\mathbb{C}}$ of codimension $k$. By construction,

$$
H_{\mathbb{C}-\operatorname{alg}}^{2 k}(V ; \mathbb{Z}):=\iota^{*}\left(H_{\text {alg }}^{2 k}(\mathbb{V}(\mathbb{C}) ; \mathbb{Z})\right)
$$

is a subgroup of $H^{2 k}(V ; \mathbb{Z})$, and

$$
H_{\mathbb{C} \text {-alg }}^{\text {even }}(V ; \mathbb{Z}):=\bigoplus_{k \geq 0} H_{\mathbb{C} \text {-alg }}^{2 k}(V ; \mathbb{Z})
$$

is a subring of $H^{\text {even }}(V ; \mathbb{Z})$. The subring $H_{\mathbb{C} \text {-alg }}^{\text {eve }}(V ; \mathbb{Z})$ does not depend on the choice of $(\mathbb{V}, \iota)$. If the variety $V$ is irreducible, then $H_{\mathbb{C} \text {-alg }}^{0}(V ; \mathbb{Z})$ is the subgroup generated by $1 \in H^{0}(V ; \mathbb{Z})$. A cohomology class $v$ in $H^{2 k}(V ; \mathbb{Z})$ is said to be $\mathbb{C}$-algebraic if it belongs to $H_{\mathbb{C} \text {-alg }}^{2 k}(V ; \mathbb{Z})$. For any algebraic $\mathbb{C}$-vector bundle $\xi$ on $V$, its $k$ th Chern class $c_{k}(\xi)$ is in $H_{\mathbb{C} \text {-alg }}^{2 k}(V ; \mathbb{Z})$ for every $k \geq 0$. If $h: V \rightarrow W$ is a regular map between compact nonsingular real algebraic varieties, then

$$
h^{*}\left(H_{\mathbb{C} \text {-alg }}^{\text {even }}(W ; \mathbb{Z})\right) \subseteq H_{\mathbb{C} \text {-alg }}^{\text {even }}(V ; \mathbb{Z}) .
$$

Proofs of these properties of $H_{\mathbb{C}-a l g}^{\text {even }}(-; \mathbb{Z})$ are given in [8]. Numerous applications of $\mathbb{C}$ algebraic cohomology classes can be found in [8, 12, 16, 18, 34.

Let $X$ be a real algebraic variety. A cohomology class $u$ in $H^{2 k}(X ; \mathbb{Z})$ is said to be stratified-C-algebraic if there exists a stratified-regular map $\varphi: X \rightarrow V$, into a compact nonsingular real algebraic variety $V$, such that $u=\varphi^{*}(v)$ for some cohomology class $v$ in $H_{\mathbb{C} \text {-alg }}^{2 k}(V ; \mathbb{Z})$.

Proposition 8.1. For any real algebraic variety $X$, the set $H_{\mathbb{C} \text {-str }}^{2 k}(X ; \mathbb{Z})$ of all stratified$\mathbb{C}$-algebraic cohomology classes in $H^{2 k}(V ; \mathbb{Z})$ forms a subgroup. Furthermore, the direct sum

$$
H_{\mathbb{C} \text {-str }}^{\text {even }}(X ; \mathbb{Z}):=\bigoplus_{k \geq 0} H_{\mathbb{C} \text {-str }}^{2 k}(X ; \mathbb{Z})
$$

is a subring of $H^{\text {even }}(X ; \mathbb{Z})$. 
Proof. It suffices to adapt the proof of Proposition 7.1.

Proposition 8.2. If $f: X \rightarrow Y$ is a stratified-regular map between real algebraic varieties, then

$$
f^{*}\left(H_{\mathbb{C} \text {-str }}^{\text {even }}(Y ; \mathbb{Z})\right) \subseteq H_{\mathbb{C} \text {-str }}^{\text {even }}(X ; \mathbb{Z}) .
$$

Proof. The proof of Proposition 7.2 also works in the case under consideration here.

Proposition 8.3. Let $\xi$ be a stratified-algebraic $\mathbb{C}$-vector bundle on a real algebraic variety $X$. Then the $k$ th Chern class $c_{k}(\xi)$ of $\xi$ belongs to $H_{\mathbb{C} \text {-str }}^{2 k}(X ; \mathbb{Z})$ for every $k \geq 0$.

Proof. One can copy the proof of Proposition 7.3. It suffices to observe that

$$
H_{\mathbb{C} \text {-alg }}^{\text {even }}\left(\mathbb{G}_{K}\left(\mathbb{C}^{n}\right) ; \mathbb{Z}\right)=H^{\text {even }}\left(\mathbb{G}_{K}\left(\mathbb{C}^{n}\right) ; \mathbb{Z}\right),
$$

cf. [34, Example 5.5].

Corollary 8.4. Let $\xi$ be a stratified-regular $\mathbb{R}$-vector bundle on a real algebraic variety $X$. Then the $k$ th Pontryagin class $p_{k}(\xi)$ of $\xi$ belongs to $H_{\mathbb{C}-\text { str }}^{4 k}(X ; \mathbb{Z})$ for every $k \geq 0$.

Proof. Since $p_{k}(\xi)=(-1)^{k} c_{2 k}(\mathbb{C} \otimes \xi)$, is suffices to make use of Proposition 8.3.

For any topological $\mathbb{F}$-vector bundle $\xi$ on $X$, one can interpret rank $\xi$ as an element of $H^{0}(X ; \mathbb{Z})$. Then the following holds.

Proposition 8.5. Let $X$ be a real algebraic variety. If $\xi$ is a stratified-algebraic $\mathbb{F}$-vector bundle on $X$, then rank $\xi$ belongs to $H_{\mathbb{C}-s t r}^{0}(X ; \mathbb{Z})$. Conversely, each cohomology class in $H_{\mathbb{C} \text {-str }}^{0}(X ; \mathbb{Z})$ is of the form rank $\eta$ for some stratified-algebraic $\mathbb{F}$-vector bundle $\eta$ on $X$, whose restriction to each connected component of $X$ is topologically trivial.

Proof. By Proposition 3.4. $\xi$ is of the form $\xi=f^{*} \gamma_{K}\left(\mathbb{F}^{n}\right)$ for some multi-Grassmannian $\mathbb{G}_{K}\left(\mathbb{F}^{n}\right)$ and stratified-regular map $f: X \rightarrow \mathbb{G}_{K}\left(\mathbb{F}^{n}\right)$. Since rank $\xi=f^{*}\left(\operatorname{rank} \gamma_{K}\left(\mathbb{F}^{n}\right)\right)$ and

$$
H_{\mathbb{C} \text {-alg }}^{0}\left(\mathbb{G}_{K}\left(\mathbb{F}^{n}\right) ; \mathbb{Z}\right)=H^{0}\left(\mathbb{G}_{K}\left(\mathbb{F}^{n}\right) ; \mathbb{Z}\right),
$$

it follows that rank $\xi$ belongs to $H_{\mathbb{C} \text {-str }}^{0}(X ; \mathbb{Z})$. The second part of the proposition readily follows from the description of $H_{\mathbb{C}-s t r}^{0}(X ; \mathbb{Z})$.

For any real algebraic variety $X$, let $\operatorname{VB}_{\mathbb{C}}^{1}(X)$ denote the group of isomorphism classes of topological $\mathbb{C}$-line bundles on $X$ (with operation induced by tensor product). The first Chern class gives rise to an isomorphism

$$
c_{1}: \operatorname{VB}_{\mathbb{C}}^{1}(X) \rightarrow H^{2}(X ; \mathbb{Z}) .
$$

Similarly, denote by $\mathrm{VB}_{\mathbb{C}-\text { str }}^{1}(X)$ the group of isomorphism classes of stratified-algebraic $\mathbb{C}$-line bundles on $X$. Now, the first Chern class gives rise to a homomorphism

$$
c_{1}: \operatorname{VB}_{\mathbb{C}-\text { str }}^{1}(X) \rightarrow H^{2}(X ; \mathbb{Z}) .
$$

Proposition 8.6. For any real algebraic variety $X$,

$$
c_{1}\left(\mathrm{VB}_{\mathbb{C} \text {-str }}^{1}(X)\right)=H_{\mathbb{C} \text {-str }}^{2}(X ; \mathbb{Z}) .
$$

Furthermore, if the variety $X$ is compact, then the homomorphism

$$
c_{1}: \mathrm{VB}_{\mathbb{C} \text {-str }}^{1}(X) \rightarrow H^{2}(X ; \mathbb{Z})
$$

is injective. 
Proof. The inclusion

$$
c_{1}\left(\mathrm{VB}_{\mathbb{C} \text {-str }}^{1}(X)\right) \subseteq H_{\mathbb{C}-\text { str }}^{2}(X ; \mathbb{Z})
$$

follows from Proposition 8.3. If $u$ is a cohomology class in $H_{\mathbb{C} \text {-str }}^{2}(X ; \mathbb{Z})$, then there exists a stratified-regular map $\varphi: X \rightarrow V$, into a compact nonsingular real algebraic variety $V$, such that $u=\varphi^{*}(v)$ for some cohomology class $v$ in $H_{\mathbb{C} \text {-alg }}^{2}(V ; \mathbb{Z})$. By [8, Remark 5.4], $v$ is of the form $v=c_{1}(\lambda)$ for some algebraic $\mathbb{C}$-line bundle $\lambda$ on $V$. The pullback $\mathbb{C}$-line bundle $\varphi^{*} \lambda$ on $X$ is stratified-algebraic, cf. Proposition 2.6. Since $u=\varphi^{*}\left(c_{1}(\lambda)\right)=c_{1}\left(\varphi^{*} \lambda\right)$, we get

$$
H_{\mathbb{C} \text {-str }}^{2}(X ; \mathbb{Z}) \subseteq c_{1}\left(\operatorname{VB}_{\mathbb{C} \text {-str }}^{1}(X)\right) .
$$

If the variety $X$ is compact, then the canonical homomorphism $\operatorname{VB}_{\mathbb{C} \text {-str }}^{1}(X) \rightarrow \operatorname{VB}_{\mathbb{C}}^{1}(X)$ is injective (cf. Theorem 3.10), and hence the last assertion in the proposition follows.

Proposition 8.6 can be interpreted as an approximation result for maps into complex projective $n$-space $\mathbb{P}^{n}(\mathbb{C})=\mathbb{G}_{1}\left(\mathbb{C}^{n+1}\right)$ for $n \geq 1$.

Proposition 8.7. Let $X$ be a compact real algebraic variety. For a continuous map $f: X \rightarrow \mathbb{P}^{n}(\mathbb{C})$, the following conditions are equivalent:

(a) The map $f$ can be approximated by stratified-regular maps.

(b) The cohomology class $f^{*}\left(v_{n}\right)$ belongs to $H_{\mathbb{C}-\text { str }}^{2}(X ; \mathbb{Z})$, where $v_{n}$ is a generator of the group $H^{2}\left(\mathbb{P}^{n}(\mathbb{C}) ; \mathbb{Z}\right) \cong \mathbb{Z}$.

Proof. We may assume that $v_{n}=c_{1}\left(\gamma_{1}\left(\mathbb{C}^{n+1}\right)\right)$. Since $f^{*}\left(v_{n}\right)=c_{1}\left(f^{*} \gamma_{1}\left(\mathbb{C}^{n+1}\right)\right)$, it suffices to make use of Theorem 4.10 (with $A \neq \varnothing$ ) and Proposition 8.6 .

Corollary 8.8. Let $X$ be a compact real algebraic variety. For a continuous map $f: X \rightarrow \mathbb{S}^{2}$, the following conditions are equivalent:

(a) The map $f$ can be approximated by stratified-regular maps.

(b) The cohomology class $f^{*}\left(s_{2}\right)$ belongs to $H_{\mathbb{C}-\text { str }}^{2}(X ; \mathbb{Z})$, where $s_{2}$ is a generator of the group $H^{2}\left(\mathbb{S}^{2} ; \mathbb{Z}\right) \cong \mathbb{Z}$.

Proof. It suffices to apply Proposition 8.7 since $\mathbb{S}^{2}$ is biregularly isomorphic to $\mathbb{P}^{1}(\mathbb{C})$.

Any real algebraic variety $X$ is homotopically equivalent to a compact polyhedron 9 , Corollary 9.6.7], and hence the Chern character

$$
\operatorname{ch}: K_{\mathbb{C}}(X) \rightarrow H^{\text {even }}(X ; \mathbb{Q})
$$

induces an isomorphism

$$
\operatorname{ch}: K_{\mathbb{C}}(X) \otimes \mathbb{Q} \rightarrow H^{\text {even }}(X ; \mathbb{Q}),
$$

cf. [4] or [26, p. 255, Theorem A]. On the other hand, the Chern character

$$
\operatorname{ch}: K_{\mathbb{C}-\text { str }}(X) \rightarrow H^{\text {even }}(X ; \mathbb{Q})
$$

induces a homomorphism

$$
\operatorname{ch}: K_{\mathbb{C} \text {-str }}(X) \otimes \mathbb{Q} \rightarrow H^{\text {even }}(X ; \mathbb{Q}) .
$$

We next describe the image of the last homomorphism. Denote by $H_{\mathbb{C} \text {-str }}^{\text {even }}(X ; \mathbb{Q})$ the image of $H_{\mathbb{C} \text {-str }}^{\text {even }}(X ; \mathbb{Z}) \otimes \mathbb{Q}$ by the canonical isomorphism $H^{\text {even }}(X ; \mathbb{Z}) \otimes \mathbb{Q} \rightarrow H^{\text {even }}(X ; \mathbb{Q})$. 
Proposition 8.9. For any real algebraic variety $X$,

$$
\operatorname{ch}\left(K_{\mathbb{C}-\text { str }}(X) \otimes \mathbb{Q}\right)=H_{\mathbb{C}-\text { str }}^{\text {even }}(X ; \mathbb{Q}) .
$$

Furthermore, if the variety $X$ is compact, then the homomorphism

$$
\operatorname{ch}: K_{\mathbb{C}-\text { str }}(X) \otimes \mathbb{Q} \rightarrow H^{\text {even }}(X ; \mathbb{Q})
$$

is injective.

Proof. The inclusion

$$
\operatorname{ch}\left(K_{\mathbb{C} \text {-str }}(X) \otimes \mathbb{Q}\right) \subseteq H_{\mathbb{C} \text {-str }}^{\text {even }}(X ; \mathbb{Q})
$$

follows from Propositions 8.3 and 8.5 . If $u$ is a cohomology class in $H_{\mathbb{C}-\text { str }}^{\text {even }}(X ; \mathbb{Q})$, then there exists a stratified-regular map $\varphi: X \rightarrow V$, into a compact nonsingular real algebraic variety $V$, such that $u=\varphi^{*}(v)$ for some cohomology class $v$ in $H_{\mathbb{C} \text {-alg }}^{\text {even }}(V ; \mathbb{Q})$. By $[8$, Proposition 5.4], $v$ is of the form $v=\operatorname{ch}(\alpha)$ for some element $\alpha$ in $K_{\mathbb{C} \text {-alg }}(X) \otimes \mathbb{Q}$. In view of Proposition 2.6, $\varphi^{*}(\alpha)$ is in $K_{\mathbb{C} \text {-str }}(X) \otimes \mathbb{Q}$. Since $u=\varphi^{*}(\operatorname{ch}(\alpha))=\operatorname{ch}\left(\varphi^{*}(\alpha)\right)$, we get

$$
H_{\mathbb{C}-\text { str }}^{\text {even }}(X ; \mathbb{Q}) \subseteq \operatorname{ch}\left(K_{\mathbb{C} \text {-str }}(X) \otimes \mathbb{Q}\right)
$$

If the variety $X$ is compact, then the canonical homomorphism $K_{\mathbb{C} \text {-str }}(X) \rightarrow K_{\mathbb{C}}(X)$ is injective (cf. Corollary 3.11), and hence the last assertion in the proposition follows.

One can also prove a sharper result than the first part of Proposition 8.9.

Proposition 8.10. Let $X$ be a real algebraic variety and let $k$ be a positive integer. For any cohomology class $u$ in $H_{\mathbb{C} \text {-str }}^{2 k}(X ; \mathbb{Z})$, there exists a stratified-algebraic $\mathbb{C}$-vector bundle $\xi$ on $X$ with $c_{i}(\xi)=0$ for $1 \leq i \leq k-1$ and $c_{k}(\xi)=(-1)^{k-1}(k-1) ! u$.

Proof. We first consider $\mathbb{C}$-algebraic cohomology classes.

Assertion. Let $V$ be a compact nonsingular real algebraic variety and let $v$ be a cohomology class in $H_{\mathbb{C} \text {-alg }}^{2 k}(V ; \mathbb{Z})$, where $k \geq 1$. Then there exists an algebraic $\mathbb{C}$-vector bundle $\eta$ on $V$ with $c_{i}(\eta)=0$ for $1 \leq i \leq k-1$ and $c_{k}(\eta)=(-1)^{k-1}(k-1) ! v$.

The Assertion can be proved as follows. Let $(\mathbb{V}, \iota)$ be a nonsingular projective complexification of $V$. We may assume that $V=\mathbb{V}(\mathbb{R})$ and $\iota: V \hookrightarrow \mathbb{V}(\mathbb{C})$ is the inclusion map. By definition, $v=\iota^{*}\left(\operatorname{cl}_{\mathbb{C}}(z)\right)$ for some element $z$ in the Chow group $A^{k}\left(\mathbb{V}_{\mathbb{C}}\right)$. Let $\mathbb{U}$ be an affine Zariski open subscheme of $\mathbb{V}$ containing $V$. According to Grothendieck's formula [24, Example 15.3] (see also [24, Propostition 19.1.2]), there exists an algebraic vector bundle $\mathbb{E}$ on $\mathbb{U}_{\mathbb{C}}$ with $c_{i}(\mathbb{E})=0$ for $1 \leq i \leq k-1$ and $c_{k}(\mathbb{E})=(-1)^{k-1}(k-1) ! j^{*}\left(\operatorname{cl}_{\mathbb{C}}(z)\right)$, where $j: \mathbb{U}(\mathbb{C}) \rightarrow \mathbb{V}(\mathbb{C})$ is the inclusion map. The restriction $\eta:=\left.\mathbb{E}\right|_{V}$ is an algebraic $\mathbb{C}$-vector bundle on $V$ satisfying all the conditions stated in the Assertion.

We can now easily complete the proof. The cohomology class $u$ is of the form $u=\varphi^{*}(v)$, where $\varphi: X \rightarrow V$ is a stratified-regular map into a compact nonsingular real algebraic variety $V$, and $v$ is a cohomology class in $H_{\mathbb{C} \text {-alg }}^{2 k}(V ; \mathbb{Z})$. If $\eta$ is an algebraic $\mathbb{C}$-vector bundle on $V$ as in the Assertion, then the pullback $\varphi^{*} \eta$ is a stratified-algebraic $\mathbb{C}$-vector bundle on $X$ (cf. Proposition 2.6) having all the required properties.

Under some assumptions we obtain a nice characterization of topological $\mathbb{C}$-vector bundles admitting a stratified-algebraic structure. 
Theorem 8.11. Let $X$ be a compact real algebraic variety. Assume that the group $H^{*}(X ; \mathbb{Z})$ has no torsion and the quotient group $H^{2 k}(X ; \mathbb{Z}) / H_{\mathbb{C} \text {-str }}^{2 k}(X ; \mathbb{Z})$ has no $(k-1)$ !torsion elements for every $k \geq 1$. For a topological $\mathbb{C}$-vector bundle $\xi$ on $X$, the following conditions are equivalent:

(a) $\xi$ admits a stratified-algebraic structure.

(b) $\operatorname{rank} \xi$ belongs to $H_{\mathbb{C} \text {-str }}^{0}(X ; \mathbb{Z})$, and $c_{k}(\xi)$ belongs to $H_{\mathbb{C} \text {-str }}^{2 k}(X ; \mathbb{Z})$ for every $k \geq 1$.

Proof. According to Propositions 8.3 and 8.5 , (a) implies (b). For the proof of the reversed implication, we first establish the following:

Assertion. Let $\theta$ be a topological $\mathbb{C}$-vector bundle on $X$ and let $k$ be a positive integer such that $c_{i}(\theta)=0$ for $1 \leq i \leq k-1$ and $c_{k}(\theta)$ belongs to $H_{\mathbb{C}-s t r}^{2 k}(X ; \mathbb{Z})$. Then there exists a stratified-algebraic $\mathbb{C}$-vector bundle $\theta_{k}$ on $X$ with $c_{i}\left(\theta_{k}\right)=0$ for $1 \leq i \leq k-1$ and $c_{k}\left(\theta \oplus \theta_{k}\right)=0$.

Recall that $X$ is a compact polyhedron, cf. [9, Corollary 9.6.7]. Since the group $H^{*}(X ; \mathbb{Z})$ has no torsion, the $k$ th Chern class $c_{k}(\theta)$ is of the form

$$
c_{k}(\theta)=(-1)^{k-1}(k-1) ! u
$$

for some cohomology class $u$ in $H^{2 k}(X ; \mathbb{Z})$, cf. [4, p. 19]. By assumption, $(-1)^{k-1}(k-1) ! u$ is in $H_{\mathbb{C} \text {-str }}^{2 k}(X ; \mathbb{Z})$, and hence $u$ is in $H_{\mathbb{C} \text {-str }}^{2 k}(X ; \mathbb{Z})$, the quotient group

$$
H^{2 k}(X ; \mathbb{Z}) / H_{\mathbb{C} \text {-str }}^{2 k}(X ; \mathbb{Z})
$$

having no $(k-1)$ !-torsion elements. According to Proposition 8.10, there exists a stratifiedalgebraic $\mathbb{C}$-vector bundle $\theta_{k}$ on $X$ with $c_{i}\left(\theta_{k}\right)=0$ for $1 \leq i \leq k-1$ and $c_{k}\left(\theta_{k}\right)=$ $(-1)^{k-1}(k-1) !(-u)$. Thus, $\theta_{k}$ satisfies all the conditions stated in the Assertion.

If (b) holds, then making use of the Assertion, we obtain a stratified-algebraic $\mathbb{C}$-vector bundle $\zeta$ on $X$ with $c_{i}(\xi \oplus \zeta)=0$ for every $i \geq 1$. If $\eta$ is a stratified-algebraic $\mathbb{C}$-vector bundle on $X$ such that the direct sum $\zeta \oplus \eta$ is a trivial vector bundle (cf. Proposition 3.7), then $c_{i}(\xi)=c_{i}(\eta)$ for every $i \geq 1$. Now, in view of the second part of Proposition 8.5. there exists a stratified-algebraic $\mathbb{C}$-vector bundle $\xi^{\prime}$ on $X$ such that rank $\xi-\operatorname{rank} \xi^{\prime}$ is constant, and $c_{i}(\xi)=c_{i}\left(\xi^{\prime}\right)$ for every $i \geq 1$. Consequently, the $\mathbb{C}$-vector bundles $\xi$ and $\xi^{\prime}$ are topologically stably equivalent, the group $H^{\text {even }}(X ; \mathbb{Z})$ having no torsion, cf. [41]. Hence, according to Corollary 3.14, $\xi$ admits a stratified-algebraic structure.

Any $\mathbb{H}$-vector bundle $\xi$ can be regarded as a $\mathbb{C}$-vector bundle, which is indicated by $\xi_{\mathbb{C}}$.

Corollary 8.12. Let $X$ be a compact real algebraic variety. Assume that the group $H^{*}(X ; \mathbb{Z})$ has no torsion and the quotient group $H^{2 k}(X ; \mathbb{Z}) / H_{\mathbb{C} \text {-str }}^{2 k}(X ; \mathbb{Z})$ has no $(k-1)$ !torsion elements for every $k \geq 1$. For a topological $\mathbb{H}$-vector bundle $\xi$ on $X$, the following conditions are equivalent:

(a) $\xi$ admits a stratified-algebraic structure.

(b) rank $\xi$ belongs to $H_{\mathbb{C}-\text { str }}^{0}(X ; \mathbb{Z})$, and $c_{2 k}\left(\xi_{\mathbb{C}}\right)$ belongs to $H_{\mathbb{C} \text {-str }}^{4 k}(X ; \mathbb{Z})$ for every $k \geq 1$.

Proof. According to Propositions 8.3 and 8.5, (a) implies (b). Since $\xi$ is an $\mathbb{H}$-vector bundle, it follows that $c_{2 i+1}(\xi)=0$ for every $i \geq 0$. Hence, in view of Theorem 8.11 . if (b) holds, then the $\mathbb{C}$-vector bundle $\xi_{\mathbb{C}}$ admits a stratified-algebraic structure. Consequently, the $\mathbb{R}$-vector bundle $\xi_{\mathbb{R}}=\left(\xi_{\mathbb{C}}\right)_{\mathbb{R}}$ admits a stratified-algebraic structure. Thus, by Theorem 1.7, (b) implies (a). 
For $\mathbb{R}$-vector bundles we can only obtain a weaker result.

Corollary 8.13. Let $X$ be a compact real algebraic variety and let $\xi$ be a topological $\mathbb{R}$ vector bundle on $X$. Assume that the group $H^{*}(X ; \mathbb{Z})$ has no torsion and the quotient group $H^{2 k}(X ; \mathbb{Z}) / H_{\mathbb{C} \text {-str }}^{2 k}(X ; \mathbb{Z})$ has no $(k-1)$ !-torsion elements for every $k \geq 1$. If rank $\xi$ belongs to $H_{\mathbb{C} \text {-str }}^{0}(X ; \mathbb{Z})$, and $p_{k}(\xi)$ belongs to $H_{\mathbb{C} \text {-str }}^{4 k}(X ; \mathbb{Z})$ for every $k \geq 1$, then the direct sum $\xi \oplus \xi$ admits a stratified-algebraic structure.

Proof. We have $c_{2 i+1}(\mathbb{C} \otimes \xi)=0$ for every $i \geq 0$ since $c_{2 i+1}(\mathbb{C} \otimes \xi)$ is always an element of order at most 2 (cf. [39, p. 174]) and the group $H^{\text {even }}(X ; \mathbb{Z})$ has no torsion. Moreover, $c_{2 k}(\mathbb{C} \otimes \xi)=(-1)^{k} p_{k}(\xi)$ for every $k \geq 1$. According to Theorem 8.11, the $\mathbb{C}$-vector bundle $\mathbb{C} \otimes \xi$ admits a stratified-algebraic structure. The proof is complete since $(\mathbb{C} \otimes \xi)_{\mathbb{R}}=\xi \oplus \xi$.

We next identify some classes of real algebraic varieties which satisfy the assumptions of the last three results.

Lemma 8.14. If $X$ is a compact real algebraic variety of even dimension $2 k$, then

$$
H_{\mathbb{C}-\text { str }}^{2 k}(X ; \mathbb{Z})=H^{2 k}(X ; \mathbb{Z}) .
$$

Proof. Let $s_{2 k}$ be a generator of the cohomology group $H^{2 k}\left(\mathbb{S}^{2 k} ; \mathbb{Z}\right) \cong \mathbb{Z}$. Each cohomology class $u$ in $H^{2 k}(X ; \mathbb{Z})$ is of the form $u=f^{*}\left(s_{2 k}\right)$ for some continuous map $f: X \rightarrow \mathbb{S}^{2 k}$. According to Theorem 2.5, we may assume that $f$ is stratified-regular. The proof is complete since $H_{\mathbb{C} \text {-alg }}^{2 k}\left(\mathbb{S}^{2 k} ; \mathbb{Z}\right)=H^{2 k}\left(\mathbb{S}^{2 k} ; \mathbb{Z}\right)$, cf. [8, Propostion 4.8].

Proposition 8.15. Let $X=X_{1} \times \cdots \times X_{n}$, where each $X_{i}$ is a compact real algebraic variety homotopically equivalent to the unit $d_{i}$-sphere for $1 \leq i \leq n$. Then

$$
H_{\mathbb{C} \text {-str }}^{\text {even }}(X ; \mathbb{Z})=H^{\text {even }}(X ; \mathbb{Z}) .
$$

Proof. Let $p_{i}: X \rightarrow X_{i}$ be the canonical projection, $1 \leq i \leq n$. For each pair $(j, l)$ of integers satisfying $1 \leq j \leq l \leq n$, let $q_{j l}: X \rightarrow X_{j} \times X_{l}$ be the canonical projection. The $\mathbb{Z}$-algebra $H^{\text {even }}(X ; \mathbb{Z})$ is generated by all $p_{i}^{*}\left(H^{d_{i}}\left(X_{i} ; \mathbb{Z}\right)\right)$ with $d_{i}$ even and all $q_{j l}^{*}\left(H^{d_{j}+d_{l}}\left(X_{j} \times X_{l} ; \mathbb{Z}\right)\right)$ with $d_{j}$ and $d_{l}$ odd. The proof is complete in view of Lemma 8.14 and Propositions 8.1 and 8.2 .

The reader may compare Proposition 8.15 and results of [14, 16, 18] to see a sharp difference between $H_{\mathbb{C} \text {-str }}^{\text {even }}(-; \mathbb{Z})$ and $H_{\mathbb{C} \text {-alg }}^{\text {even }}(-; \mathbb{Z})$.

Proof of Theorem 1.10. It suffices to combine Theorem 8.11, Corollaries 8.12, 8.13, and Proposition 8.15 .

Theorem 1.10 can be interpreted as an approximation result for maps into Grassmannians.

Theorem 8.16. Let $X=X_{1} \times \cdots X_{n}$, where each $X_{i}$ is a compact real algebraic variety homotopically equivalent to the unit $d_{i}$-sphere for $1 \leq i \leq n$. If $\mathbb{F}=\mathbb{C}$ or $\mathbb{F}=\mathbb{H}$, then for any pair $(k, m)$ of integers satisfying $1 \leq k \leq m$, each continuous map from $X$ into the Grassmannian $\mathbb{G}_{k}\left(\mathbb{F}^{m}\right)$ can be approximated by stratified-regular maps.

Proof. If suffices to make use of Theorem 1.10 and Theorem 4.10 (with $A=\varnothing$ ). 
As an interesting special case, we obtain the following approximation result for maps with values in the unit spheres $\mathbb{S}^{2}$ or $\mathbb{S}^{4}$.

Corollary 8.17. Let $X=X_{1} \times \cdots \times X_{n}$, where each $X_{i}$ is a compact real algebraic variety homotopically equivalent to the unit $d_{i}$-sphere for $1 \leq i \leq n$. If $k=2$ or $k=4$, then each continuous map from $X$ into the unit $k$-sphere $\mathbb{S}^{k}$ can be approximated by stratified-regular maps.

Proof. Recall that $d(\mathbb{F})=\operatorname{dim}_{\mathbb{R}} \mathbb{F}$, and $\mathbb{G}_{1}\left(\mathbb{F}^{2}\right)$ is biregularly isomorphic to $\mathbb{S}^{d(\mathbb{F})}$. Thus, Corollary 8.17 is a special case of Theorem 8.16 .

We also have the following result analogous to Proposition 8.15.

Theorem 8.18. Let $X$ be a compact real algebraic variety with

$$
H_{\mathrm{str}}^{1}(X ; \mathbb{Z} / 2)=H^{1}(X ; \mathbb{Z} / 2)
$$

Assume that for a positive integer $k$, the cohomology group $H^{2 k}(X ; \mathbb{Z})$ is generated by the cup products of cohomology classes belonging to $H^{1}(X ; \mathbb{Z})$. Then

$$
H_{\mathbb{C} \text {-str }}^{2 k}(X ; \mathbb{Z})=H^{2 k}(X ; \mathbb{Z}) .
$$

Proof. Each cohomology class in $H^{1}(X ; \mathbb{Z})$ is of the form $f^{*}\left(s_{1}\right)$, where $f: X \rightarrow \mathbb{S}^{1}$ is a continuous map and $s_{1}$ is a generator of the group $H^{1}(X ; \mathbb{Z}) \cong \mathbb{Z}$, cf. [43, pp. 425, 428]. Consequently, the group $H^{2 k}(X ; \mathbb{Z})$ is generated by the cohomology classes of the form $g^{*}\left(t_{2 k}\right)$, where $g$ is a continuous map from $X$ into the $2 k$-torus $\mathbb{T}^{2 k}=\mathbb{S}^{1} \times \cdots \times \mathbb{S}^{1}$, and $t_{2 k}$ is a generator of the group $H^{2 k}\left(\mathbb{T}^{2 k} ; \mathbb{Z}\right) \cong \mathbb{Z}$. In view of Corollary 7.6 and the equality $H_{\text {str }}^{1}(X ; \mathbb{Z} / 2)=H^{1}(X ; \mathbb{Z} / 2)$, we may assume that the map $g$ is stratified-regular. By Proposition 8.15, $H_{\mathbb{C} \text {-str }}^{2 k}\left(\mathbb{T}^{2 k} ; \mathbb{Z}\right)=H^{2 k}\left(\mathbb{T}^{2 k} ; \mathbb{Z}\right)$. In order to complete the proof it suffices to apply Propositions 8.1 and 8.2 .

Corollary 8.19. Let $X$ be a compact real algebraic variety with

$$
H_{\mathrm{str}}^{1}(X ; \mathbb{Z} / 2)=H^{1}(X ; \mathbb{Z} / 2) .
$$

Assume that $X$ is homotopically equivalent to the $n$-torus $\mathbb{S}^{1} \times \cdots \times \mathbb{S}^{1}$ ( $n$ factors). Then

$$
H_{\mathbb{C} \text {-str }}^{\text {even }}(X ; \mathbb{Z})=H^{\text {even }}(X ; \mathbb{Z})
$$

If $\mathbb{F}=\mathbb{C}$ or $\mathbb{F}=\mathbb{H}$, then each topological $\mathbb{F}$-vector bundle on $X$ admits a stratifiedalgebraic structure. If $\xi$ is a topological $\mathbb{R}$-vector bundle on $X$, then the direct sum $\xi \oplus \xi$ admits a stratified-algebraic structure.

Proof. Obviously, $H_{\mathbb{C}-s t r}^{0}(X ; \mathbb{Z})=H^{0}(X ; \mathbb{Z}) \cong \mathbb{Z}$, the group $H^{*}(X ; \mathbb{Z})$ has no torsion, and the $\mathbb{Z}$-algebra $H^{*}(X ; \mathbb{Z})$ is generated by $H^{1}(X ; \mathbb{Z})$. It suffices to apply Theorem 8.18 , Theorem 8.11 and Corollaries 8.12 and 8.13 .

We conclude this section by giving a different description of the cohomology group $H_{\mathbb{C} \text {-str }}^{2}(X ; \mathbb{Z})$, which in turn leads to a new interpretation of our results on $\mathbb{C}$-line bundles. First some topological facts will be recalled for the convenience of the reader.

Let $M$ be a smooth manifold and let $N$ be a smooth submanifold of $M$ of codimension $k$. By convention, submanifolds are assumed to be closed subsets of the ambient manifold. Assume that the normal bundle of $N$ in $M$ is oriented and denote by $\tau_{N}^{M}$ the 
Thom class of $N$ in the cohomology group $H^{k}(M, M \backslash N ; \mathbb{Z})$, cf. [39, p. 118]. The image of $\tau_{N}^{M}$ by the restriction homomorphism $H^{k}(M, M \backslash N ; \mathbb{Z}) \rightarrow H^{k}(M ; \mathbb{Z})$, induced by the inclusion map $M \hookrightarrow(M ; M \backslash N)$, will be denoted by $[N]^{M}$ and called the cohomology class represented by $N$. If $M$ is compact and oriented, and $N$ is endowed with the compatible orientation, then $[N]^{M}$ is up to sign Poincaré dual to the homology class in $H_{*}(M ; \mathbb{Z})$ represented by $N$, cf. [39, p. 136].

Let $P$ be a smooth manifold and let $Q$ be a smooth submanifold of $P$. Let $f: M \rightarrow P$ be a smooth map transverse to $Q$. If the normal bundle of $Q$ in $P$ is oriented and the normal bundle of the smooth submanifold $N:=f^{-1}(Q)$ of $M$ is endowed with the orientation induced by $f$, then $\tau_{N}^{M}=f^{*}\left(\tau_{Q}^{P}\right)$, where $f$ is regarded as a map from $(M, M \backslash N)$ into $(P, P \backslash Q)$ (this follows form [28, p. 117, Theorem 6.7]). In particular, $[N]^{M}=f^{*}\left([Q]^{P}\right)$.

Let $\xi$ be an oriented smooth $\mathbb{R}$-vector bundle of rank $k$ on $M$. If $s: M \rightarrow \xi$ is a smooth section transverse to the zero section, and the normal bundle of $Z(s)=\{x \in M \mid s(x)=0\}$ is endowed with the orientation induced by $s$ from the orientation of $\xi$ (cf. the proof of Lemma 6.1), then

$$
e(\xi)=[Z(s)]^{M},
$$

where $e(\xi)$ stands for the Euler class of $\xi$. Indeed, let $E$ be the total space of $\xi$ and $p: E \rightarrow M$ the bundle projection. Identify $M$ with the image of the zero section of $\xi$. The section $s$ is transverse to $M$ and $Z(s)=s^{-1}(M)$. Consequently, $[Z(s)]^{M}=s^{*}\left([M]^{E}\right)$. Hence

$$
p^{*}\left([Z(s)]^{M}\right)=p^{*}\left(s^{*}\left([M]^{E}\right)\right)=(s \circ p)^{*}\left([M]^{E}\right)=[M]^{E},
$$

where the last equality holds since $s \circ p: E \rightarrow E$ is homotopic to the identity map. On the other hand, $p^{*}(e(\xi))=[M]^{E}$, cf. [39, p. 98]. It follows that $e(\xi)=[Z(s)]^{M}$ since $p^{*}$ is an isomorphism.

If $\xi$ is a $\mathbb{C}$-vector bundle of rank $k$, then

$$
e\left(\xi_{\mathbb{R}}\right)=c_{k}(\xi)
$$

where $\xi_{\mathbb{R}}$ is endowed with the orientation determined by the complex structure of $\xi$, cf. [39, p. 158].

Lemma 8.20. Let $M$ be a smooth manifold, and let $N$ be a smooth codimension 2 submanifold of $M$ with oriented normal bundle. Let $\lambda$ be a smooth $\mathbb{C}$-line bundle on $M$ with $c_{1}(\lambda)=[N]^{M}$. Then there exists a smooth section $s: M \rightarrow \lambda$ transverse to the zero section and satisfying $Z(s)=N$.

Proof. Let $\tau=(T, \rho, N)$ be a tubular neighborhood of $N$ in $M$. The smooth section $v: T \rightarrow \rho^{*} \tau$, defined by $v(x)=(x, x)$ for all $x$ in $T$, is transverse to the zero section and satisfies $Z(v)=N$. The differential of $v$ induces an isomorphism between the normal bundle to $N$ in $M$ and $\left.\left(\rho^{*} \tau\right)\right|_{N} \cong \tau$ (cf. the proof of Lemma 6.1). Via this isomorphism, $\tau$ is endowed with an orientation. Actually, $\tau$ can be regarded as a smooth $\mathbb{C}$-line bundle, being oriented of rank 2 (recall that $S O(2) \cong U(1)$ ). The restriction of $\rho^{*} \tau$ to $T \backslash N$ is a trivial smooth $\mathbb{C}$-line bundle. Consequently, the $\mathbb{C}$-line bundle $\rho^{*} \tau$ on $T$ and the standard trivial $\mathbb{C}$-line bundle $\varepsilon$ on $M \backslash N$ can be glued over $T \backslash N$. The resulting smooth $\mathbb{C}$-line bundle $\mu$ on $M$ has a smooth section $w: M \rightarrow \mu$, obtained by gluing $v$ and a nowhere zero section of $\varepsilon$, which is transverse to the zero section and satisfies $Z(w)=N$. It follows from the facts recalled before Lemma 8.20 that $c_{1}(\mu)=[N]^{M}$. The proof is complete since the smooth $\mathbb{C}$-line bundles $\lambda$ and $\mu$ are isomorphic. 
We now return to real algebraic geometry. Let $X$ be a compact nonsingular real algebraic variety. We say that a cohomology class $u$ in $H^{2}(X ; \mathbb{Z})$ is adapted if it is of the form $u=[Z]^{X}$, where $Z$ is a nonsingular Zariski locally closed subvariety of $X$ of codimension 2, which is closed in the Euclidean topology and whose normal bundle in $X$ is oriented.

Recall that the definition of an adapted smooth vector bundle is given in Section 1 . and then extended to topological vector bundles in Section 6 (before Theorem 6.9).

Proposition 8.21. For a topological $\mathbb{C}$-line bundle $\xi$ on a compact nonsingular real algebraic variety, the following conditions are equivalent:

(a) The $\mathbb{C}$-line bundle $\xi$ is adapted.

(b) The cohomology class $c_{1}(\xi)$ is adapted.

Proof. We may assume that the $\mathbb{C}$-line bundle $\xi$ is smooth. It follows form the definition of an adapted $\mathbb{C}$-line bundle that (a) implies (b). If (b) holds, then $\xi$ is adapted in view of Lemma 8.20.

Denote by $G(X)$ the subgroup of $H^{2}(X ; \mathbb{Z})$ generated by all adapted cohomology classes.

Theorem 8.22. For any compact nonsingular real algebraic variety $X$,

$$
G(X) \subseteq H_{\mathbb{C}-s t r}^{2}(X ; \mathbb{Z})
$$

In particular, if $\xi$ is a topological $\mathbb{C}$-line bundle on $X$ with $c_{1}(\xi)$ in $G(X)$, then $\xi$ admits a stratified-algebraic structure.

Proof. The inclusion $G(X) \subseteq H_{\mathbb{C} \text {-str }}^{2}(X ; \mathbb{Z})$ follows form Theorem 1.8 (with $\mathbb{F}=\mathbb{C}$ ) and Propositions 8.6 and 8.21 . Hence, the second assertion is a consequence of Proposition 8.6.

Theorem 8.22 is of interest since the group $G(X)$ is easier to compute directly than the group $H_{\mathbb{C} \text {-str }}^{2}(X ; \mathbb{Z})$. Adapted cohomology classes can also be used to give a complete description of $H_{\mathbb{C} \text {-str }}^{2}(X ; \mathbb{Z})$.

Theorem 8.23. Let $X$ be a compact real algebraic variety. For a cohomology class $u$ in $H^{2}(X ; \mathbb{Z})$, the following conditions are equivalent:

(a) The cohomology class $u$ belongs to $H_{\mathbb{C}-s t r}^{2}(X ; \mathbb{Z})$.

(b) There exists a filtration $\mathcal{F}=\left(X_{-1}, X_{0}, \ldots, X_{m}\right)$ of $X$ with $\overline{\mathcal{F}}$ a nonsingular stratification, and for each $i=0, \ldots, m$, there exists a multiblowup $\pi_{i}: X_{i}^{\prime} \rightarrow X_{i}$ over $X_{i-1}$ such that $X_{i}^{\prime}$ is a nonsingular variety and the cohomology class $\pi_{i}^{*}\left(\left.u\right|_{X_{i}}\right)$ in $H^{2}\left(X_{i}^{\prime} ; \mathbb{Z}\right)$ is adapted. Here $\left.u\right|_{X_{i}}$ is the image of $u$ under the homomorphism $H^{2}(X ; \mathbb{Z}) \rightarrow H^{2}\left(X_{i} ; \mathbb{Z}\right)$ induced by the inclusion map $X_{i} \hookrightarrow X$.

Proof. Let $\mathcal{F}=\left(X_{-1}, X_{0}, \ldots, X_{m}\right)$ be a filtration of $X$ with $\overline{\mathcal{F}}$ a nonsingular stratification, and for each $i=0, \ldots, m$, let $\pi_{i}: X_{i}^{\prime} \rightarrow X_{i}$ be a multiblowup over $X_{i-1}$ such that $X_{i}^{\prime}$ is a nonsingular variety. If $\xi$ is a topological $\mathbb{C}$-line bundle on $X$ with $c_{1}(\xi)=u$, then $c_{1}\left(\left.\xi\right|_{X_{i}}\right)=\left.u\right|_{X_{i}}$ and $c_{1}\left(\pi_{i}^{*}\left(\left.\xi\right|_{X_{i}}\right)\right)=\pi_{i}^{*}\left(\left.u\right|_{X_{i}}\right)$ for $0 \leq i \leq m$. In view of Proposition 8.21, the $\mathbb{C}$-line bundle $\pi_{i}^{*}\left(\left.\xi\right|_{X_{i}}\right)$ on $X_{i}^{\prime}$ is adapted if and only if the cohomology class $\pi_{i}^{*}\left(\left.u\right|_{X_{i}}\right)$ is adapted. Consequently, it suffices to combine Theorem 6.9 (with $\mathbb{F}=\mathbb{C}$ ) and Proposition 8.6 


\section{References}

[1] S. Akbulut and H. King, Submanifolds and homology of nonsingular real algebraic varieties, Amer. J. Math. 107 (1985), 45-83.

[2] S. Akbulut and H. King, Topology of Real Algebraic Sets, Mathematical Sciences Research Institute Publications, 25, Springer, 1992.

[3] M.F. Atiyah, K-Theory, W.A. Benjamin, 1967.

[4] M.F. Atiyah and F. Hirzebruch, Vector bundles and homogeneous spaces, Proc. Symp. in Pure Math., Amer. Math. Soc. 3 (1961), 7-38.

[5] R. Benedetti and A. Tognoli, On real algebraic vector bundles, Bull. Sci. Math. (2) 104 (1980), 89-112.

[6] R. Benedetti and A. Tognoli, Remarks and counterexamples in the theory of real algebraic vector bundles and cycles, Géométrie algébrique réelle et formes quadratiques. Lecture Notes in Math. 959, 198-211, Springer, 1982.

[7] M. Bilski, W. Kucharz, A. Valette and G. Valette, Vector bundles and regulous maps, Math. Zeitschrift, to appear.

[8] J. Bochnak, M. Buchner and W. Kucharz, Vector bundles over real algebraic varieties, K-Theory 3 (1989), 271-298. Erratum: K-Theory 4 (1990), 103.

[9] J. Bochnak, M. Coste and M.-F. Roy, Real Algebraic Geometry, Ergeb. der Math. und ihrer Grenzgeb. Folge 3, vol. 36, Springer, 1998.

[10] J. Bochnak and W. Kucharz, Algebraic approximation of mappings into spheres, Michigan Math. J. 34 (1987), 119-125.

[11] J. Bochnak and W. Kucharz, Realization of homotopy classes by algebraic mappings, J. Reine Angew. Math. 377 (1987), 159-169.

[12] J. Bochnak and W. Kucharz, On real algebraic morphisms into even-dimensional spheres, Ann. of Math. (3) 128 (1988), 415-433.

[13] J. Bochnak and W. Kucharz, K-theory of real algebraic surfaces and threefolds, Math. Proc. Cambridge Philos. Soc. 106 (1989), 471-480.

[14] J. Bochnak and W. Kucharz, Vector bundles on a product of real cubic curves, K-Theory 6 (1992), 487-497.

[15] J. Bochnak and W. Kucharz, The homotopy groups of some spaces of real algebraic morphisms, Bull. London Math. Soc. 25 (1993), 385-392.

[16] J. Bochnak and W. Kucharz, Elliptic curves and real algebraic morphisms, J. Algebraic Geometry 2 (1993), 635-666.

[17] J. Bochnak and W. Kucharz, On homology classes represented by real algebraic varieties, Singularities Symposium - Łojasiewicz 70, Banach Center Publ. Vol. 44, 21-35, Polish Acad. Sci., Warsaw, 1998. 
[18] J. Bochnak, W. Kucharz and R. Silhol, Morphisms, line bundles and moduli spaces in real algebraic geometry, Inst. Hautes Études Sci. Publ. Math. 86 (1997), 5-65.

[19] J. Bochnak and W. Kucharz, Real algebraic morphisms represent few homotopy classes, Math. Ann. 337 (2007), 909-921.

[20] A. Borel et A. Haefliger, La classe d'homologie fondamentale d'un espace analytique, Bull. Soc. Math. France 89 (1961), 461-513.

[21] Th. Bröcker and K. Janich, Introduction to Differential Topology, Cambridge University Press, 1982.

[22] E. Dyer, Cohomology Theories, Benjamin, 1969.

[23] G. Fichou, J. Huisman, F. Mangolte and J.-Ph. Monnier, Fonctions régulues, arXiv:1112.3800v2 [math.AG], 2012.

[24] W. Fulton, Intersection Theory, Ergeb. der Math. und ihrer Grenzgeb. Folge 3, vol. 2, Springer, 1984.

[25] R. Ghiloni, Second order homological obstructions on real algebraic manifolds, Topology Appl. 154 (2007), 3090-3094.

[26] P. Griffiths and J. Adams, Topics in Algebraic and Analytic Geometry, Mathematical Notes 13, Princeton University Press, Princeton, NJ, 1974.

[27] H. Hironaka, Resolution of singularities of an algebraic variety over a field of characteristic zero, Ann. of Math. 79 (1964), 109-326.

[28] M. Hirsch, Differential Topology, Springer, 1976.

[29] D. Husemoller, Fibre Bundles, Springer, 1975.

[30] M. Karoubi, K-Theory, Grundlehren der Mathematischen Wissenschaften 226, Springer, 1978.

[31] J. Kollár, Lectures on Resolution of Singularities, Ann. of Math. Studies, vol. 166, Princeton University Press, Princeton, NJ, 2007.

[32] J. Kollár and K. Nowak, Continuous rational functions on real and $p$-adic varieties, arXiv:1301.5048v1 [math.AG], 2013.

[33] W. Kucharz, Rational maps in real algebraic geometry, Adv. Geom. 9 (2009), 517539.

[34] W. Kucharz, Complex cycles on algebraic models of smooth manifolds, Math. Ann. 346 (2010), 829-856.

[35] W. Kucharz, Approximation by continuous rational maps into spheres, J. Eur. Math. Soc. (JEMS), to appear.

[36] K. Kurdyka, Ensemble semi-algébriques symétriques par arcs, Math. Ann. 282 (1988), 445-462. 
[37] K. Kurdyka, Injective endomorphisms of real algebraic sets are surjective, Math. Ann. 313 (1999), 69-82.

[38] K. Kurdyka and A. Parusiński, Arc-symmetric sets and arc-analytic mappings, Arc spaces and additive invariants in real algebraic and analytic geometry, 33-67, Panor. Synthèses 24, Soc. Math. France, Paris, 2007.

[39] J.W. Milnor and J.D. Stasheff, Characteristic Classes, Ann. of Math. Studies 76, Princeton University Press, Princeton, NJ, 1974.

[40] A. Parusiński, Topology of injective endomorphisms of real algebraic sets, Math. Ann. 328 (2004), 353-372.

[41] F.P. Peterson, Some remarks on Chern classes, Ann. of Math. 69 (1958), 414-420.

[42] J.-P. Serre, Faisceaux algébriques coherents, Ann. of Math. (2) 61 (1955), 197-278.

[43] E. Spanier, Algebraic Topology, Springer, 1966.

[44] R.G. Swan, Vector bundles and projective modules, Trans. Amer. Math. Soc. 105 (1962), 264-277.

[45] R.G. Swan, Topological examples of projective modules, Trans. Amer. Math. Soc. 230 (1977), 201-234.

Wojciech Kucharz

Institute of Mathematics

Faculty of Mathematics and Computer Science

Jagiellonian University

ul. Łojasiewicza 6

30-348 Kraków

Poland

e-mail: Wojciech.Kucharz@im.uj.edu.pl

Krzysztof Kurdyka

Laboratoire de Mathématiques

UMR 5175 du CNRS

Université de Savoie

Campus Scientifique

73376 Le Bourget-du-Lac Cedex

France

e-mail: kurdyka@univ-savoie.fr 